Aus der Abteilung Pädiatrie II- Neuropädiatrie

(Prof. Dr. med. J. Gärtner)

im Zentrum Kinderheilkunde und Jugendmedizin

der Medizinischen Fakultät der Universität Göttingen

\title{
Adipositas im Kindes- und Jugendalter: Charakterisierung eines \\ Therapiekollektivs und Prädiktoren für Therapieerfolg
}

\author{
Inaugural-Dissertation \\ zur Erlangung des Doktorgrades \\ der Medizinischen Fakultät \\ der Georg-August-Universität zu Göttingen \\ vorgelegt von \\ Tatjana Tamara König \\ aus \\ Hannover
}


Dekan:

I. Berichterstatterin:

II. Berichterstatter:

III. Berichterstatter:
Prof. Dr. med. C. Frömmel

Prof. Dr. med. J. Gärtner

Priv.-Doz. Dr. med. T. Ellrott

Prof. Dr. med., Dr. rer. nat. T. Crozier

Tag der mündlichen Prüfung: 15.02.2012 
Abkürzungsverzeichnis

$\begin{array}{ll}\text { ACTH } & \text { Adrenocorticotropes Hormon } \\ \text { AGA } & \text { Arbeitsgemeinschaft Adipositas im Kindes- und Jugendalter } \\ \text { AHA } & \text { American Heart Association } \\ \text { APV } & \text { Adipositas-Patienten-Verlaufs(-Programm) } \\ \text { AR } & \text { Adipositas Rebound } \\ \text { aMSH } & \text { Alpha Melanozyten Stimulierendes Hormon } \\ \text { BMI } & \text { Body-Mass-Index } \\ \text { BMI-SDS } & \text { Body-Mass-Index-Standard-Deviation-Score } \\ \text { C-PAP } & \text { Continuous positive Airway Pressure } \\ \text { CT } & \text { Computertomographie } \\ \text { DBP } & \text { Diastolic Blood Pressure } \\ \text { DEXA } & \text { Dual Energy X-Ray Absorptiometry } \\ \text { EV } & \text { Erstvorstellung } \\ \text { GH } & \text { Growth Hormone } \\ \text { HDL } & \text { High Density Lipoprotein } \\ \text { HOMA-IR } & \text { Homeostasis Model Assessment of Insulin Resistance } \\ \text { IDF } & \text { International Diebetes Federation } \\ \text { IRS } & \text { Insulin Resistance Syndrome } \\ \text { IOTF } & \text { International Obesity Taskforce } \\ \text { KiGGS } & \text { Kinder- und Jugendgesundheitssurvey: bundesweite Studie des Robert Koch- } \\ & \text { Instituts (RKI) in Zusammenarbeit mit dem Ministerium für Gesundheit (BMG) } \\ & \text { und dem Ministerium für Bildung und Forschung (BMBF) } \\ \text { LDL } & \text { Low Density Lipoprotein } \\ \text { LMS } & \text { Akronym der Variablen der LMS-Methode [lambda, my, sigma] } \\ \text { Lp(a) } & \text { Lipoprotein(a) } \\ \text { MAD } & \text { mittlerer arterieller Druck } \\ \text { MRT } & \text { Magnet-Resonanz-Tomographie } \\ \text { MSH } & \text { Melanozyten Stimulierendes Hormon } \\ \text { NAFLK } & \text { nicht-alkoholische Fettlebererkrankung } \\ \text { NHBPEP } & \text { National High Blood Pressure Education Program } \\ \text { OGTT } & \text { oraler Glukosetoleranztest } \\ \text { PCOS } & \text { Polycystic Ovary Syndrome } \\ \text { POMC } & \text { Proopiomelanocortin } \\ \text { Ref } & \text { Referenzgruppe } \\ \text { SAS } & \text { Schlaf-Apnoe-Syndrom } \\ \text { SBP } & \text { Systolic Blood Pressure } \\ \text { SHBG } & \text { Sexualhormon bindendes Globulin } \\ \text { SPZ } & \text { Sozialpädiatrisches Zentrum } \\ \text { SSW } & \text { Schwangerschaftswoche } \\ \text { T2DM } & \text { Typ-2-Diabetes mellitus } \\ \text { TG } & \text { Triglyceride } \\ & \end{array}$ 


\section{Inhalt}

1. Einleitung _ 11

1.1. Definition _ 11

1.2. Epidemiologie __ 12

1.3. Ursachen der Adipositas ___ 14

1.3.1. Sekundäre Adipositas___ 14

1.3.1.1. Genetische Ursachen ___ 14

1.3.1.1.1. Multigener Vererbungsmodus___ 14

1.3.1.1.2. Monogener Vererbungsmodus _ 15

1.3.1.1.3. Adipositas-Syndrome __ 16

1.3.1.2. Psychiatrische Grunderkrankungen __ 17

1.3.1.3. Endokrinologische Grunderkrankungen ___ 18

1.3.1.3.1. Hypothyreose _ 18

1.3.1.3.2. Wachstumshormonmangel___ 18

1.3.1.3.3. Hyperkortisolismus ___ 18

1.3.1.4. Andere Erkrankungen___ 19

1.3.1.5. Medikamenteninduzierte Adipositas __ 20

1.3.2. Primäre Ursachen: Lifestyle __ 20

1.3.2.1. Ernährung__ 20

1.3.2.2. Körperliche Aktivität ___ 23

1.3.2.3. Weitere Einflussfaktoren___ 25

1.3.2.3.1. Familie _ 25

1.3.2.3.2. Perinatale Einflüsse __ 26

1.3.2.3.3. Adipositas- Rebound __ 27

1.3.3. Risikogruppen__ 28

1.3.3.1. Sozialstatus _28

1.3.3.2. Migrationshintergrund___ 29

1.3.3.3. Intellektuelle Fähigkeiten __ 29

1.4. Folgeerkrankungen _ 30

1.4.1. Das Metabolische Syndrom ___ 30

1.4.1.1. Störungen der Glukosetoleranz __ 31

1.4.1.2. Steatosis hepatis _ 31

1.4.1.3. Dyslipoproteinämie ___ 32

1.4.1.4. Arterielle Hypertonie __ 32

1.4.2. Hyperurikämie___ 35

1.4.3. Weitere kardiovaskuläre Risikofaktoren ___ 36

1.4.3.1. Lipoprotein(a) _ 36 
1.4.3.2. Hyperhomozysteinämie 36

1.4.4. Syndrom der polyzystischen Ovarien (PCOS) __ 37

1.4.5. Störungen der Pubertätsentwicklung ___ 38

1.4.6. Adipositas-assoziierte Erkrankungen des Bewegungsapparates___ 38

1.4.6.1. Wirbelsäule ___ 38

1.4.6.2. Beinachse___ 39

1.4.6.3. Fußskelett___ 39

1.4.6.4. Epiphysiolysis capitis femoris __ 40

1.4.6.5. Blount Disease ___ 40

1.4.6.6. Frakturen und Traumata___ 41

1.4.7. Schlaf-Apnoe-Syndrom___ 41

1.4.8. Psychiatrische Co-Morbiditäten _ 42

1.5. Fragestellung___ 43

2. Material/Methoden__ 44

2.1. Datenerfassung und -auswertung ___ 44

2.2. Status bei Erstvorstellung ___ 44

2.2.1. Biometrische Daten __ 44

2.2.2. Anamnese___ 45

2.2.3. Begleiterkrankungen___ 46

2.2.3.1. Metabolisches Syndrom___ 46

2.2.3.1.1. Insulinresistenz und Störungen der Glukosetoleranz ___ 46

2.2.3.1.2. Steatosis hepatis __ 47

2.2.3.1.3. Dyslipoproteinämie und Triglyceridämie___ 47

2.2.3.1.4. Arterielle Hypertonie ___ 48

2.2.3.1.5. Übersicht über die Komponenten des metabolischen Syndroms___ 48

2.2.3.2. Leptin _ 49

2.2.3.3. Hyperurikämie __ 49

2.2.3.4. Kardiovaskuläre Risikofaktoren __ 49

2.2.3.4.1. Lipoprotein(a) _ 49

2.2.3.4.2. Hyperhomozysteinämie __ 49

2.3. Verlauf der Gewichtsentwicklung vor der Intervention __ 49

2.3.1. Analyse der Daten aus den Vorsorgeuntersuchungen___ 49

2.3.2. Regression des Gewichtsverlaufes vor der Intervention __ 50

2.4. Therapiegruppen__ 50

2.4.1. Beurteilung des Therapieerfolges___ 50 
3. Ergebnis 52

3.1. Status bei Erstvorstellung (EV) __ 52

3.1.1. Biometrische Daten __ 52

3.1.2. Psychosoziale Faktoren ___ 54

3.1.2.1. Charakterisierung der Familien__ 54

3.1.2.2. Migrationshintergrund___ 55

3.1.2.3. BMI der Eltern___ 55

3.1.2.4. Ausbildung und Berufstätigkeit der Eltern___ 57

3.1.2.5. Schulbildung der Kinder und Jugendlichen ___ 58

3.2. Folge- und Begleiterkrankungen __ 60

3.2.1. Metabolisches Syndrom __ 60

3.2.1.1. HOMA-IR als Zeichen der Insulinresistenz___ 60

3.2.1.2. Störungen der Glukosetoleranz __ 62

3.2.1.3. Steatosis hepatis ___ 63

3.2.1.4. Fettstoffwechsel __ 64

3.2.1.4.1. Triglyceride __ 64

3.2.1.4.2. Lipoproteine __ 65

3.2.1.5. Arterielle Hypertonie ___ 65

3.2.1.6. Übersicht der Komponenten des metabolischen Syndroms___ 66

3.2.2. Hyperurikämie___ 68

3.2.3. Leptin _ 69

3.2.4. Weitere kardiovaskuläre Risokofaktoren __ 70

3.2.4.1. Lipoprotein(a) _ 70

3.2.4.2. Hyperhomozysteinämie __ 70

3.2.4.3. Übersicht der zusätzlichen kardiovaskulären Risikofaktoren ___ 71

3.3. Gewichtsverlauf vor der Intervention __ 71

3.3.1. Analyse der Daten aus den Vorsorgeuntersuchungen___ 71

3.3.2. Regression des Gewichtsverlaufs vor der Intervention ___ 73

3.3.3. Zusammenhang mit Geburtsgewicht und Geburtswoche ___ 74

3.4. Erfolg der Intervention _ 75

3.4.1. Therapiegruppen

3.4.2. Psychosoziale Faktoren ___ 80

3.4.2.1. Migrationshintergrund___ 80

3.4.2.2. Ausbildung und Berufstätigkeit der Eltern___ 80

3.4.2.3. Weiterführende Schulbildung der Kinder und Jugendlichen___ 81 
4.1. Status bei Erstvorstellung ___ 83

4.1.1. Biometrische Daten ___ 83

4.1.2. Psychosoziale Faktoren ___ 84

4.1.2.1. Charakterisierung der Familien___ 84

4.1.2.2. Migrationshintergrund___ 84

4.1.2.3. BMI der Eltern ___ 85

4.1.2.4. Ausbildung und Berufstätigkeit der Eltern___ 86

4.1.2.5. Schulbildung der Kinder und Jugendlichen ___ 86

4.1.3. Begleiterkrankungen___ 87

4.1.3.1. Metabolisches Syndrom ___ 87

4.1.3.1.1. Insulinresistenz und Störungen der Glukosetoleranz ___ 87

4.1.3.1.2. Steatosis hepatis ___ 88

4.1.3.1.3. Fettstoffwechselstörungen __ 88

4.1.3.1.4. Arterielle Hypertonie ___ 89

4.1.3.1.5. Zusammenfassung zum metabolischen Syndrom ___ 89

4.1.3.2. Hyperurikämie___ 91

4.1.3.3. Weitere kardiovaskuläre Risikofaktoren ___ 91

4.1.3.3.1. Lipoprotein(a) __ 91

4.1.3.3.2. Hyperhomozytseinämie ___ 91

4.1.3.3.3. Übersicht über die zusätzlichen kardiovaskulären Risikofaktoren ___ 91

4.1.3.4. Leptin __ 92

4.2. Verlauf vor der Intervention___ 92

4.2.1. Analyse der Daten aus den Vorsorgeuntersuchungen___ 92

4.2.2. Regression des Gewichtsverlaufes vor der Intervention ___ 93

4.2.3. Zusammenhang mit Geburtsgewicht und Geburtswoche ___ 93

4.3. Therapieerfolg __ 94

4.3.1. Therapiegruppen __ 95

4.3.2. Psychosoziale Faktoren ___ 96

4.3.2.1. Migrationshintergrund___ 96

4.3.2.2. Ausbildung und Berufstätigkeit der Eltern___ 96

4.3.2.3. Weiterführende Schulbildung der Kinder und Jugendlichen___ 96

5. Zusammenfassung__ 97

6. Literaturverzeichnis 


\section{Tabellenverzeichnis}

Tabelle Nr. Seite

1: Adipositas-Definition bei Erwachsenen _ـ 11

2: Symptome seltener Adipogener Syndrome __ 17

3: Psychiatrische Ursachen für Adipositas___ 17

4: Verzehr unterschiedlicher Lebensmittelgruppen _ـ 22

5: Definition des metabolischen Syndroms _ 30

6: Richtwerte für die Manschettengröße zur Blutdruckmessung bei Kindern und Jugendlichen__ 33

7: Klassifizierung der arteriellen Hypertonie im Kindes- und Jugendalter ___ 34

8: Ursachen einer sekundären arteriellen Hypertonie im Kindes- und Jugendalter _ 34

9: Mögliche Ursachen einer erhöhten Homozystein-Serum-Konzentration ___ 37

10: Gemeinsame Co-Morbiditäten von SAS und Adipositas ___ 42

11: HOMA-IR Referenzwerte ___ 46

12: Methoden zur Bestimmung der Serumlipide __ 47

13: Definition der arteriellen Hypertonie beim Kind in der Langzeitblutdruckmessung 48

14: Festlegung der Kriterien zur Definition des metabolischen Syndroms___ 48

15: Einteilung der Therapiegruppen im SPZ Göttingen __ 50

16: Gewichtsverteilung der Eltern__ 55

17: Gemeinsame Betrachtug des BMI beider Elternteile ___ 56

18: Korrelation des BMI-SDS von Eltern und Kindern in der linearen Regression des BMI-SDS 56

19: Schulbildung der Kinder des Kollektivs __ 58

20: Insulinresistenz in \% nach Alter und Geschlecht __ 60

21: Triglyceride bei Erstvorstellung nach Alter und Geschlecht___ 64

22: Prozentuale Lp(a)-Erhöhung im Kollektiv bei Erstvorstellung __ 70

23: Zusammenhang zwischen Adipositas, Geburtswoche und Geburtsgewicht ___ 74

24: Mittelwerte der Reduktion des BMI-SDS nach sechs und zwölf Monaten für Jungen und Mädchen

25: Verteilung der Patienten in den Therapiegruppen und durchschnittliche Reduktion des BMI-SDS nach sechs und zwölf Monaten

26: Durchschnittliche Differenz des BMI-SDS nach weiterführender Schule nach sechs und zwölf Monaten

27: Vergleich des Anteils kinderreicher Familien im Kollektiv und in Gesamtdeutschland 84

28: Übersicht zur Inzidenz von Begleiterkrankungen im Kollektiv 90 


\section{Abbildungsverzeichnis}

Abbildung $\mathrm{Nr}$. Seite

1: Körperfettanteil und Alter 11

2: Prävalenz von Übergewicht unter Schulkindern in Europa 12

3: "Neue Perzentile“: Die Perzentile der KIGGS-Studie im Vergleich zu denen nach Kromeyer-Hauschild 13

4: $\quad$ Multigene Vererbung von Adipositas 14

5: Medizinische Ursachen für Adipositas 19

6: Die aid-Ernährungs-Pyramide 21

7: Anteil der Kinder, die täglich frisches Obst essen 21

8: Anteil der Kinder, die täglich gekochtes Gemüse Tiefkühlgemüse, Konservengemüse und Blattsalat, Rohkost oder Rohes Gemüse konsumieren 21

9: Zusammenhang zwischen körperlicher Inaktivität und PC-/Fernsehkonsum in der KiGGS-Studie 24

10: Zusammenhang zwischen Adipositas und PC-/ Fernsehkonsum in der KiGGS-Studie 25

11: Prävalenz von Adipositas nach Alter der Kinder und BMI der Mutter in der KiGGS-Studie 25

12: Prävalenz von Adipositas nach sozialem Status in der KiGGS- Studie 28

13: Prävalenz von Adipositas nach Migrationshintergrund in der KiGGS- Studie 29

14: Acanthosis nigricans im Nacken 31

15: Zusammenhang zwischen Insulinresistenz und PCOS 37

16: Verteilung des BMI bei Erstvorstellung 52

17: Alter bei Erstvorstellung 53

18: BMI-SDS nach Geschlecht und Altersgruppen bei Erstvorstellung 53

19: BMI-SDS bei Erstvorstellung nach Anzahl der Geschwister 54

20: BMI-SDS bei Erstvorstellung nach BMI der Eltern 56

21: BMI-SDS bei Erstvorstellung nach Schulbildung der Eltern 57

22: BMI-SDS bei Erstvorstellung nach Berufstätigkeit der Eltern 58

23: BMI-SDS nach weiterführender Schulbildung der Kinder 59

24: HOMA-Werte und BMI-SDS der Jungen 61

25: HOMA-Werte und BMI-SDS der Mädchen 61

26: Anteil auffälliger Werte im OGTT bei Nüchtern-Glukose und 2-Stunden-Wert 62

27: Transaminasen bei Erstvorstellung 63

28: Transaminasen und Durchschnittlicher BMI-SDS bei Erstvorstellung 63

29: Transaminasen und Durchschnittliches Alter bei Erstvorstellung 64

30: Cholesterin bei Erstvorstellung nach Alter und Geschlecht 65 
31: Anteil pathologischer 24-Stunden-Blutdruckmessungen 66

32: Prozentualer Anteil nach Anzahl der manifesten Folgeerkrankungen 67

33: Anzahl der Folgeerkrankungen und Alter bei Jungen und Mädchen 68

34: Anzahl der Folgeerkrankungen und BMI-SDS bei Jungen und Mädchen 68

35: Harnsäurespiegel bei Erstvorstellung nach Alter und Geschlecht 69

36: Korrelation von BMI-SDS und Leptinspiegel bei Mädchen und Jungen $69 / 70$

37: Anteil der Kinder mit zusätzlich zum metabolischen Syndrom erhöhtem kardiovaskulären Risiko 71

38: BMI-SDS-Verteilung bei den Vorsorgeuntersuchungen der Mädchen 72

39: BMI-SDS-Verteilung bei den Vorsorgeuntersuchungen der Jungen 72

40: Gewichtsverlauf der Mädchen vor der Intervention 73

41: Gewichtsverlauf der Jungen vor der Intervention 74

42: Reduktion des BMI-SDS nach sechs und zwölf Monaten 75

43: Erfolg nach sechs und zwölf Monaten im Vergleich 76

44: Altersverteilung nach Therapieerfolg 76

45: Differenz des BMI-SDS nach sechs und zwölf Monaten nach Altersgruppen 77

46: Differenz des BMI-SDS nach sechs und zwölf Monaten nach Geschlecht 78

47: Differenz im BMI-SDS nach Therapiegruppen nach sechs und zwölf Monaten 79

48: Differenz des BMI-SDS mit und ohne Migrationshintergrund nach sechs und zwölf Monaten 80

49: Differenz das BMI-SDS der Hauptschüler im Vergleich nach sechs und zwölf Monaten 81

50: BMI der Mütter des Kollektivs im gesamtdeutschen Vergleich 85 


\section{Einleitung}

\subsection{Definition}

Adipositas beschreibt den Zustand eines erhöhten Fettanteils an der Gesamtkörpermasse. Zur Bestimmung des Körperfettanteils stehen verschiedene Methoden zur Verfügung, beispielsweise mechanische Methoden, wie die Calipermetrie (Messung von Hautfaltendicke) oder die Messung des Taillenumfangs. Diese sind kostengünstig und wenig invasiv, aber auch schwierig zu standardisieren und daher ungenau.

Weitere mögliche Verfahren sind radiologische Methoden, wie die Bestimmung der Dicke der Fettschicht mittels Sonographie, MRT oder CT, Ganzkörper-DEXA (Dual Energy X-Ray Absorptiometry), Isotopenmethoden oder die bioelektrische Impedanzanalyse. Es handelt sich hierbei jedoch um aufwändige, kostspielige Untersuchungen, die daher im klinischen Alltag nicht praktikabel sind.

Internationaler Standard ist daher die Bestimmung des BMI aus BMI=Körpergewicht [kg]/ Körperlänge $[\mathrm{m}]^{2}$ als Messgröße, die ein genauer definierbares Maß darstellt als die

\begin{tabular}{|l|l|}
\hline BMI $\left[\mathrm{kg} / \mathrm{m}^{2}\right]$ & Bewertung \\
\hline$<18,5$ & Untergewicht \\
\hline $18,5-25$ & Normalgewicht \\
\hline $25-30$ & Übergewicht \\
\hline $30-35$ & Adipositas Grad I \\
\hline $35-40$ & Adipositas Grad II \\
\hline$>40$ & Adipositas Grad III \\
\hline
\end{tabular}
Calipermetrie oder die Messung des Taillenumfanges, gleichzeitig aber eine gut durchführbare Untersuchung als Grundlage hat [Schauder und Arends 2006]. Von der WHO wurden für Erwachsene die in Tabelle 1 dargestellten Grenzwerte festgelegt.

Tabelle 1: Adipositas-Definition bei Erwachsenen [Schauder und Arends 20 06, S. 486]

Der physiologische Körperfettanteil schwankt stark mit dem Alter und ab der Pubertät auch mit dem Geschlecht (Abb.1), deshalb werden

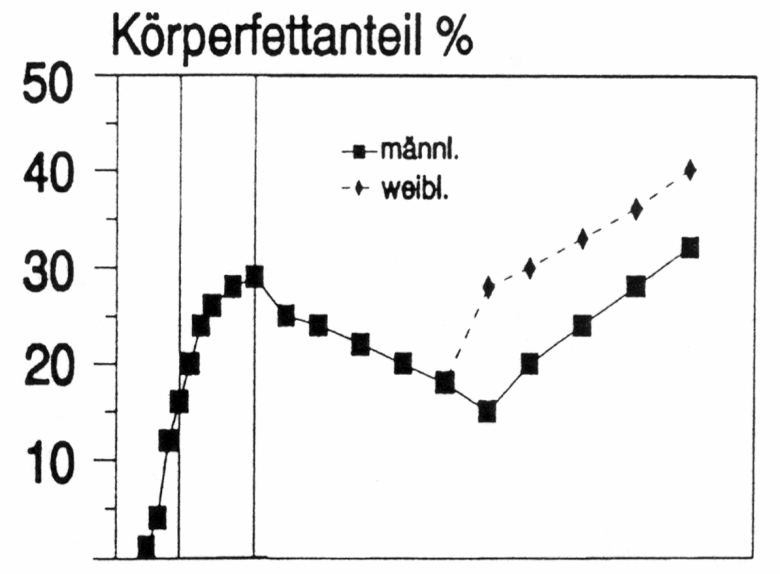

Goburt $1 \begin{array}{llllllllll}2 & 3 & 6 & 9 & 12 & 18 & 25 & 45 & 65 & 85\end{array}$ Alter (Jahre) für die Definition von Übergewicht, Adipositas und extremer Adipositas im Kindes- und Jugendalter in Deutschland die Body-MassIndex (BMI) Perzentile nach KromeyerHauschild et al. von der Arbeitsgemeinschaft Adipositas im Kindes- und Jugendalter (AGA) empfohlen. Kinder werden demnach oberhalb des 90 . Perzentils als übergewichtig, oberhalb des 97. Perzentils als adipös und oberhalb des 99,5. Perzentils als extrem adipös eingestuft [AGA 2006] 
Anhand des BMI lässt sich die zentrale Fettmasse jedoch nicht beurteilen. KromeyerHauschild et al. empfehlen daher die zusätzliche Bestimmung des Taillenumfanges, um Kinder mit einer erhöhten zentralen Fettmasse zu identifizieren. In einer Studie mit 2544 Kindern wurden hierfür Perzentile bestimmt [Kromeyer-Hauschild et al. et al. 2008].

Vor 2006 galten für Deutschland die Perzentile nach Rolland-Cachera von 1982. Die Messungen, die die Grundlage für diese Perzentilen bildeten, begannen bereits 1953 [Rolland-Cachera et al. 1982].

Im angloamerikanischen Sprachraum richtet man sich nach der Definition der International Obesity Taskforce (IOTF) [Cole et al. 2000]. Hier wird auch ein BMl oberhalb des 85. Perzentils als Übergewicht und oberhalb des 95. Perzentils als Adipositas klassifiziert. So lassen sich Studien international oft nur schwer vergleichen.

\subsection{Epidemiologie}

Adipositas bei Kindern und Jugendlichen nimmt bedrohliche Ausmaße an.

Olshansky et al. gelangten 2005 zu dem Ergebnis, dass, auf Grund der zunehmenden Adipositas in den USA, zum ersten Mal seit zwei Jahrhunderten die Lebenserwartung der jüngeren Generation die der älteren nicht übersteigen wird [Olshansky et al. 2005].

Die KiGGS-Studie [Kurth und Schaffrath-Rosario 2007] ergab, dass auch in Deutschland der Anteil übergewichtiger Kinder und Jugendlicher gegenüber den Achtziger- und

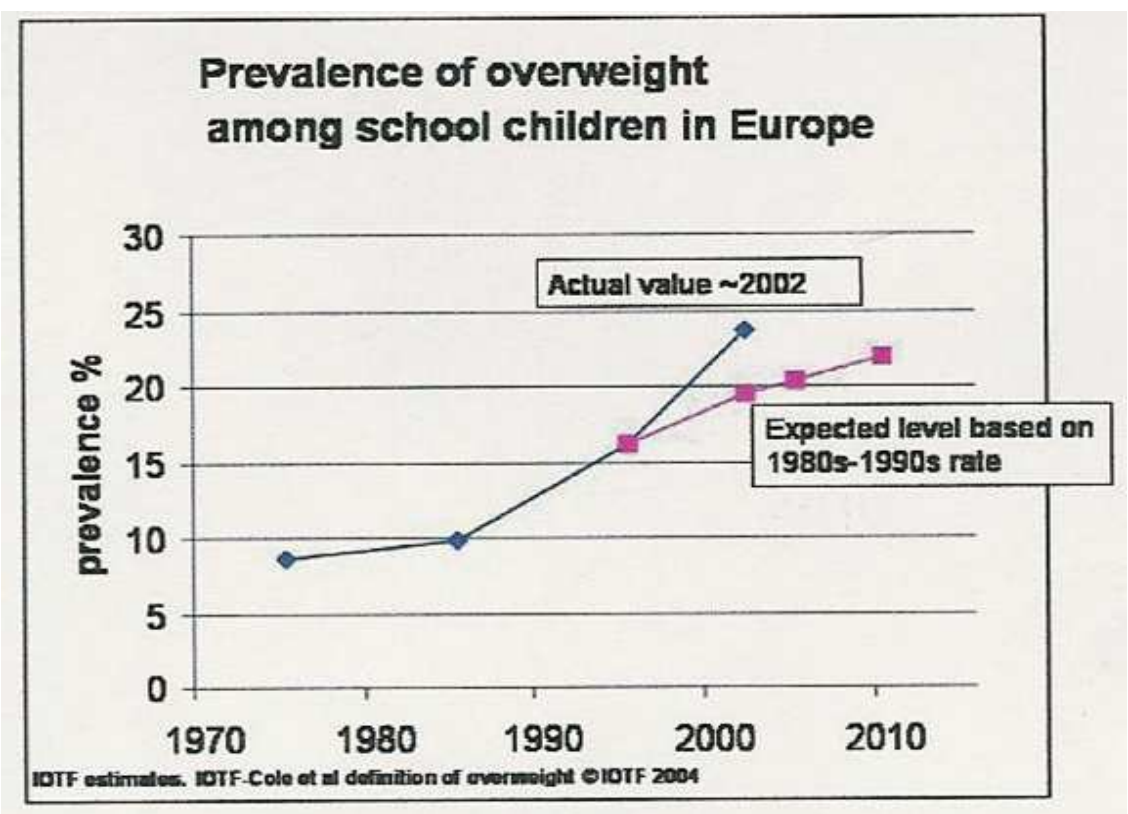

Neunzigerjahren um 50\% zugenommen hat. Betroffen sind vor allem die Jugendlichen. 15\% der Kinder und Jugendlichen im Alter von 3-17 Jahren sind übergewichtig, davon $6,3 \%$ adipös. In realen Zahlen entspricht das 1,9 Millionen Übergewichtiger und 800.000 Adipöser [Kurth und SchaffrathRosario 2007]. 
Die von der ITOF für 2010 prognostizierte Prävalenz von Adipositas bei europäischen Kindern wurde schon 2002 überschritten.

Im Vergleich dazu waren in den Vereinigten Staaten im Jahr 2007 31,6\% der 10-17-Jährigen von Übergewicht, 16,4\% von Adipositas betroffen [Singh et al. 2010].

Vergleicht man die Perzentile nach Kromeyer-Hauschild et al. (2008) von 2001 mit neuen Perzentilen von 2007, die aus den Ergebnissen der KiGGS-Studie (2003-2006) hervorgehen, wird die deutliche Zunahme des Übergewichts und der Adipositas in allen Altersgruppen in den letzten Jahren besonders deutlich [Kurth und Schaffrath-Rosario 2007].

Ein Vergleich mit den Perzentilen nach Rolland-Cachera et al. (1982) stellt sich dieser Unterschied noch gravierender dar.
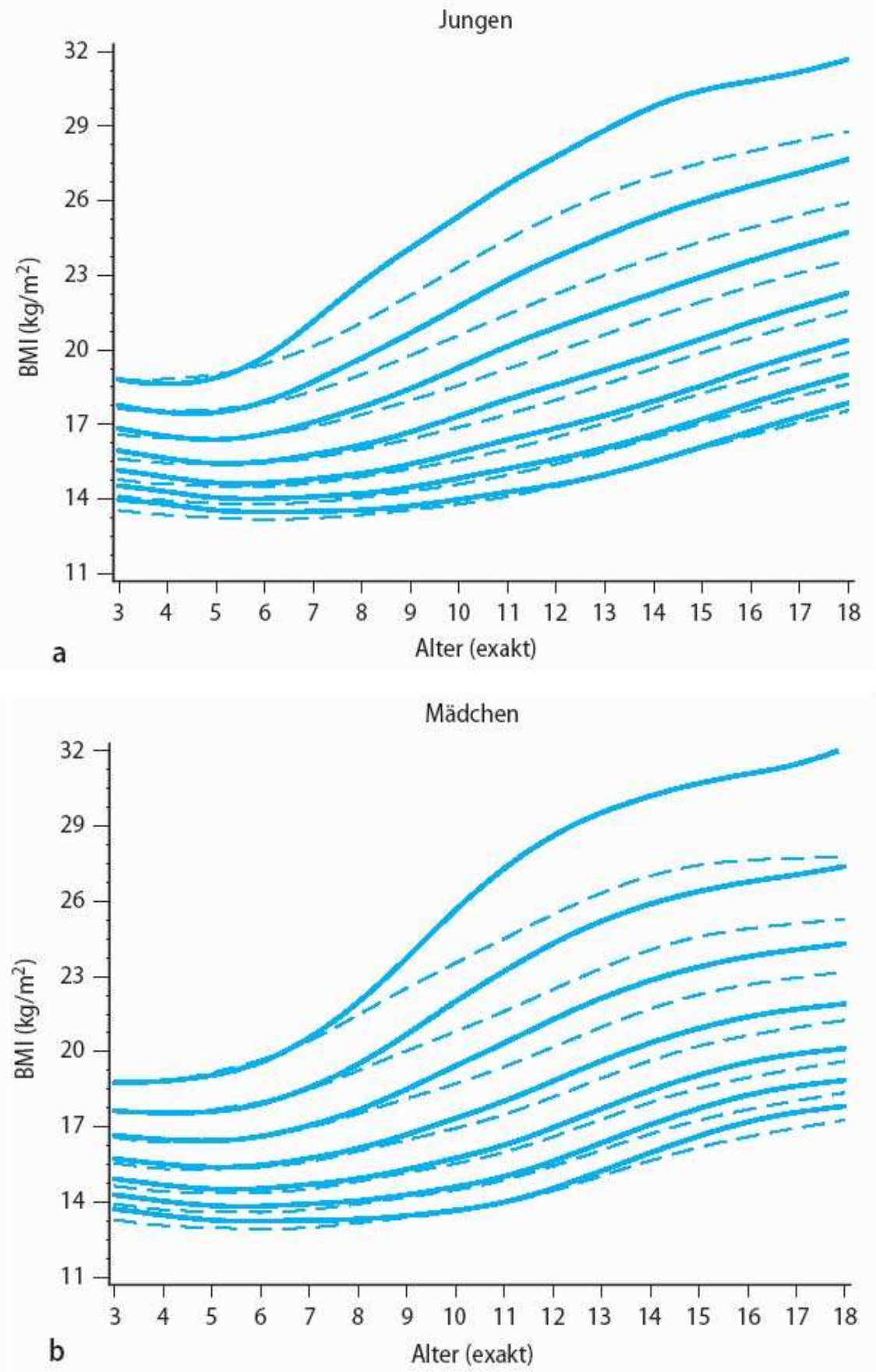

Abbildung 3a und $3 b$ :

"Neue Perzentile"

Die Perzentile der KIGGS-

Studie (durchgezogene

Linien) im Vergleich zu

den (gestrichelten)

Perzentilen nach

Kromeyer-Hauschild et al.

[Kurth und Schaffrath-

Rosario 2007, S.741] 


\subsection{Ursachen der Adipositas}

\subsubsection{Sekundäre Adipositas}

Eine sekundäre Adipositas, die ursächlich auf eine andere Grunderkrankung zurückführbar ist, liegt nur bei einem sehr geringen Anteil der Betroffenen vor. In diesem Abschnitt sollen mögliche Ursachen vorgestellt werden.

\subsubsection{Genetische Ursachen}

Eine häufige Laienätiologie ist die genetisch bedingte Entstehung von Adipositas. Unter den Müttern von Kindern, die in eine Spezialklinik für Adipöse aufgenommen wurden, gaben 30\% als vermutete Ursache aus den sechs Auswahlmöglichkeiten „Genetik, isst zu viel, Stress, träger Stoffwechsel, Trägheit und Lifestyle“ die „Genetik“ an [Watkins et al. 2007].

\subsection{Multigener Vererbungsmodus}

Man kann davon ausgehen, dass ein Teil der Bevölkerung eine multigene Prädisposition für Adipositas aufweist. Bei einer ausgeglichenen Energiebilanz spielt diese jedoch kaum eine Rolle. Kommen allerdings Bewegungsmangel oder Überernährung hinzu, wirkt es sich bei prädisponierten Menschen stärker aus als bei jenen, die diese Veranlagung nicht besitzen. [Hofbauer 2002]:

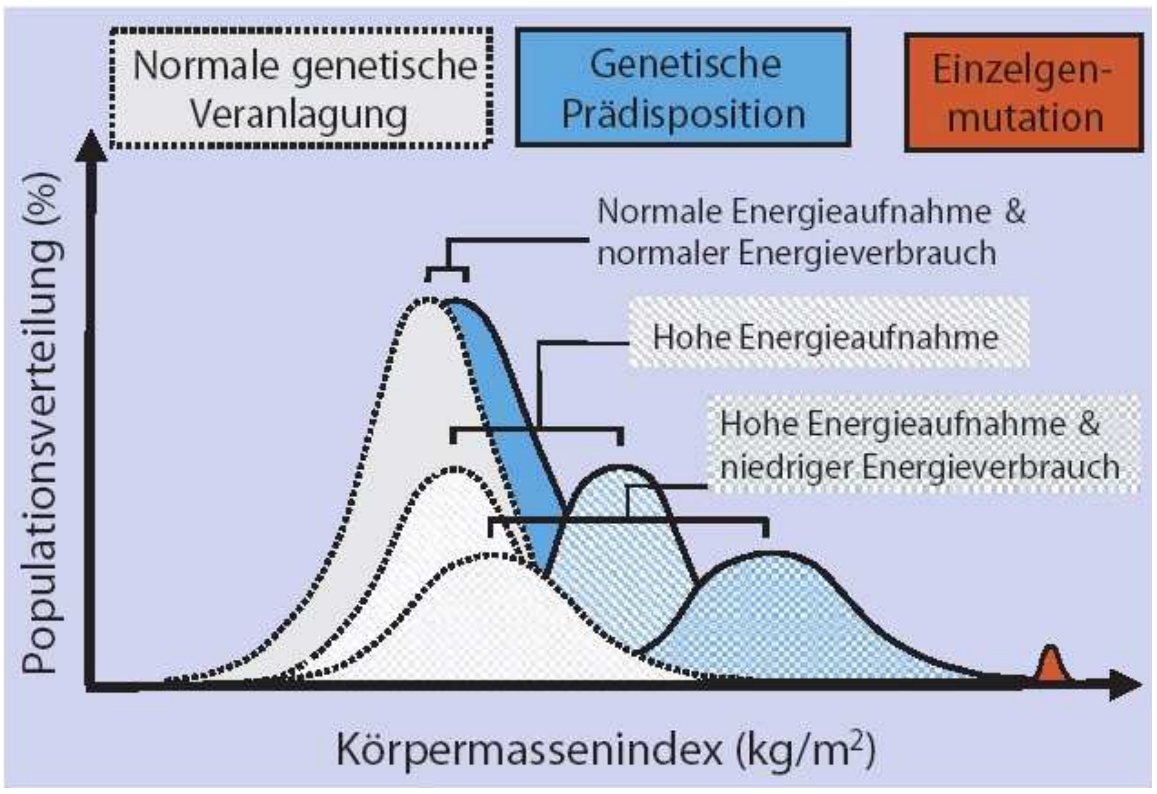

Abbildung 4: Multigene Vererbung von Adipositas [Hofbauer 2002, S.938] 
In Zwillingsstudien konnte eine hohe Korrelation des BMI sowohl bei zusammen als auch bei getrennt lebenden eineiigen Zwillingen gefunden werden. Der Korrelationskoeffizient variierte zwischen 0,66 und 0,74. Umweltfaktoren, wie das Alter und der Grad der Trennung, hatten hierbei keinen Einfluss. Bei heterozygoten Zwillingen zeigte sich hingegen lediglich ein Korrelationskoeffizient zwischen 0,15 und 0,33 [Stunkard et al. 1990].

\subsection{Monogener Vererbungsmodus}

Mutationen der Gene, die für Botenstoffe des Hunger-Sättigungs-Kreislaufes codieren, sind durch eine schwere Early Onset Adipositas mit Hyperphagie charakterisiert [Farooqi 2007].

Defekte an Leptin oder dem Leptinrezeptor zeichnen sich durch eine zusätzliche Immunschwäche, die durch eine verminderte T-Lymphozytenzahl und -funktion zustande kommt, aus. Therapeutisch kann fehlendes Leptin substituiert werden, was zu einer Normalisierung des Essverhaltens führt [Farooqi 2007].

Eine POMC-Defizienz äußert sich neonatal in einer Addison-Krise, da POMC das gemeinsame Vorläufermolekül von ACTH, MSH und aMSH ist. Die Kinder sind auf Grund des MSH-Mangels hellhäutig und rothaarig. Die heterozygoten Eltern zeigen ebenfalls einen erhöhten BMI, was auf eine Dosis-Wirkungs-Beziehung schließen lässt [Krude et al. 2003].

Eine Therapie ist nicht verfügbar [Farooqi 2007], Therapieversuche durch Substitution mit dem Melanocortinfragment ACTH 4-10 und Schilddrüsenhormonen schlugen fehl [Krude et al. 2003].

Die häufigste monogene Ursache für Adipositas in Europa ist der MC4-Rezeptor-Defekt. Er kommt bei $0,5-1 \%$ der adipösen Erwachsenen und bei $6 \%$ der Personen mit extremer Adipositas mit Beginn in der Kindheit vor. Es sind unterschiedliche Mutationen bekannt, die zu dem Defekt führen [Farooqi 2007]. Insgesamt ist die Hyperphagie nicht so schwer wie beim Leptin-Rezeptor-Defekt [Farooqi 2007].

Die Patienten haben eine erhöhte Gesamtkörpermasse und eine erhöhte Wachstumsgeschwindigkeit durch eine frühe Hyperinsulinämie [Farooqi 2007]. Auch hier gibt es bisher keinen therapeutischen Ansatz [Farooqi 2007]. 


\subsection{Adipositas-Syndrome}

Leitsymptome aller adipogenen Syndrome sind geistige Entwicklungsverzögerung, Hypogonadismus und dysmorphe Stigmata [Reinehr und Wabisch 2006].

Das häufigste adipogene Syndrom ist das Prader-Willi-Syndrom. Laut Studien wird die Häufigkeit mit 1:25000 Geburten angegeben. Experten vermuten, dass das Krankheitsbild unterdiagnostiziert wird und schätzen die wahre Inzidenz auf 1:10 000- 1:15 000 [Chen et al. 2007].

Häufigste Ursache ist eine Mikrodeletion des kurzen Armes des väterlichen Chromosoms 15. Das Chromosom unterliegt dem Imprinting, was in diesem Fall bedeutet, dass nur das väterliche Allel exprimiert wird. Selten kann auch ein Fehler im Imprintingmechanismus selbst verantwortlich sein [Chen et al. 2007].

Charakteristischerweise zeigen sich neben dysmorphen Stigmata wie Mandelaugen [Chen et al. 2007] und schmaler Oberlippe [Chen et al. 2007] eine Hypopigmentierung von Haut, Haaren und Augen [Chen et al. 2007], Stammfettsucht [Witkowski et al. 1999], Hypogonadismus und -genitalismus [Witkowski et al. 1999], Hodenhochstand [Reinehr und Wabisch 2006] und Strabismus [Witkowski et al. 1999].

Vor allem die ausgeprägte Muskelhypotonie als Säugling („Floppy Infant“), die mit einer Trinkschwäche und verzögerter motorischer Entwicklung [Reinehr und Wabisch 2006] einhergeht, und die Hyperphagie, die zumeist im ersten bis zweiten Lebensjahr einsetzt, sind pathognomonisch [Chen et al. 2007].

Betroffene Kinder zeigen außerdem Entwicklungsverzögerungen im Bereich der Motorik und Sprache. Sie kommen durchschnittlich mit 12 Monaten zum Sitzen und erlernen das freie Laufen mit 24 Monaten. Ihr erstes Wort sprechen sie im Alter von 18 Monaten bis sechs Jahren. Der mittlere IQ beträgt 60-70. Die Patienten können aber auch einen normalen IQ aufweisen [Chen et al. 2007].

Vegetative Symptome sind Hyposalivation, erhöhte Schmerztoleranz, Tagschläfrigkeit, Temperaturregulationsprobleme und ein verminderter Grundumsatz [Chen et al. 2007].

Dieser verminderte Grundumsatz, die Unfähigkeit, sich zu erbrechen, Lethargie und ein Mangel an Wachstumshormon (GH) führen zu einem Kalorienbedarf von lediglich 1000-1200 kcal pro Tag [Chen et al. 2007].

Eine kausale Therapie ist nicht verfügbar, aber es hat sich gezeigt, dass die Kinder von einer GH-Substitution im Bezug auf Endlänge und Körperfettanteil (-10\%) profitieren [Chen et al. 2007].

Die Ernährung muss ein Leben lang streng überwacht werden. Kleinkinder erhalten 800-900 $\mathrm{kcal} / \mathrm{Tag}$, was mit dem Alter bis auf maximal $1200 \mathrm{kcal}$ steigerbar ist [Chen et al. 2007]. 
Weitere seltene adipogene Syndrome sind in Tabelle 2 zusammengefasst.

\begin{tabular}{|l|l|}
\hline Seltene adipogene Syndrome & \multicolumn{1}{l|}{$\begin{array}{l}\text { Symptome } \\
\text { mentale Retardierung, Hypogonadismus und }\end{array}$} \\
\hline Laurence-Moon-Biedel-Bardet-Syndrom & Zahnunterzahl \\
& Nierenhypoplasie \\
& Kleinwuchs \\
& Postaxiale Hexadaktylie \\
& Retinitis pigmentosa \\
\hline Cohen-Syndrom & Kleinwuchs \\
& Schmale Hände/ Füße mit langen Zehen/ Fingern \\
& Offener Mund mit vorstehenden Schneidezähnen \\
& Mikrozephalus \\
\hline Sotos-Syndrom & hohes Geburtsgewicht \\
& Hypertelorismus \\
& Längliches Gesicht \\
& Akzeleriertes Knochenalter \\
& Große Hände/ Füße \\
\hline Weaver-Syndrom & Makrozephalie \\
& Rundes Gesicht \\
& Fingerspitzenpolster \\
\hline Alström-Syndrom & Hörschwäche \\
& Sehverlust \\
& Diabetes mellitus Typ 2 \\
\hline
\end{tabular}

Tabelle 2: Symptome seltener Adipogener Syndrome [Reinehr und Wabisch 2006, S.69]

\subsubsection{Psychiatrische Grunderkrankungen}

Eine Reihe psychiatrischer Grunderkrankungen und Verhaltensstörungen können mit Adipositas einhergehen [Warschburger 2006].

\begin{tabular}{|l|l|}
\hline Erkrankung & Leitsymptome \\
\hline Essstörungen & $\begin{array}{l}\text { Kontrollverlust beim Essen } \\
\text { Selbstinduziertes Erbrechen }\end{array}$ \\
\hline Anpassungsstörung, Depression & $\begin{array}{l}\text { Schlafstörungen } \\
\text { Traurigkeit } \\
\text { Sozialer Rückzug } \\
\text { Generalisiert negatives Selbstbild }\end{array}$ \\
\hline Angststörung & $\begin{array}{l}\text { Vermeidungsverhalten } \\
\text { Bauch- oder Kopfschmerzen }\end{array}$ \\
\hline $\begin{array}{l}\text { Posttraumatische Belastungsstörung } \\
\text { (PTBS) }\end{array}$ & $\begin{array}{l}\text { Albträume } \\
\text { Schreckhaftigkeit } \\
\text { Interessenverlust } \\
\text { Leistungsabfall }\end{array}$ \\
\hline Dissoziales Verhalten & $\begin{array}{l}\text { Stehlen } \\
\text { Aggressives Verhalten } \\
\text { Missachtung von Regeln } \\
\text { (Schuleschwänzen, } \\
\text { Drogenmissbrauch) }\end{array}$ \\
\hline
\end{tabular}

Tabelle 3: Psychiatrische Ursachen für Adipositas [nach Warschburger 2006] 


\subsubsection{Endokrinologische Grunderkrankungen}

Leitsymptome einer sekundären Adipositas bei endokrinologischer Ursache sind Kleinwuchs, verminderte Wachstumsgeschwindigkeit und eine schnelle Gewichtszunahme [Reinehr und Wabisch 2006].

\subsection{Hypothyreose}

Die wichtigsten endokrinologischen Ursachen für ein erhöhtes Körpergewicht sind die Hypothyreose, Wachstumshormon-Mangel, Morbus Cushing und das Cushing Syndrom [Reinehr und Wabisch 2006], wobei die häufigste die Hypothyreose, bei Kindern meist immunologisch bedingt, ist (Hashimoto-Thyreoiditis). Sie kommt bei 1-2:1000 Schulkindern vor und führt zu einem herabgesetzten Stoffwechsel und Grundumsatz [Reinehr und Wabisch 2006]. Symptome sind Obstipation, Müdigkeit, mangelnde Konzentrationsfähigkeit, Hypercholesterinämie und in extremen Fällen das Myxödem [Reinehr und Wabisch 2006].

\subsection{Wachstumshormonmangel}

Ein Mangel an Wachstumshormon (GH) oder seinem Rezeptor kommt bei 1:400010000 Kindern vor. Der GH-Magel zeichnet sich durch eine vermehrte Fettmasse, aufgrund der fehlenden lipolytischen und antilipogenetischen Eigenschaften von $\mathrm{GH}$, aus [Reinehr und Wabisch 2006].

Das Leitsymptom stellt vor allem die verminderte Wachstumsgeschwindigkeit dar. Kleine Hände und Füße und schwach ausgebildete Muskulatur sind ein häufiger Nebenbefund [Reinehr und Wabisch 2006].

\subsection{Hyperkortisolismus}

Morbus Cushing und das Cushing-Syndrom sind selten und Folge eines hormonproduzierenden Tumors der Hypophyse oder Nebennierenrinde [Reinehr und Wabisch 2006].

Glukokortikoide stimulieren sowohl die Bildung von Fett in vorhandenen Adipozyten als auch ihre Neubildung [Reinehr und Wabisch 2006].

Die Erkrankung zeichnet sich durch vielfältige Symptome aus. In diesem Zusammenhang stellen die schnelle Gewichtszunahme, das typische Vollmondgesicht, die Stammfettsucht, der Stiernacken und ein Wachstumsstillstand die entscheidenden klinische Zeichen dar [Reinehr und Wabisch 2006, Herold 2007, Sitzmann 2007]. 


\subsubsection{Andere Erkrankungen}

Immobilisierende Erkrankungen oder solche, die mit vermindertem Wachstum einhergehen, können generell zu einem erhöhten BMl führen, wie zum Beispiel Querschnittslähmung als Folge einer Spina bifida oder eines Traumas und die Achondroplasie [Reinehr und Wabisch 2006]. In einem Review von 38 Studien zeigt sich, dass die Prävalenz von Adipositas bei behinderten Kindern etwa doppelt so hoch ist wie bei den gesunden Altersgenossen [Reinehr et al. 2010].

\section{TABLE 1}

Prevalences of overweight and obesity in disabled children and adolescents

\begin{tabular}{|c|c|c|c|}
\hline \multicolumn{4}{|c|}{ a) Compared with healthy children and adolescents } \\
\hline Disability & $\begin{array}{l}\text { Percentage of overweight/ } \\
\text { obesity }\end{array}$ & Healthy comparison group & Country \\
\hline Functionally restricted mobility & $30 \%$ overweight & $16 \%$ overweight & USA (11) \\
\hline Developmental delay & $\begin{array}{l}24 \% \text { overweight } \\
15 \% \text { obese }\end{array}$ & $\begin{array}{l}17 \% \text { overweight } \\
6 \% \text { obese }\end{array}$ & Australia (12) \\
\hline Learning disability & $\begin{array}{l}35 \% \text { overweight } \\
21.9 \% \text { obese }\end{array}$ & $\begin{array}{l}31 \% \text { overweight } \\
15.7 \% \text { obese }\end{array}$ & USA (11) \\
\hline Learning disability & $19.3 \%$ obese & $12.2 \%$ obese & USA(13) \\
\hline Hearing or visual impairment & $18.4 \%$ obese & & \\
\hline Autism & $23.4 \%$ obese & & \\
\hline $\begin{array}{l}\text { Attention deficit (hyperactivity) } \\
\text { disorder }\end{array}$ & $18.9 \%$ obese & & \\
\hline Asthma & $19.7 \%$ obese & & \\
\hline Asthma & $24.6 \%$ overweight & $14.2 \%$ overweight & Denmark (14) \\
\hline \multicolumn{4}{|c|}{ b) Proportions in individual disabilities without comparison group *1 } \\
\hline Cerebral palsy (15) & \multicolumn{3}{|l|}{$29 \%$ overweight $^{2}$} \\
\hline Visual impairment (16) & \multicolumn{3}{|l|}{$25.8 \%$ overweight, $11.8 \%$ obese } \\
\hline $\begin{array}{l}\text { Attention deficit (hyperactivity) } \\
\text { disorder (17) }\end{array}$ & \multicolumn{3}{|l|}{$29 \%$ overweight, $17 \%$ obese } \\
\hline Autism (17) & \multicolumn{3}{|l|}{$35.7 \%$ overweight, $19 \%$ obese } \\
\hline Spina bifida (18) & \multicolumn{3}{|l|}{$18 \%$ obese } \\
\hline \multicolumn{4}{|c|}{$\begin{array}{l}\text { c) Other disabilities that are associated with overweight/obesity, but no prevalence data are available } \\
\text { from the literature }\end{array}$} \\
\hline \multicolumn{4}{|l|}{ - Prader-Willi syndrome (e24) } \\
\hline \multicolumn{4}{|l|}{ - Down's syndrome (e46) } \\
\hline \multicolumn{4}{|l|}{ - Muscular dystrophy (e47) } \\
\hline \multicolumn{4}{|l|}{ - Type I diabetes (e22) } \\
\hline \multicolumn{4}{|l|}{ - Juvenile arthritis (e22) } \\
\hline \multicolumn{4}{|c|}{ - Mental retardation (e21, e30, e48, e49) } \\
\hline
\end{tabular}

${ }^{\star 1}$ To provide a comparison: In Germany, $8.7 \%$ of children are overweight, and an additional $6.3 \%$ are obese (e11) $\star^{2}$ Other studies have reported a $50 \%$ reduced risk of overweight (e3) 
Einen besonderen Stellenwert hat noch der so genannte hypothalamische Symptomkomplex, meist Folge einer Operation oder Bestrahlung der hypothalamischen Region (z.B. bei Kraniopharyngeom). Die Ursache für die Hyperphagie der Patienten ist noch nicht gänzlich geklärt. Diskutiert wird zurzeit ein Verlust des Sättigungsgefühls durch Leptinresistenz [Reinehr und Wabisch 2006].

\subsubsection{Medikamenteninduzierte Adipositas}

Viele Medikamente führen zu einer Gewichtszunahme, wichtige Vertreter sind:

Antidepressiva, Neuroleptika, Insulin, Glitazone, Cortisol und Östrogene [Reinehr und Wabisch 2006].

\subsubsection{Primäre Ursachen Lifestyle}

Die Energiebilanz bezeichnet das Verhältnis von Energieaufnahme und- verbrauch, welcher sich wiederum aus Grundumsatz und körperlicher Aktivität zusammensetzt. Das bedeutet, um eine ausgeglichene Energiebilanz zu erreichen, gibt es zwei modellierbare Größen: Ernährung und Bewegung [Kersting 2006].

\subsubsection{Ernährung}

Schon eine positive Energiebilanz von nur $50 \mathrm{kcal}$ am Tag führt über ein halbes Jahr zur Gewichtszunahme von einem Kilogramm und wird von dem großen und vielfältigen Angebot an energiedichten Lebensmitteln begünstigt. $75 \%$ des täglichen Fettverzehrs stammt in Deutschland aus so genannten versteckten Fetten in Wurst oder Gebäck. Getränke haben oft einen großen Anteil an einem zu hohen Zuckerverzehr [Kersting 2006].

Das Forschungsinstitut für Kinderernährung in Dortmund (FKE), die Deutsche Gesellschaft für Ernährung (DGE) und der Infodienst Verbraucherschutz-Ernährung-Landwirtschaft haben ein System entwickelt, nach dem Lebensmittel in Gruppen eingeteilt werden, je nachdem wie häufig man sie verzehren sollte.

Reichlich empfohlen werden pflanzliche Lebensmittel wie Obst und Gemüse, Getreideprodukte, Nudeln, Reis und Kartoffeln, aber auch Wasser und kalorienarme Getränke.

Tierische Produkte wie Milch und Milchprodukte, Fleisch und Fleischprodukte sowie Eier sollten nur mäßig konsumiert werden.

Zurückhaltung ist beim Konsum von Speisefetten, fetten Snacks, Knabberartikeln und Süßwaren geboten [Mensink et al. 2005]. 


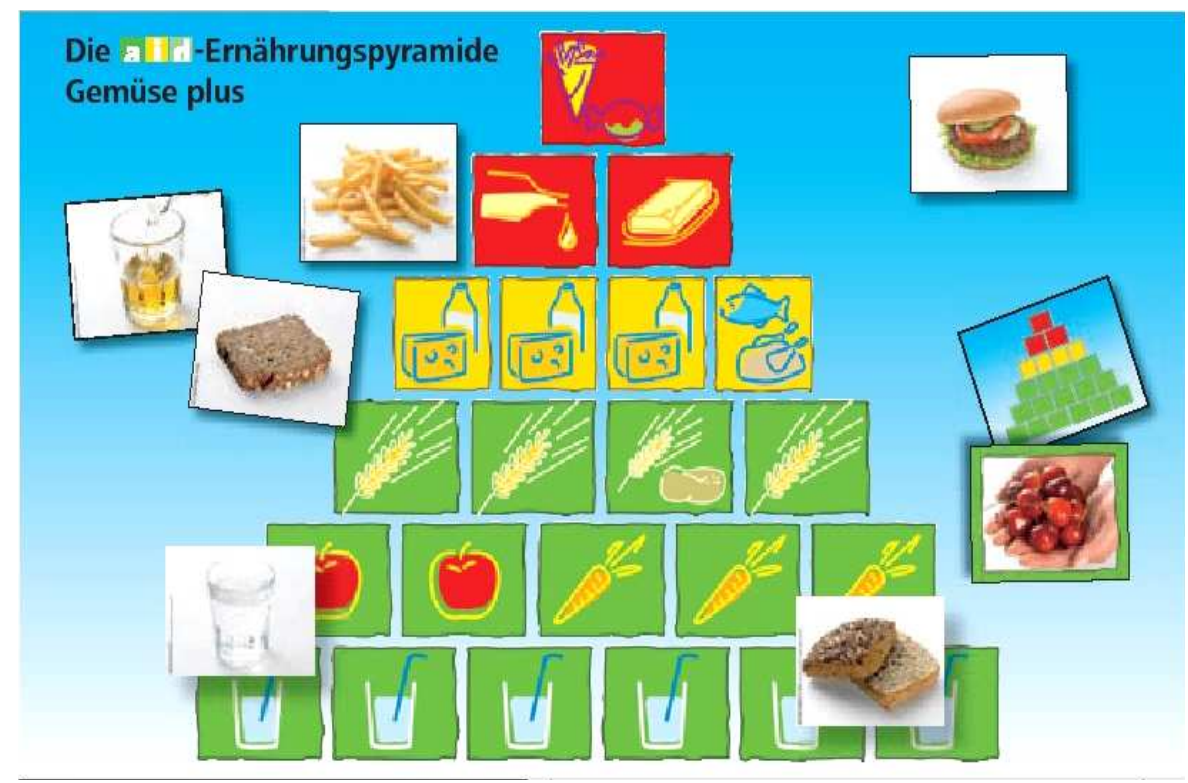

Abbildung 6: Die aidErnährungs-Pyramide: mit den Farben der Ampel soll verdeutlicht werden, was in welchen Mengen Verzehrt werden soll. [Infodienst Verbraucherschutz • Ernährung • Landwirtschaft e. $V$. (aid) 2008]

Für die KiGGS-Studie wurden die Ernährungsgewohnheiten der Teilnehmer anhand eines Food Frequency Questionnaire erfragt und nach verschiedenen Gesichtspunkten, wie Alter und Geschlecht, sozialem Status und Migrationshintergrund, ausgewertet [Mensink et al. 2007].

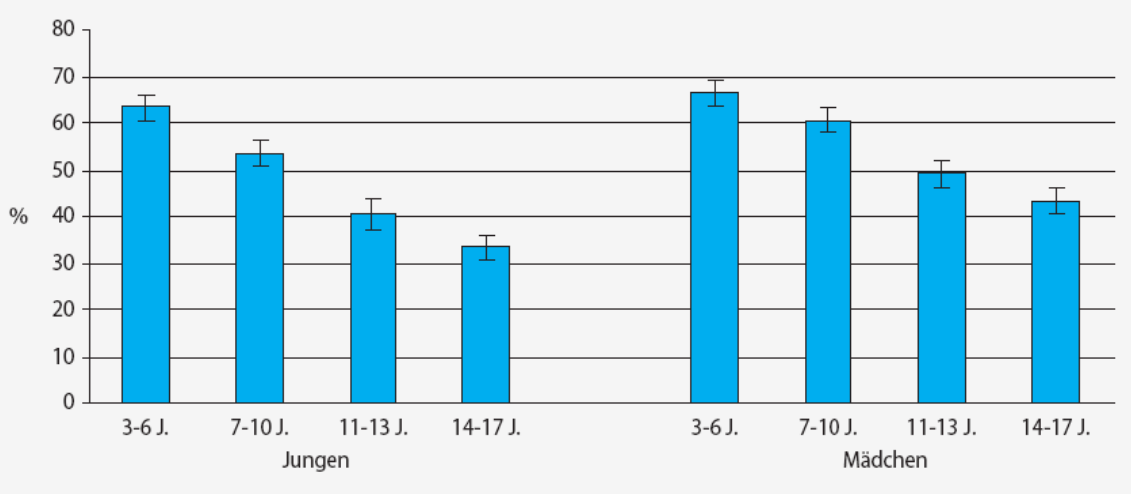

Abbildung 7: Anteil der Kinder, die täglich frisches Obst essen: Prävalenz und 95\%Konfidenzintervall. [Mensink et al. 2007, S.618]

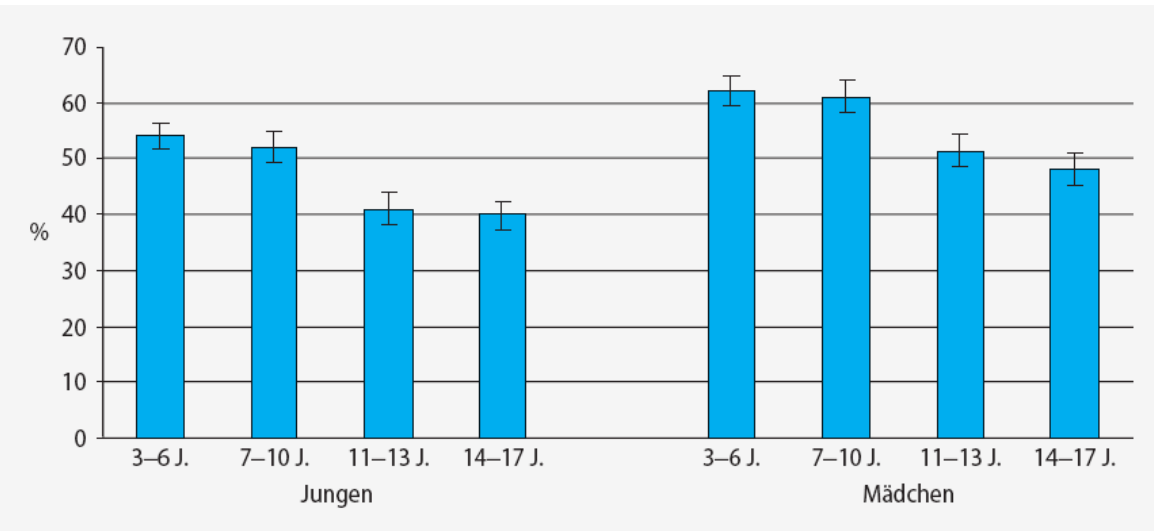

Abbildung 8: Anteil der Kinder, die täglich gekochtes Gemüse Tiefkühlgemüse, Konservengemüse und Blattsalat, Rohkost oder rohes Gemüse konsumieren. [Mensink et al. 2007, S. 619] 
Am Beispiel von Obst und Gemüse lässt sich gut zeigen, was die Studie insgesamt herausbrachte: Mädchen ernähren sich tendenziell etwas gesünder und ausgewogener als Jungen, was auch beim Konsum von Fast Food (z.B. Bratwurst, Currywurst, Hamburger, Döner Kebab), Fertiggerichten und Softdrinks auffällt [Mensink et al. 2007].

\begin{tabular}{|l||r|r||r|r|}
\hline \multicolumn{4}{|c|}{$1-6 x /$ Woche } & \multicolumn{2}{c|}{ mehrmals täglich } \\
\multicolumn{1}{|||}{} & Mädchen & \multicolumn{1}{l}{ Jungen } & Mädchen & Jungen \\
\hline Fast Food & $9 \%$ & $18 \%$ & & \\
\hline Fertiggerichte & $33 \%$ & $25 \%$ & & \\
\hline Softdrinks & & & $19,80 \%$ & $25,20 \%$ \\
\hline Obst & & & $24,10 \%$ & $17,80 \%$ \\
\hline Gemüse roh & & & $5,90 \%$ & $3,70 \%$ \\
\hline Mineralwasser & & & $42,90 \%$ & $38,90 \%$ \\
\hline
\end{tabular}

Tabelle 4: Verzehr unterschiedlicher Lebensmittelgruppen [nach Mensink et al. 2007, S.612f]

Jüngere Kinder essen etwas gesünder und ausgewogener als Jugendliche. Sie konsumieren weniger Erfrischungsgetränke, Fleisch, Wurst und Fast Food, dafür mehr Milch, Fisch, Obst und Gemüse, aber auch etwas mehr Süßigkeiten als Ältere [Mensink et al. 2005].

Die Gruppe, die sich am ungesündesten ernährt, sind die Jungen von 14-17 Jahren [Mensink et al. 2005].

Das Wissen über richtige Ernährung korreliert nicht mit dem BMI, sondern mit dem eigenen Bildungsstand des Kindes und dem der Mutter [Kersting 2006].

Kinder und Jugendliche sind eine beliebte Zielgruppe für Hersteller energiedichter Lebensmittel. In Australien betrifft einer von drei Werbespots im Kinderfernsehprogramm Nahrungsmittel, die mit einem erhöhten Risiko für Übergewicht und Adipositas einhergehen (Junk Food) und im Widerspruch zu den Empfehlungen von Fachgruppen stehen. Ähnliche Trends wurden auch in Neuseeland, den USA und Großbritannien festgestellt [Dixon et al. 2007].

Durch die Werbung kommt zu einer ohnehin adipogenen Tätigkeit wie dem Fernsehen [Graf und Dordel 2006] auch noch ein gesteigertes Verlangen nach Lebensmitteln, die zusätzlich zum verminderten Energieverbrauch zu einer erhöhten Energieaufnahme führen, hinzu [Dixon et al. 2007].

Dixon et al. präsentierten in einem Experiment Kindern im Alter von etwa 10 Jahren ein Kinderprogramm, in dem zu gleichen Teilen für Junk Food und gesundes Essen geworben wurde. Die Popularität des Junk Foods blieb zwar gleich hoch, aber die des gesunden Essens konnte deutlich verbessert werden, so dass eine Dosis-Wirkungs-Beziehung vermutet wird. 


\subsubsection{Körperliche Aktivität}

Eine angemessene und vielfältige körperliche Aktivität bei Kindern ist der Katalysator für die motorische Entwicklung und Entwicklung des Bewegungsapparates [Dordel 2007]. Sie beugt der Entstehung von Übergewicht [Lampert et al. 2007a, Hampson et al. 2007] oder anderen chronischen Erkrankungen [Lampert et al. 2007a] vor. Endogene (Muskelkraft) und exogene (Schwerkraft) Kräfte stellen außerdem einen Wachstumsreiz dar [Dordel 2007].

Der motorische Entwicklungsstand eines Schulkindes bei der Einschulung ist stark von der Bewegungserfahrung abhängig, die es bis dahin sammeln konnte, und somit höchst variabel [Dordel 2007]. Kinder, die sich wenig bewegen, zeigen messbare motorische Defizite [Graf und Dordel 2006]. Im Alter von 7-12 Jahren ist die Koordinationsfähigkeit besonders gut trainierbar (sensible Phase), was Kindern eine Chance gibt, eventuell vorhandene Defizite auszugleichen [Dordel 2007].

Während und nach der Pubertät nimmt das Interesse an körperlicher Aktivität, besonders bei Mädchen, ab [Dordel 2007, Lampert et al. 2007a, Hampson et al. 2007]. Insgesamt treiben Mädchen weniger Sport als Jungen [Lampert et al. 2007a, Metcalf et al. 2008]. Die Folge kann eine Verschlechterung von Körperhaltung und Verlust bereits erworbener koordinativer Fähigkeiten sein [Dordel 2007].

Die KiGGS-Studie ergab, dass ein Drittel der Mädchen und ein Sechstel der Jungen im Alter von 14-17 Jahren körperlich inaktiv sind, das heißt sich weniger als ein Mal in der Woche körperlich im oder außerhalb des Sportvereins betätigen [Lampert et al. 2007a].

In einer Analyse des Bewegungsalltags von 1000 Kindern im Alter von 6-10 Jahren zeigte sich, dass sie im Durchschnitt nur eine Stunde am Tag körperlich aktiv sind und sich 15-30 Minuten intensiv bewegen. Den größten Teil des Tages verbringen sie im Liegen, Sitzen (je 9 Stunden) oder Stehen (5 Stunden) [Bös 2001]. Für Erwachsene lautet die Empfehlung, sich mindestens drei Mal in der Woche moderat körperlich zu betätigen. Die Richtlinien der Weltgesundheitsorganisation empfehlen jedoch für Kinder moderate bis intensive körperliche Aktivität für 60 Minuten pro Tag [Metcalf et al. 2008].

Laut KiGGS-Studie wird diese Empfehlung bei den 3-10-Jährigen von immerhin 36,1\% erfüllt, allerdings treiben auch ein Viertel aller Kinder dieser Altersgruppe nicht regelmäßig, ein Achtel niemals Sport [Lampert et al. 2007a].

Ein weiteres Problem stellt der allgemeine Trend zur passiven Fortbewegung mit Auto, Bus und Bahn dar. In Amerika sank zum Beispiel der Anteil der Kinder, die zu Fuß zur Schule gehen oder mit dem Rad dort hinfahren, zwischen 1969 und 2003 von 50\% auf 15\% ab [Davis et al. 2007]. Auch in Deutschland nimmt die Bewegung im Alltag bei Kindern ab, was sich in der körperlichen Leistungsfähigkeit abbildet: zwischen 1980 und 2000 gingen 
Ausdauer, Sprungkraft und Flexibilität bei deutschen Kindern um 10-20\% zurück [Graf und Dordel 2006].

Der Aktivitätslevel eines Kindes hängt von vielen verschiedenen Faktoren ab: von der Aktivität der Eltern, der Unterstützung durch Familie und Peer Group, Spielmöglichkeiten in Nachbarschaft und Schule [Davis et al. 2007], dem sozialen Image des Sportlers beim Kind [Hampson et al. 2007] und dem eigenen sportlichen Erfolg [Graf und Dordel 2006].

Deshalb sollte das Ziel von Interventionen sein, das Image des Sportlers bei Kindern zu stärken und zeitliche und räumliche Freiräume zu schaffen, die nur für die Bewegung reserviert sind [Hampson et al. 2007].

Inaktive Tätigkeiten, wie Fernsehen, PC oder Konsole spielen, sind mit körperlicher Inaktivität und Übergewicht und Adipositas assoziiert [Lampert et al. 2007b].

Bei einem Fernsehkonsum von 3-4 Stunden am Tag steigt das Adipositasrisiko bei Kindern um das Vier- bis Fünffache [Graf und Dordel 2006]. Das betrifft laut KiGGS-Studie 22,1\% der Jungen und 23,6\% der Mädchen. Zusätzlich verbringen 16,9\% der Jungen und 6,4\% der Mädchen mehr als 3 Stunden am Tag vor dem PC und 6,1\% der Jungen und 1,1\% der Mädchen mehr als 3 Stunden täglich an der Spielkonsole [Lampert et al. 2007b]. Bei Mädchen ist eher der Konsum von PC und Internet mit einem erhöhten Risiko für Adipositas assoziiert, bei Jungen mehr das Fernsehen [Lampert et al. 2007b].

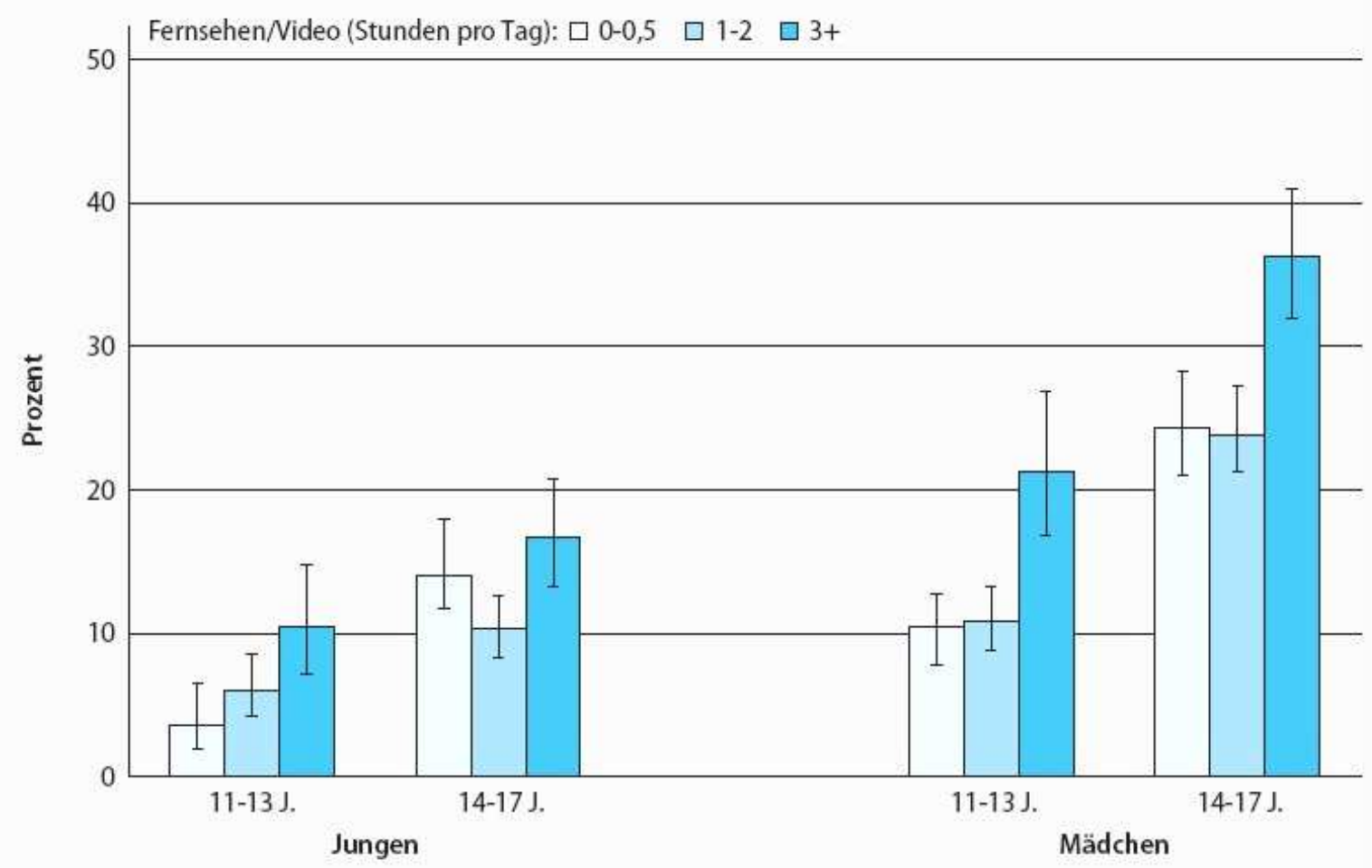

Abbildung 9: Zusammenhang zwischen körperlicher Inaktivität (<1Mal/Woche Körperliche Aktivität) und PC-/Fernsehkonsum in der KiGGS-Studie [Lampert et al. 2007b, S. 650] 


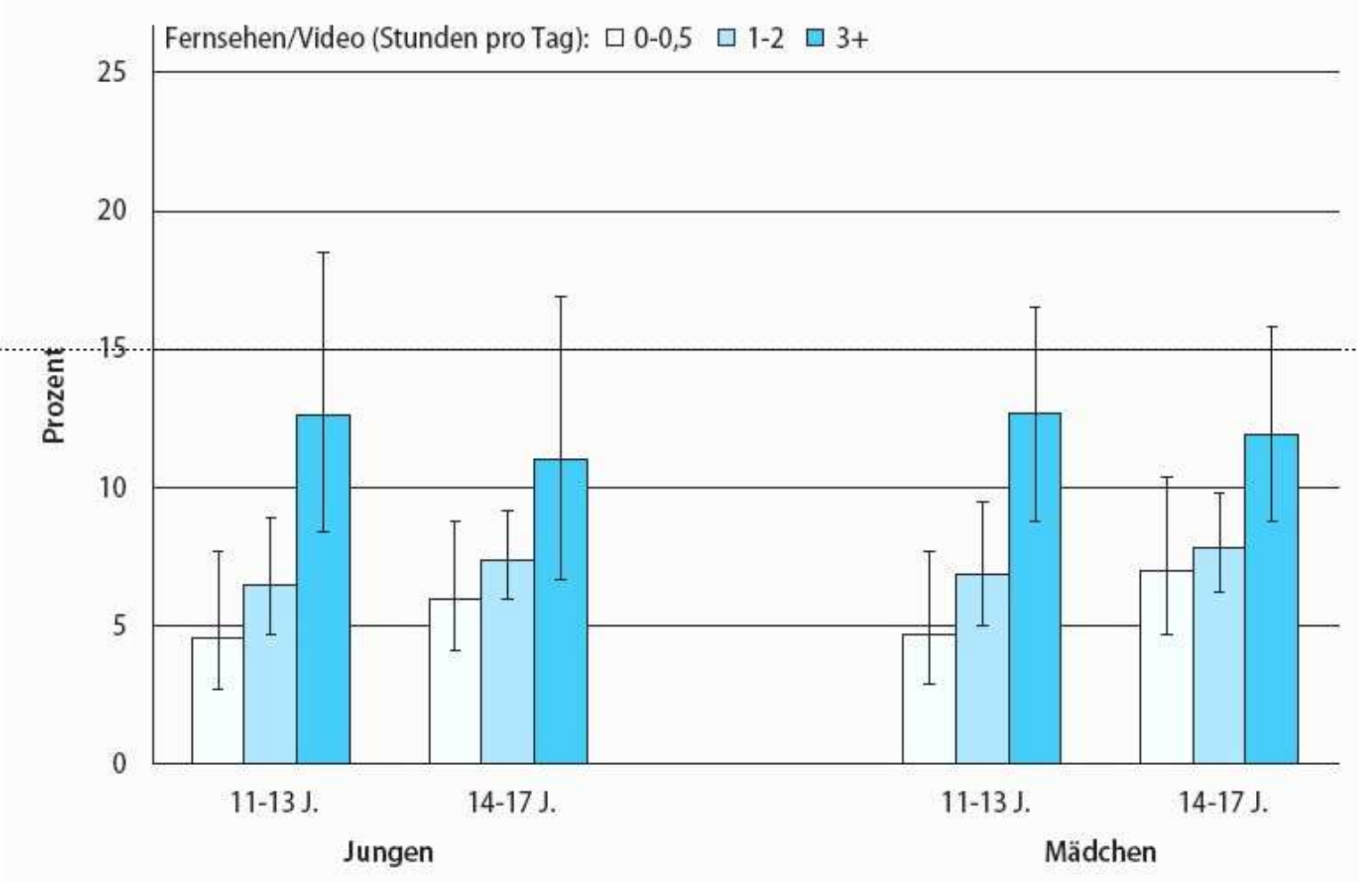

Abbildung 10: Zusammenhang zwischen Adipositas und PC-/ Fernsehkonsum in der KiGGS-Studie [Lampert et al. 2007b, S.650]

\subsubsection{Weitere Einflussfaktoren}

\subsection{Familie}

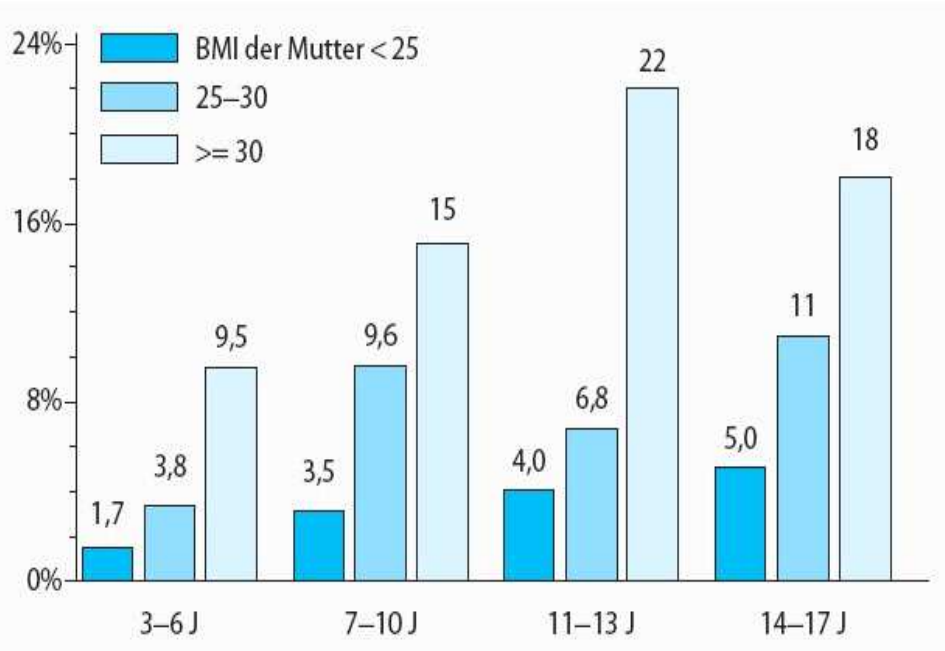

Die Lebensumstände der Familie spielen eine extrem wichtige Rolle für die Entwicklung des Ess- und Bewegungsverhaltens eines Kindes. Der mütterliche $\mathrm{BMI}$ ist ein starker Prädiktor für den BMI ihrer Kinder [Röbl et al. 2008, Kurth und SchaffrathRosario 2007, Watkins et al. 2007, Kleiser et al. 2009, Kries et al. 2002].

Abbildung 11: Prävalenz von Adipositas nach Alter der Kinder und BMI der Mutter in der KiGGSStudie [Kurth und Schaffrath-Rosario 2007, S.740] 
Nur eine von fünf adipösen Müttern mit adipösen Kindern nimmt sich und ihr Kind auch als solches wahr. Selbst nach der Aufnahme der Kinder in eine Spezialklinik beschrieben Mütter ihre Kinder lediglich als übergewichtig und nicht als adipös und waren sich über den Krankheitswert nicht bewusst. Dieser Effekt war umso größer, je jünger die betroffenen Kinder waren [Watkins et al. 2007].

Weitere familiäre Risikofaktoren für die Entstehung von Adipositas sind Einzelkindstatus [Röbl et al. 2006], überfürsorgliche Eltern [Lissau und Sørensen 1994], Kinder, die Nachzöglinge sind [Lissau und Sørensen 1994], Leben mit allein erziehendem Elternteil [Kries et al. 2002], Kinder, deren Eltern rauchen [Kleiser et al. 2009, Kries et al. 2002], mütterliches Rauchen während der Schwangerschaft [Kries et al. 2002], Kinder als Ergebnis ungewollter Schwangerschaften [Lissau und Sørensen 1994] und geringe Bindung an die Eltern bis hin zur Deprivation [Röbl et al. 2006].

Allein das Fehlen geregelter Familienmahlzeiten kann zur Entstehung von Adipositas beitragen [Kries et al. 2002]. Gemeinsame Mahlzeiten sind mit einer ausgewogeneren Ernährungsweise assoziiert [Davis et al. 2007].

90\% der KiGGS-Teilnehmer nehmen das Abendessen im Kreis der Familie ein, aber nur 50\% auch das Frühstück und Mittagessen [Kersting 2006].

Ein Viertel verlässt das Haus sogar ohne Frühstück und investiert mitgebrachtes Essengeld häufig in energiedichte Snacks [Kersting 2006], dabei ist das Auslassen des Frühstücks, ganz besonders bei Jungendlichen, ein eigenständiger Risikofaktor für Übergewicht und Adipositas [Davis et al. 2007].

Ein weiterer unabhängiger Risikofaktor, der eng mit dem Erziehungsstil der Eltern zusammenhängt, ist der Schlafmangel [Nixon et al. 2008, Heins et al. 2007, Kleiser et al. 2009].

Für Grundschulkinder wird eine Schlafdauer von etwa 10 Stunden empfohlen [Heins et al. 2007].

Unter chronischem Schlafmangel kommt es unter anderem zu einem Absinken des Leptinund einer Erhöhung des Ghrelinspiegels, was zu gesteigertem Appetit führt [levers-Landis und Redline 2007].

\subsection{Perinatale Einflüsse}

Sowohl ein hohes als auch niedriges Geburtsgewicht zählen zu den Risikofaktoren für Übergewicht und Adipositas [Röbl et al. 2006]. 
Niedriges Geburtsgewicht kann unter anderem Folge von Frühgeburtlichkeit oder Tabakkonsum der Mutter während der Schwangerschaft [Kleiser et al. 2009, Kries und Toschke 2004], besonders im ersten Trimenon [Kries et al. 2002], sein. Beides gilt als unabhängiger Risikofaktor für Adipositas [Röbl et al. 2006, Kries und Toschke 2004]. Manche dieser Kinder zeigen ein Aufholwachstum, dessen Assoziation mit späterer Adipositas in mehreren Studien gezeigt werden konnte [Ong 2007]. Bei einer schnellen Gewichtszunahme in den ersten zwei Lebensjahren (>10kg), ist das Adipositasrisiko auf das 4,3-Fache erhöht [Kries et al. 2002].

Der adipogene Einfluss eines hohen Geburtsgewichtes kann noch im 50. Lebensjahr nachgewiesen werden [Kries et al. 2002]. Die Odds Ratio beträgt $>2$ für Übergewicht und 1,56 für Adipositas [Kries et al. 2002].

Eine häufige Ursache eines hohen Geburtsgewichtes ist der Gestationsdiabetes [Gillman et al. 2003]. Die mütterliche Hyperglykämie führt beim Fetus zu einer vermehrten Ausschüttung von Insulin, was als Wachstumshormon auf den Fetus wirkt und eine vermehrte Glukoseaufnahme in die Fettzellen bewirkt [Gillman et al. 2003].

Im Tierversuch konnte auch eine Auswirkung des Hyperinsulinismus auf die Neurotransmitterexpression im Hypothalamus gezeigt werden, die zu Hyperphagie und erhöhtem Körpergewicht führte [Gillman et al. 2003].

Stillen schützt vor Adipositas. Kinder, die nicht gestillt werden, haben ein um ein Drittel erhöhtes Risiko im Gegensatz zu gestillten Kindern [Kries et al. 2002].

Laut KiGGS-Studie wurden 37,4\% der Kinder 6 Monate voll gestillt. Nicht sechs Monate voll gestillt wurden vor allem die Kinder von Raucherinnen, Müttern mit niedrigem Sozialstatus, Müttern aus den neuen Bundesländern und jungen Müttern unter dreißig Jahren [Lange $C$ et al. 2007].

Als einer der Vorteile des Stillens wird vermutet, dass das Kind die Nahrungsaufnahme selbst reguliert, ohne dass die Mutter eine Kontrollmöglichkeit hat. Außerdem kommt es, im Vergleich zur Formulanahrung, zu einer verminderten Insulinausschüttung [Kries et al. 2002]. In neuren Studien zeigte sich auch ein direkter Einfluss des Proteinanteils in der Formulanahrung auf das Körpergewicht und dem BMI bei Kleinkindern. Im Gegensatz zur Muttermilch hat Formulanahrung einen 55-80\% höheren Proteinanteil [Koletzko et al. 2009].

\subsection{Adipositas-Rebound}

Als Adipositas-Rebound (AR) bezeichnet man den Zeitpunkt in einer BMI-Kurve, an dem die negative Steigung, die nach dem ersten Lebensjahr eintritt, ihren Wendepunkt erreicht [FKE]. 
Im Vergleich zu mathematischen Verfahren erwies sich die visuelle Bestimmung des Nadirs auf der BMI-Kurve als genauestes Mittel zur Bestimmung des AR beim einzelnen Patienten [Kroke et al. 2006]. Der AR tritt zwischen dem 3. und 8. Lebensjahr [FKE], durchschnittlich im Alter von sechs Jahren bei Mädchen und 6,6 Jahren bei Jungen, auf [Williams 2005]. Kinder mit einem frühen AR tendieren zu einem höheren BMI [Williams 2005], einem akzelerierten Knochenalter, einer früheren Menarche bei Mädchen [Williams und Goulding 2008] und einem erhöhten Risiko für arterielle Hypertonie, gestörter Glukosetoleranz und Diabetes [Taylor et al. 2005]. Kinder mit frühem Rebound vor dem 5. Lebensjahr nehmen fast dreimal so schnell an Fettmasse zu, wie Kinder mit einem späten Rebound nach dem 7. Lebensjahr [Taylor et al. 2005]. Somit wird der Grundstein für Adipositas schon in der frühen Kindheit gelegt [Adair 2008].

\subsubsection{Risikogruppen}

$\mathrm{Zu}$ den Risikogruppen zählen Kinder mit niedrigem sozialen Status und Kinder mit Migrationshintergrund [Kurth und Schaffrath-Rosario 2007, Kleiser et al. 2009].

\subsubsection{Sozialstatus}

Kinder mit niedrigen sozialen Status sind öfter krank [Lange $M$ et al. 2007], sehen mehr fern, nutzen häufiger Konsolen und Computer [Lampert et al. 2007b] und sind häufiger sportlich inaktiv [Lampert et al. 2007a] als andere Kinder. Neben dem Gewichtsstatus der Eltern stellte ein niedriger sozioökonomischer Status in der KiGGS-Studie den wichtigsten Risikofaktor für Adipositas dar [Kleiser et al. 2009]. In einem Review von Shrewsbury und Wardle zeigte sich 2008 in 19 von 45 internationalen Studien (42\%) ein Zusammenhang

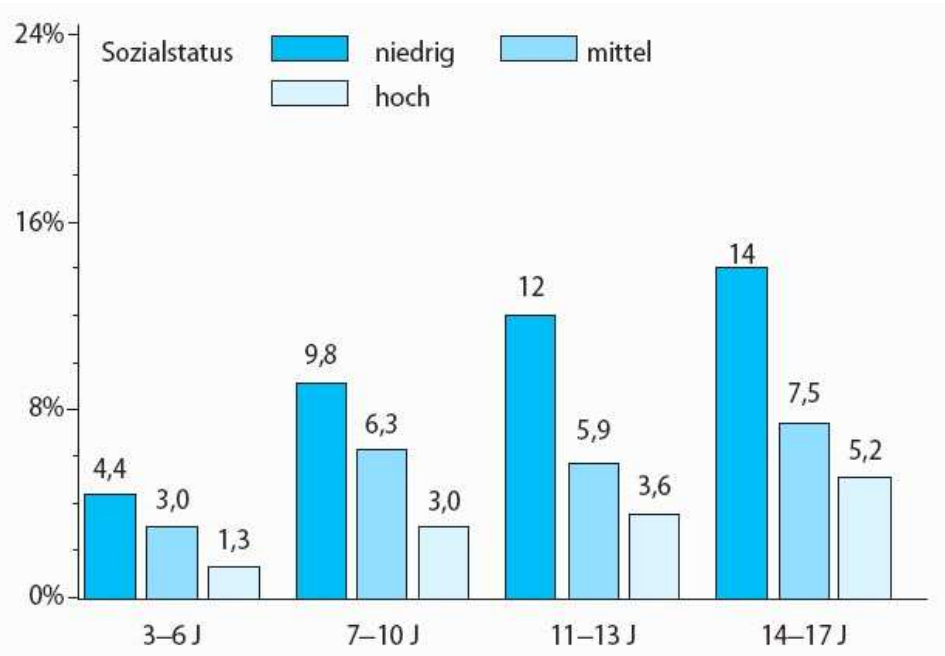
zwischen sozialen Faktoren, bei nur zwölf Studien (27\%) konnte kein Zusammenhang nachgewiesen werden. Eine neuere Studie zeigte zudem, dass Adipositas bei Kindern in niedrigen sozialen Schichten im stärkeren Maße zunimmt als in den höheren sozialen Schichten [Singh et al. 2010].

Abbildung 12: Prävalenz von Adipositas nach sozialem Status in der KiGGS- Studie [Kurth und Schaffrath-Rosario 2007, S. 740] 


\subsubsection{Migrationshintergrund}

Der Begriff „Migrant“ schließt, unabhängig von der Staatsbürgerschaft, alle diejenigen ein, deren Eltern oder sie selbst aus einem anderen Land nach Deutschland eingewandert sind [Schenk 2010].

Migrantenkinder sind im Mittel kleiner als deutsche Kinder, wiegen jedoch genauso viel [Stolzenberg et al. 2007]. Bei den Vorschulkindern sind sie 1,5 bis 2 Mal häufiger von Übergewicht und Adipositas betroffen als deutsche Kinder ohne Migrationshintergrund [Erb und Winkler 2003].

Die Jungen beschäftigen sich mehr mit elektronischen Medien als ihre deutschen Altersgenossen, bei den Mädchen ergab sich kein Unterschied zwischen beiden Gruppen [Lampert et al. 2007b].

Türkische Kinder ernähren sich ungesünder: sie trinken häufiger Softdrinks und essen mehr Süßigkeiten und Knabberartikel [Mensink et al. 2005].

Fast die Hälfte $(48,3 \%)$ der Mädchen mit Migrationshintergrund ist sportlich inaktiv, das sind 2,89mal so viele wie in der deutschen Vergleichsgruppe. Bei den Jungen mit

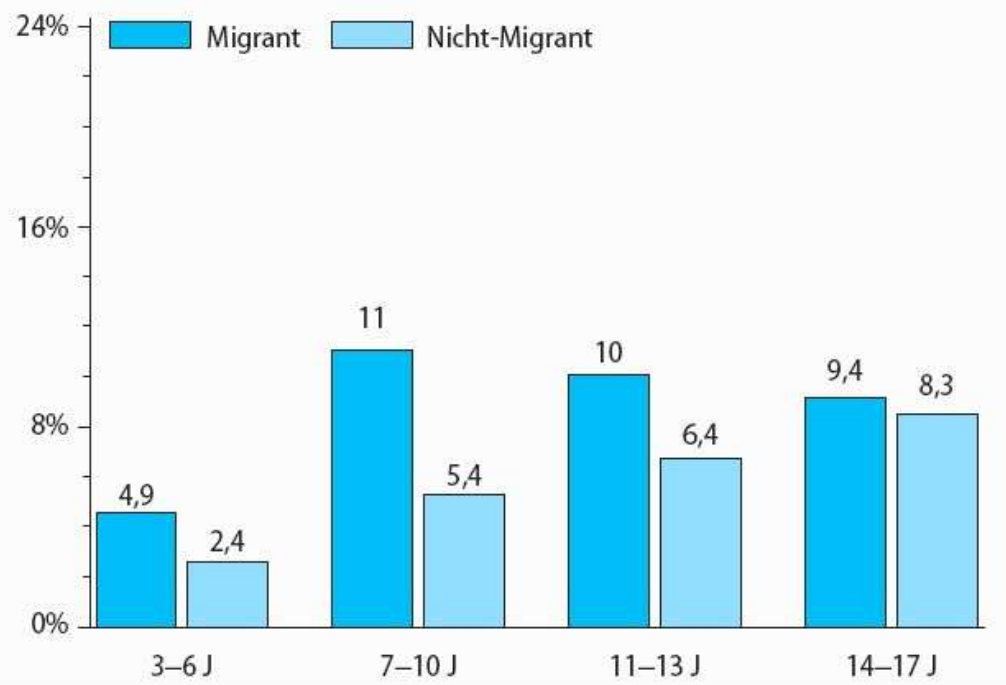
Migrationshintergrund sind es $1,7 \mathrm{mal}$ so viele wie in der Vergleichsgruppe [Lampert et al. 2007a].

Oft gehören Migrantenfamilien gleichzeitig der niedrigen sozialen Schicht an und sind daher besonders gesundheitlich gefährdet [Kurth und Schaffrath-Rosario 2007].

Abbildung 13: Prävalenz von Adipositas nach Migrationshintergrund in der KiGGS- Studie [Kurth und Schaffrath-Rosario 2007, S.740]

\subsubsection{Intellektuelle Fähigkeiten}

Besonders junge Kinder mit mentaler Retardierung oder eingeschränkten intellektuellen Fähigkeiten sind häufiger von Übergewicht und Adipositas betroffen als ihre Altersgenossen. Emerson ermittelte 2009 für 3-5-jährige Kinder mit Entwicklungsverzögerung eine OddsRatio von 1,8 für Adipositas. Unter den 6-7 Jahre alten Adipösen wiesen 23\% eine intellektuelle Beeinträchtigung auf [Emerson und Robertson 2010]. 


\subsection{Folgeerkrankungen}

\subsubsection{Das Metabolische Syndrom}

Für Kinder und Jugendliche gibt es keine international gültige Definition für das metabolische Syndrom, je nach Definition variieren daher die Angaben zur Prävalenz in den verschiedenen Studien. Ein Zusammenhang zwischen Adipositas und dem metabolischen Syndrom ist dennoch unbestreitbar [Saland 2007]. Eine mögliche Definition wird in Tabelle 5 dargestellt.

\begin{tabular}{|l|l|}
\hline Kriterium & Definition \\
\hline Adipositas & BMI $>$ 97. Perzentile \\
\hline Insulinresistenz & $\begin{array}{l}\text { nüchtern Glukose }>6,1 \mathrm{mmol} / \mathrm{I} \\
\text { HOMA-IR }>4,5 \\
\text { Pathologischer OGTT }\end{array}$ \\
\hline Hypertriglyceridämie & nüchtern Triglyceride $>$ 95. Perzentil \\
\hline $\begin{array}{l}\text { Niedriges HDL- } \\
\text { Cholesterin }\end{array}$ & nüchtern $\mathrm{HDL}<5$. Perzentil \\
\hline Arterielle Hypertonie & $\begin{array}{l}\text { Systolischer und/oder Diastolischer Wert }> \\
\text { 95.Perzentil nach Alter, Geschlecht und } \\
\text { Körpergröße in 3 Einzelmessungen }\end{array}$ \\
\hline
\end{tabular}

Tabelle 5: Definition des metabolischen Syndroms [Janner et al. 20 06, S.494]

Treffen drei oder mehr Kriterien zu, kann die Diagnose „metabolisches Syndrom“ gestellt werden [Janner et al. 2006]. Die Kriterien dieser Definition entsprechen der weit verbreiteten Definition der Internatinal Diabetes Federation (IDF) für Erwachsene [IDF 2006].

Die Arbeitsgemeinschaft Adipositas im Kindes- und Jugendalter nennt als weitere fakultative Kriterien die Hyperurikämie, das Syndrom der polyzystischen Ovarien (PCO Syndrom) und die Steatosis hepatis, die nicht-alkoholische Fettleberkrankheit (NAFLK) [AGA 2006].

In anderen Definitionen wird zum Beispiel auch der Hüftumfang als Kriterium für die viszerale Fettmasse herangezogen [Saland 2007].

Der gemeinsame Nenner all dieser Kriterien ist die Insulinresistenz, weshalb das metabolische Syndrom auch als Insulin Resistance Syndrome (IRS) bezeichnet wird [Janner et al. 2006]. Insulinresistenz entsteht durch Down-Regulation der Insulinrezeptoren bei dauerhaft bestehendem Hyperinsulinismus [Keller KM 2004] und hat eine starke genetische Komponente [Reinehr 2005].

Als klinischer Hinweis gilt Acanthosis nigricans an Axillen, Nacken und Bauchfalten (Abb.14) [Wabisch 2006]. Als Screening kann der so genannte HOMA-IR (Homöostase-ModellAssessment der Insulinresistenz) berechnet werden [AGA 2006], für den sich alters- und geschlechtsabhängige Normwerte zeigen [Allard et al. 2003].

Ein genaueres Ergebnis würde der Euglycaemic Clamp Test liefern, der jedoch sehr invasiv, teuer und zeitaufwändig und somit im klinischen Alltag nicht praktikabel ist [Saland 2007]. 


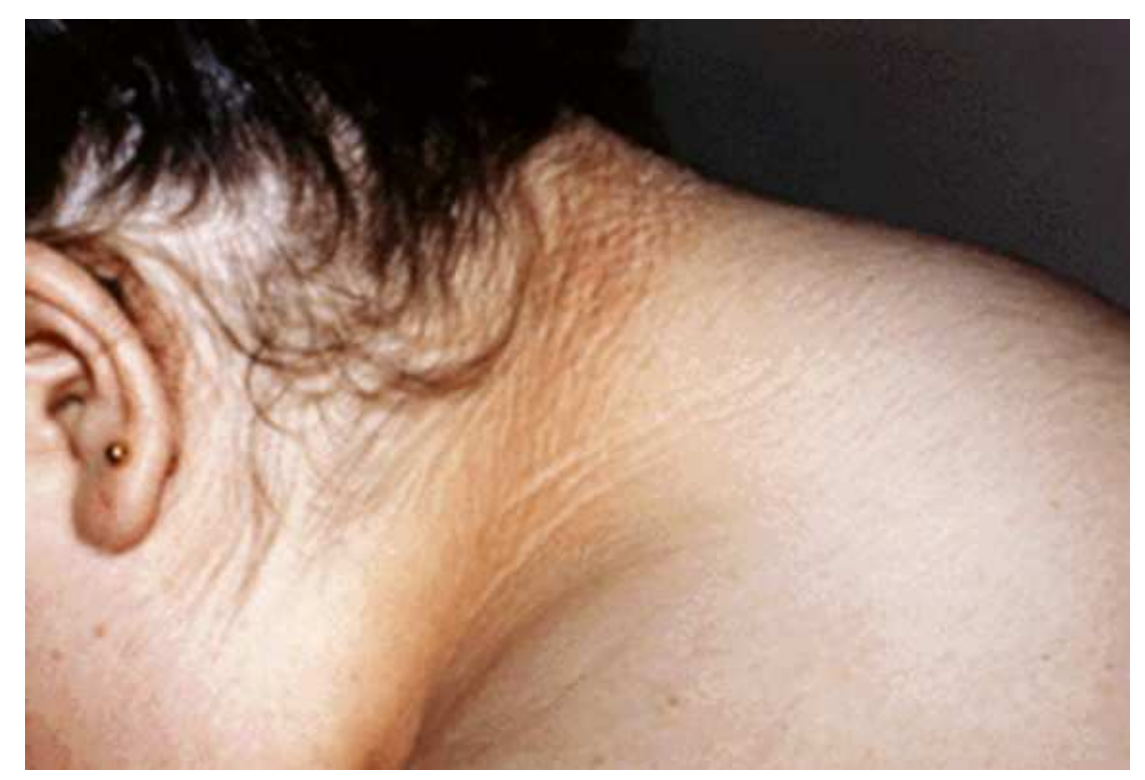

Abbildung 14: Acanthosis nigricans im Nacken [Ceders-Sinai 2010]

\subsubsection{Störungen der Glukosetoleranz}

Störungen der Glukosetoleranz treten vor der Pubertät sehr selten auf. Während der Pubertät kommt es jedoch zu einer physiologischen Erhöhung der Insulinresistenz [Reinehr 2005]. Etwa 3\% der adipösen Jugendlichen haben eine gestörte Glukosetoleranz, etwa 1\% leiden an einem manifesten Diabetes mellitus Typ 2 [Wabisch 2006].

Das Intervall zwischen den Schritten Insulinresistenz, der pathologischen Glukosetoleranz und dem manifesten Diabetes mellitus Typ 2 ist sehr variabel und hängt eher von der familiären Belastung als dem Ausmaß der Adipositas ab [Janner et al. 2006].

Ein oraler Glukosetoleranztest (OGTT) sollte bei Jugendlichen über zehn Jahren mit einem $\mathrm{BMI}>$ 90. Perzentil und zusätzlichen Risikofaktoren wie Zeichen einer Insulinresistenz (arterieller Hypertonus, Dyslipidämie, erhöhte Transaminasen, polyzystische Ovarien oder Acanthosis nigricans), Verwandten ersten oder zweiten Grades mit Diabetes mellitus Typ 2 sowie bei extrem adipösen Jugendlichen durchgeführt werden [AGA 2006].

\subsubsection{Steatosis hepatis}

Steatosis hepatis, auch die nicht-alkoholische Fettleberkrankheit (NAFLK), ist die häufigste Leberveränderung bei Kindern zwischen zwei und 19 Jahren [Schwimmer et al. 2009] und kommt bei Übergewichtigen mit einer Häufigkeit von 22 - 53\% vor [Keller KM 2004].

Es besteht eine enge Assoziation mit Insulinresistenz und dem metabolischem Syndrom [Wabisch 2006]. Auch der Leptinwert korreliert mit dem Ausmaß der Steatose (Verfettung) [Nobili et al. 2006]. 
Man geht von einer Two-Hit-Theorie auf dem Weg von der Steatose über die Steatohepatitis mit zentrolobulären Infiltraten, Nekrosen und Fibrosen bis hin zur Leberzirrhose aus [Keller KM 2004]. First Hit: durch erhöhte Fettsäurekonzentration im Plasma und Hyperinsulinismus (Hemmung der Abgabe von Chylomikronen) kommt es zur vermehrten Speicherung von Fettsäuren in den Hepatozyten [Keller KM 2004]. Die Steatose zeichnet sich sonographisch durch eine homogen erhöhte Echogenität des Leberparenchyms aus [Wabisch 2006]. Second Hit: durch Lipidperoxidation entsteht oxidativer Stress. So kommt es lokal zu Entzündungen, Apoptose und Fibrogenese. Im Labor sind ALT und AST auf ein 2-3-Faches der Norm erhöht, die Werte korrelieren mit Alter und BMI [Keller KM 2004]. Eine Steatose geht mit einer 20-prozentigen Wahrscheinlichkeit in eine Zirrhose über [Wiegand et al. 2010]. Eine definitive Diagnose kann jedoch nur bioptisch gestellt werden [Keller KM 2004]. Die Prognose bei Kindern ist nicht bekannt, bei Erwachsenen war im Stadium der Steatose eine vollständige Rückbildung durch die Modifikation von Lifestylefaktoren möglich, die Steatohepatitis und Leberzirrhose haben eine deutlich schlechtere Prognose [Keller KM 2004].

\subsubsection{Dyslipoproteinämie}

Die Fettstoffwechselstörung ist eine der häufigsten Störungen aus dem Cluster des metabolischen Syndroms bei Kinder und Jugendlichen und betrifft rund ein Viertel der Patienten [Reinehr 2005, Wabisch 2006].

Bei Kindern und Jugendlichen dominiert der Typ Ilb nach Fredriksen, der sich durch erniedrigtes HDL und erhöhte Triglyceridkonzentration im Serum auszeichnet [Wabisch 2006].

Eine pharmakologische Therapie wird bei Kindern und Jugendlichen selten notwendig: das HDL-Cholesterin lässt sich durch körperliche Aktivität und die Triglyceride durch verminderte Aufnahme von Mono- und Disacchariden positiv beeinflussen [AGA 2006].

Eine Erhöhung des LDL-Cholesterins tritt meist familiär gehäuft auf [Herold 2007]. Auch hier ist Therapie der Wahl eine Anpassung der Ernährungsgewohnheiten [AGA 2006].

Für die Validität der Laborergebnisse muss ein zwölfstündiges nahrungsfreies Intervall vor der Blutentnahme eingehalten werden, deswegen können die Lipoproteine bei Säuglingen und kleinen Kleinkindern nicht akkurat bestimmt werden [Wabisch 2006].

\subsubsection{Arterielle Hypertonie}

Bei einmaliger Messung beträgt die Inzidenz von arterieller Hypertonie bei adipösen Kindern und Jugendlichen etwa ein Drittel [Reinehr 2005]. Zum großen Teil lässt sich diese hohe 
Inzidenz auf den so genannten Weißkitteleffekt zurückzuführen. Dieser ist definiert als ein mittlerer systolischer Blutdruck (systolic blood pressure, SBP) oberhalb des 95. Perzentils in der Arztpraxis bei unauffälliger 24-Stunden-Blutdruckmessung [Urbina et al. 2008].

Vor der Messung des Blutdruckes muss ausgeschlossen werden, dass das Kind stimulierende Medikamente oder Lebensmittel zu sich genommen und die Harnblase entleert hat. Es sollte mindestens 5 Minuten in einem separaten Zimmer ruhig mit angelehntem Rücken und auf dem Boden abgestellten Füßen gesessen haben [NHBPEP 2004].

Außerdem ist die Wahl der Manschettengröße bei Kindern von besonderer Wichtigkeit. Die Manschette sollte $80 \%$ der Oberarmlänge bedecken [AGA 2006] und auf der Hälfte der Strecke zwischen Olecranon und Acromion angelegt werden [NHBPEP 2004].

Besonders bei Adipösen führt eine zu klein gewählte Manschette zu falsch hohen Ergebnissen. Im Gegensatz dazu kommt es durch zu groß gewählte Manschetten eher selten zum Unterschätzen der tatsächlichen Blutdruckwerte [NHBPEP 2004].

\begin{tabular}{|c|c|c|c|}
\hline Alter & $\begin{array}{l}\text { Breite } \\
\mathrm{cm}\end{array}$ & $\begin{array}{l}\text { Länge } \\
\mathrm{cm}\end{array}$ & $\begin{array}{l}\text { Oberarm } \\
\text { Umfang, } \mathrm{cm}^{*}\end{array}$ \\
\hline Neugeborenes & 4 & 8 & 10 \\
\hline Kleinkind & 6 & 12 & 15 \\
\hline Kind & 9 & 18 & 22 \\
\hline Jugendlicher & 10 & 24 & 26 \\
\hline Erwachsener & 13 & 30 & 34 \\
\hline Erwachsener, groß & 16 & 38 & 44 \\
\hline Oberschenkel & 20 & 42 & 52 \\
\hline
\end{tabular}

* Berechnet derart, dass die Manschette auch einen großen Oberarm mindestens bis zu $80 \%$ bedeckt

Tabelle 6: Richtwerte für die Manschettengröße zur Blutdruckmessung bei Kindern und Jugendlichen [AGA 2006, S.56]

Jeder oszillometrisch gemessene Wert oberhalb des 90. Perzentils sollte auskultatorisch nachgemessen werden [AGA 2006, Nguyen und Mitsnefes 2007, NHBPEP 2004].

Ausschlaggebend für die Diagnose einer arteriellen Hypertonie ist jedoch die Langzeitblutdruckmessung, die die Blutdruckwerte des Kindes in seiner normalen Umgebung und bei seinen gewohnten Tätigkeiten erfasst [Reinehr 2005, Wabisch 2006, Saland 2007], was im Bezug auf den Vorhersagewert des kardiovaskulären Risikos als überlegen betrachtet wird [Urbina et al. 2008].

Die arterielle Hypertonie ist bei adipösen Kindern und Jugendlichen öfter über-, bei Normalgewichten jedoch öfter unterdiagnostiziert [Saland 2007].

Für die Diagnosestellung werden alters-, geschlechts- und körpergrößenadaptierte Perzentilen herangezogen [AGA 2006]. Liegt bei Mehrfachmessung an mindestens drei verschiedenen Tagen der systolische und bzw. oder diastolische Mittelwert oberhalb des 95. 
Perzentils, liegt eine arterielle Hypertonie vor [AGA 06, NHBPEP 04, Sitzmann 2007, S.378]. Von Prähypertension spricht man, wenn in drei separaten Messungen der mittlere SBP oder diastolische Blutdruck (diastolic blood pressure, DBP) zwischen dem 90. und 95. Perzentil liegen [Nguyen und Mitsnefes 2007, NHBPEP 2004].

Im vierten Bericht zum National Health and Nutrition Examination Survey (NHANES) wurde die kindliche Hypertonie erstmals in Stadien eingeteilt [Nguyen und Mitsnefes 2007].

\begin{tabular}{|l|l|l|}
\hline \multicolumn{2}{|l|}{ Stadium } & Konsequenz \\
\hline 90.-95. Perzentil & Prähypertension & Beginnen von Lifestylemodifikation \\
\hline 95.-99.Perzentil $(+5 \mathrm{mmHg})$ & Stadium I & $\begin{array}{l}\text { Lifestylemodifikation und Kontolle nach } \\
\text { spätestens zwei Wochen }\end{array}$ \\
\hline >99.Perzentil $(+5 \mathrm{mmHg})$ & Stadium II & $\begin{array}{l}\text { Überweisung zum Kindernephrologen } \\
\text { innerhalb einer Woche }\end{array}$ \\
\hline
\end{tabular}

Tabelle 7: Klassifizierung der arteriellen Hypertonie im Kindes- und Jugendalter [nach Nguyen und Mitsnefes 2007]

Es gibt zwei Formen der Hypertonie, die nach Ätiologie unterschieden werden, die essenzielle (primäre) und die sekundäre Hypertonie, wobei die sekundäre Hypertonie bei Kindern die weitaus häufigere Form ist [Sitzmann 2007, S.378f].

Deshalb sollten folgende Ursachen für eine sekundäre Hypertonie klinisch (Flushing, Palpitationen, Ödeme, Makrohämaturie), laborchemisch (Nierenwerte, Blutbild, ReninAldosteron-Quotient, Urinanalyse und -kultur, Katecholamine im Urin) und bildgebend (Nierensonographie) ausgeschlossen werden:

\begin{tabular}{|l|l|}
\hline Organsystem & Ursache \\
\hline Kardiovaskulär & Aortenisthmusstenose \\
& Entzündliche Veränderungen von \\
& Geäßen \\
\hline Renal & Nierenarterien-Stenose \\
& Fibromuskuläre Dysplasie \\
& Hämolytisch-urämisches Syndrom \\
& Glomerulonephritis \\
\hline Systemisch/ & Phäochromozytom \\
Endokrinologisch & M.Cushing/ Cushing-Syndrom \\
& Primärer Hyperaldosteronismus \\
& Hyperthyreose \\
& Androgenitales Syndrom \\
& Systemischer Lupus erythematodes \\
\hline
\end{tabular}

Tabelle 8: Ursachen einer sekundären arteriellen Hypertonie im Kindes- und Jugendalter [Sitzmann 2007, S.379]

Ein weiterer diagnostischer Hinweis auf eine sekundäre Hypertonie ist ein Fehlen des nächtlichen Blutdruckabfalls im 24-Stunden-Blutdruckprofil [Herold 2007]. 
Die essenzielle Hypertonie tritt familiär gehäuft auf [Sitzmann 2007, S.378]. Begünstigende Faktoren sind neben Adipositas [Sitzmann 2007, S.378] auch ein niedriges Geburtsgewicht und das Schlaf-Apnoe-Syndrom, bei dem auch eine nächtliche Hypertonie auftritt [Nguyen und Mitsnefes 2007].

Lifestylemodifikation gilt als der primäre Therapieansatz [AGA 2006]. Einige Autoren empfehlen jedoch bei manifesten Endorganschäden eine konsequente medikamentöse Therapie [Nguyen und Mitsnefes 2007]. Der häufigste Hinweis auf Endorganschaden bei Kindern und Jugendlichen ist die Linksherzhypertrophie im Verhältnis zur Körperlänge [Nguyen und Mitsnefes 2007], aber auch vaskuläre Veränderungen kommen im jungen Alter bereits vor [Lande et al. 2006]. Es wird ein Zusammenhang zwischen der Mediadicke der A. carotis und dem Anteil der erhöhten Werte in der 24-Stunden-Blutdruckmessung festgestellt, was auf eine Dosis-Wirkbeziehung schließen lässt [Lande et al. 2006].

\subsubsection{Hyperurikämie}

Von einer Hyperurikämie sind etwa ein 20\% aller übergewichtigen Kinder und Jugendlichen betroffen [Reinehr 2005].

In mehreren Studien konnte ein konzentrationsunabhängiger Zusammenhang zwischen Hyperurikämie und arterieller Hypertonie festgestellt werden [Johnoson et al. 2005].

Durch erhöhte Harnsäurekonzentration kommt es in den Nieren zum Endothelschaden und zur Vasokonstriktion, wodurch das Renin-Angiotensin-System aktiviert wird, was wiederum eine Ursache für arterielle Hypertonie ist [Johnoson et al. 2005].

Weiterhin lagern sich Harnsäurekristalle in die glatten Muskelzellen der Tunica media der Gefäße ein, was zur Proliferation dieser Zellen mit lokaler Entzündungsreaktion führt und gleichzeitig mit einer Aktivierung des RAS einhergeht [Johnoson et al. 2005].

Feig und Johnson untersuchten 125 hypertone Kinder zwischen sechs und 18 Jahren.

Sie fanden über $5,5 \mathrm{mg} / \mathrm{dl}$ erhöhte Harnsäurewerte bei $89 \%$ der Kinder mit essenzieller Hypertonie und bei 30\% der Kinder mit sekundärer Hypertonie.

Bei keinem der Kinder mit Weißkittelhypertonie und aus der Vergleichsgruppe waren die Harnsäurespiegel erhöht [Feig und Johnson 2003].

Eine Normalisierung der Harnsäure, alimentär oder medikamentös, sollte somit als Bestandteil einer antihypertensiven Behandlung angesehen [Johnson 05, Alper 05] oder sogar präventiv, zur Vermeidung arterieller Hypertonie, angestrebt werden [Alper et al. 2005].

In einer Studie mit 78 hypertonen Kindern zwischen acht und 13 Jahren wurde nach Senkung der Serumharnsäure eine lineare Korrelation zwischen Blutdruck und Harnsäure 
festgestellt: eine Senkung der Harnsäurekonzentration um $1 \mathrm{mg} / \mathrm{dl}$ führte zu einer Senkung des SBP um $3 \mathrm{mmHg}$ [Franco et al. 2006].

In einer anderen Studie konnte man den Blutdruck durch in Behandlung mit Allopurinol in vier von fünf Fällen senken [Nguyen und Mitsnefes 2007].

\subsubsection{Weitere kardiovaskuläre Risikofaktoren}

\subsubsection{Lipoprotein(a)}

Eine Besonderheit unter den Lipoproteinen stellt das Makromolekül Lipoprotein(a) (Lp(a)) dar. Es setzt sich aus LDL-Cholesterin und Apolipoprotein A zusammen [Utermann 1989] und besitzt so zum einem strukturelle Eigenschaften von Lipoproteinen, zum anderen thrombolysehemmende Eigenschaften, die durch die strukturelle Ähnlichkeit von Apolipoprotein A und Fibrinogen zustande kommen [Kraft 06]. Durch diese Ähnlichkeit kann das $L p(a)$ das Fibrinogenmolekül aus seiner Bindung verdrängen und trägt so in besonderem Maße zur Bildung artherogener Plaques bei [Kraft 06].

Die Blutkonzentration kann stark variieren und ist genetische determiniert [Utermann 1989, Kraft 06].

Nach dem sechsten Lebensmonat zeigt die Serumkonzentration von $L p(a)$ keinen Altersgang, daher kann der Normwert für Erwachsene $(<30 \mathrm{mg} / \mathrm{dl})$ auch bei Kindern und Jugendlichen angewendet werden [Wabisch 2006].

\subsubsection{Hyperhomozysteinämie}

Homozystein ist ein Zwischenprodukt im Abbau der schwefelhaltigen Aminosäure Methionin und wird, auf Grund seiner vielfältigen artherogenen Effekte und hohen Zytotoxizität, für etwa 10\% des gesamten kardiovaskulären Risikos verantwortlich gemacht [Fowler 2005].

Eine Senkung der Plasmakonzentration um $3.5 \mu \mathrm{mol} / \mathrm{l}$ kann das Gesamtrisiko für kardiovaskuläre Ereignisse um 25\% senken [Stanger et al. 2003].

Genetische Prädisposition, Folsäure-, Vitamin B12- und Vitamin B6-Mangel sowie Lifestylefaktoren können die Plasmakonzentration beeinflussen [Fowler 2005].

Verantwortlich ist meist der Folsäuremangel, der häufigste Vitaminmangel in Europa. Die wichtigste Quelle des extrem umweltlabilen Vitamins sind frisches Obst und Gemüse. Mit $300 \mu \mathrm{g}$ bleibt die durchschnittliche Aufnahme in Deutschland, Österreich und der Schweiz

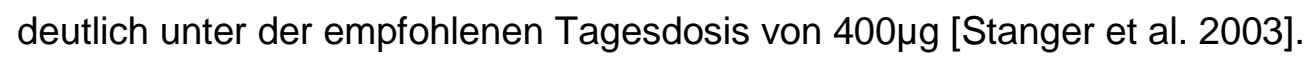


Bei Patienten mit einem ohnehin erhöhten kardiovaskulären Risiko kann eine Senkung des Homozysteinspiegels durch Vitaminsubstitution erreicht werden, mit Beendigung der Substitution wird die Plasmakonzentration erneut ansteigen [Stanger et al. 2003].

\begin{tabular}{|l|l|l|}
\hline Konzentration $[\mu \mathrm{mol} / \mathrm{l}]$ & Stadium & wahrscheinliche Ursache \\
\hline $12-30$ & leicht erhöht & Vitaminmangel, Schilddrüsendysfunktion \\
\hline $30-100$ & mittelschwer & Enzymdefekt, chronisches Nierenversagen \\
\hline$>100$ & schwer & $\begin{array}{l}\text { bei schwerwiegenden angeborenen } \\
\text { Störungen }\end{array}$ \\
\hline
\end{tabular}

Tabelle 9: Mögliche Ursachen einer erhöhten Homozystein-Serum-Konzentration [Stanger et al. 2003, S.1401]

\subsubsection{Syndrom der polyzystischen Ovarien (PCOS)}

Vom PCOS, auch Stein-Leventhal-Syndrom [Bühling und Friedmann 2004], sind etwa 10\% der Frauen im reproduktionsfähigen Alter betroffen [Wertheim et al. 2007].

Es ist mit Adipositas [Bühling und Friedmann 2004] und metabolischem Syndrom [AGA 2006] assoziiert.

Für die Diagnose eines PCOS müssen zwei von drei der Rotterdam-Kriterien zutreffen:

1. Anovolation, Oligomenorrhoe

2. Hyperandrogenismus (Hirsutismus, Klitorishypertrophie, tiefe Stimme, Alopezie, Mammahypotrophie, Seborrhoe und Akne [Bühling und Friedmann 2004])

3. sonographisch polyzystische Ovarien (2-5fach vergrößert [Bühling und Friedmann 2004])

[Wertheim et al. 2007]

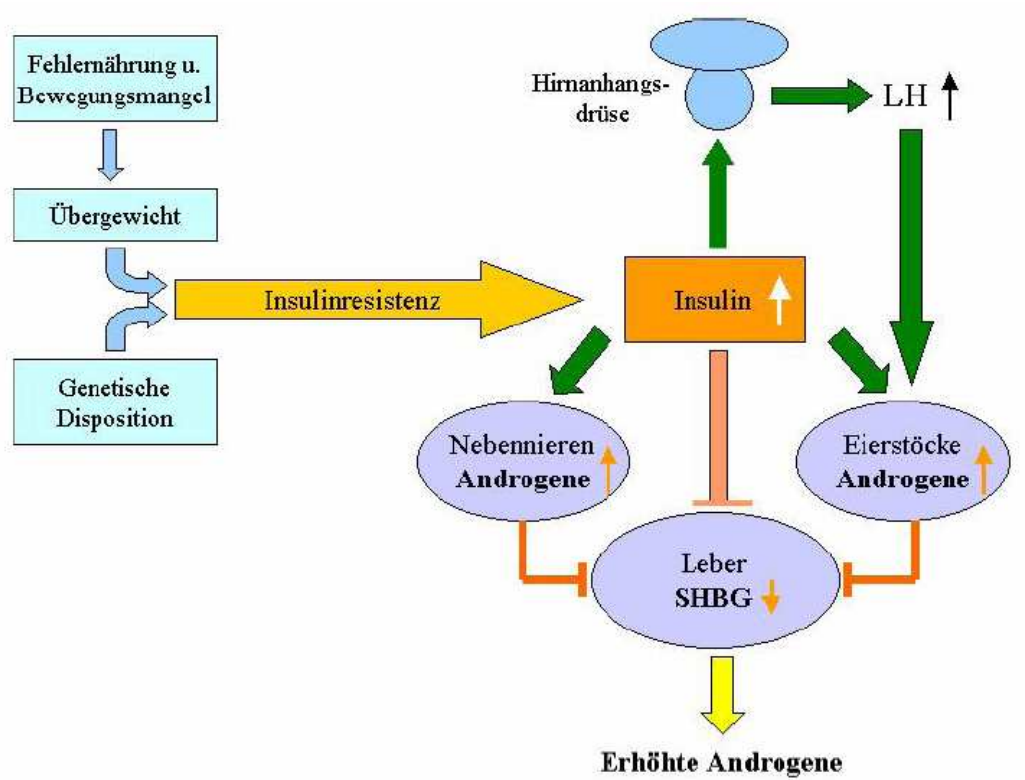

Abbildung 15: Zusammenhang zwischen Insulinresistenz und PCOS [Hinney 2008] 
Der durch Insulinresistenz hervorgerufene Hyperinsulinismus bewirkt direkt an Ovarien und Nebennieren eine gesteigerte Androgensynthese, zudem wird an der Leber die Bildung von SHBG (Sexualhormonbindendes Globulin) gehemmt, was zu einer zusätzlichen Erhöhung der freien und somit biologisch wirksamen Androgene führt.

\subsubsection{Störungen der Pubertätsentwicklung}

Durch die Expression der P450-Androgenase im Fettgewebe, die Androgene in Östrogene umwandelt, kann es in der Pubertät bei Jungen und Mädchen zu Abweichungen der normalen Entwicklung kommen [Fischer-Posovszky und Wabisch 2004], die im Ausmaß der Energiereserve des Körpers korreliert [Wabisch 2006].

Generell haben adipöse Kinder eine frühe Adrenarche und ein akzeleriertes Knochenalter mit vorübergehend erhöhter Wachstumsgeschwindigkeit [Wabisch 2006].

Eine frühe Reifeentwicklung ist allgemein mit einem erhöhten Adipositasrisiko im Erwachsenenalter assoziiert [Wabisch 2006].

Bei Jungen hat eine erhöhte Östrogenkonzentration eine Feminisierung zur Folge [FischerPosovszky und Wabisch 2004], die sich in Form einer Pseudogynäkomastie und eines Pseudohypogenitalismus äußert [Wabisch 2006]. Adipöse Mädchen haben durchschnittlich eine frühere Menarche [Wabisch 2006]. Somit kommt es bei Jungen durch den erhöhten Östrogeneinfluss zu einer Verzögerung, bei den Mädchen zu einer Beschleunigung der Pubertätsentwicklung.

\subsubsection{Adipositas-assoziierte Erkrankungen des Bewegungsapparates}

Kindliche Knochen sind besonders weich und biegsam, die Sehnen und Bänder haben ihre endgültige Zugfestigkeit noch nicht erreicht und das Knorpelgewebe der Wachstumsfugen ist besonders anfällig gegenüber übermäßigem Druck und Scherkräften [Dordel 2007]. Die größte Diskrepanz zwischen Belastbarkeit und Belastung besteht während des präpubertären Wachstumsschubs, besonders bei Übergewicht und Adipositas. Einseitige Belastung oder Fehl- und Überbelastung, wie zum Beispiel langes Sitzen, können zu Osteochondrosen und irreversiblen Haltungsschäden führen [Dordel 2007].

\subsubsection{Wirbelsäule}

Die aufrechte Haltung ist ein psychophysiologisches System, für dessen Funktion die Psyche, der aktive und der passive Bewegungsapparat eine Rolle spielen [Dordel 2007]. 
Bei adipösen Kindern und Jugendlichen bildet sich durch die vermehrte Fettmasse, also vermehrte Last, besonders am Bauch, oft ein Hohlrücken (Hyperlordose der LWS) und zur statischen Kompensation ein Rundrücken (Hyperkyphose der BWS) aus, was durch vermehrtes Sitzen und ein ungünstiges Selbstbild weiter gefördert wird [Dordel 2007].

Während des Wachstums reicht der Mineralisierungsgrad der Wirbelkörper nicht aus, um das erhöhte Körpergewicht zu tragen. Im Verhältnis zu ihrem Gewicht haben Adipöse noch weniger Knochenmaterial als ihre normalgewichtigen Altersgenossen, so dass Fehlbelastungen sich besonders stark auswirken [Wills 2004].

Etwa 17\% der adipösen Mädchen und 9\% der adipösen Jungen klagen über Rückenschmerzen [Dordel 2007], besonders im lumbosakralen Bereich der Wirbelsäule [Wills 2004].

\subsubsection{Beinachse}

Die Beinachse unterliegt einer physiologischen Entwicklung vom Genu varum im Säuglingsalter über ein Genu valgum nach dem Erlernen des freien Laufens und der Normalisierung der Beinachse, die im Alter von acht bis zehn Jahren abgeschlossen sein sollte. Außerdem finden noch eine Reihe komplexer Torsionsvorgänge statt [Dordel 2007]. Bei mechanischer Überbelastung durch Adipositas bleibt diese Normalisierung häufig aus, so dass das Bein in der X-Stellung bleibt [Dordel 2007], was die Entstehung einer Gonarthrose zusätzlich begünstigt [Wabisch 2006].

\subsubsection{Fußskelett}

Auch das Fußskelett macht eine physiologische Entwicklung durch. Säuglinge und Kleinkinder haben physiologischerweise einen Plattfuß. Mit dem Beginn des Laufens richtet sich die Ferse spontan in eine $10^{\circ}$ Valgusstellung a uf [Dordel 2007].

Mit zwei bis sechs Jahren bildet sich normalerweise das Fußgewölbe aus. Tut es das nicht, kann sich ein vorhandener Plattfuß auch noch im Verlauf der weiteren Entwicklung normalisieren [Dordel].

Man unterscheidet den häufigeren flexiblen vom rigiden Plattfuß. Beim flexiblen Plattfuß bildet sich im Zehenstand ein Fußgewölbe aus [Dordel 2007].

Das Missverhältnis zwischen Belastung und Belastbarkeit führt auch hier zu einer erhöhten Prävalenz bei Adipösen. Durch Bandschwäche und muskuläre Insuffizienz besteht die Gefahr der strukturellen Veränderungen, die zu einer Fixierung des Plattfußes führen [Dordel 2007]. 


\subsubsection{Epiphysiolysis capitis femoris}

Es handelt sich um eine Dislokation der Hüftkopfepiphyse, die vor allem während des präpubertären Wachstumschubes auftritt. Jungen sind dreimal so häufig betroffen wie Mädchen [Sitzmann 2007, S.849].

Zuerst kommt es zur Epiphysiolysis incipidens, einer Auflockerung der Epiphysenfuge. Bei Belastung löst sich die Epiphyse, Epiphysiolysis capitis femoris acuta, was wegen der großen Gefahr einer Hüftkopfnekrose eine sofortige operative Korrektur erfordert [Sitzmann 2007, S.849]. Eine Sonderstellung nimmt die so genannte Lenta-Form ein. Hier erfolgt ein langsames Abrutschen auf dem metaphysären Plateau nach hinten unten. Klinisch zeigt sich typischerweise eine schmerzhafte Fixierung des betroffenen Beins in Adduktion und Außenrotation [Sitzmann 2007, S.849].

Einen Risikofaktor für die Erkrankung stellt die Dystrophia adiposogenitalis dar [Dordel 2007, Sitzmann 2007, S.849], wozu eine Stammfettsucht und ein unterentwickeltes Genitale gehören. Das ist ein Hinweis darauf, dass nicht nur das Gewicht, sondern auch endokrine und metabolische Faktoren Einfluss nehmen [Dordel 2007], dennoch spielt die mechanische Komponente ätiologisch eine wichtige Rolle.

Laut Studienlage sind von den betroffenen Jugendlichen zwischen $51,5 \%$ und $72 \%$ adipös.

Bei adipösen Patienten kommt auch häufiger ein beidseitiger Verlauf vor [Wills 2004].

Als mögliche Ursache wird, neben der erhöhten vertikalen Belastung, eine vermehrte Hüftadduktion bei Adipösen diskutiert, die zur Entstehung starker Scherkräfte auf die empfindliche Wachstumsfuge führt [Wills 2004].

Der latente Verlauf der Epiphysiolysis capitis femoris wird als Tilt-Deformität bezeichnet [Dordel 2007, Wabisch 2006] und begünstigt in besonderem Maße die Entstehung einer Coxarthrose [Wabisch 2006].

\subsubsection{Blount Disease}

Blount Disease ist eine aseptische Knochennekrose [Dordel 2007], die die mediale Seite der proximalen Tibiaepiphyse betrifft und zu einer Varusdeformität der Tibia führt [Wills 2004]. Etwa zwei Drittel der Patienten sind übergewichtig [Dordel 2007], zeigen meist auch einen schwereren Verlauf und müssen häufiger operiert werden als Normgewichtige [Wills 2004]. Man unterscheidet die infantile (1-3 Jahre) von der adoleszenten Form, die zu über 90\% adipöse, dunkelhäutige Jungen betrifft [Wills 2004].

Die infantile Form tritt auf dem Gipfel des kindlichen Genu varum und bei beiden Geschlechtern gleich häufig auf. Durch die Wirkung von Scherkräften auf die Wachstumsfuge [Dordel 2007] wird das Wachstum einseitig unterdrückt [Wills 2004]. 60- 
$80 \%$ der Kinder sind bilateral betroffen [Wills 2004]. Es kommt zur fixierten Varusdeformität mit Innentorsion des Unterschenkels [Dordel 2007]. Hierbei hat der Varus ein größeres präarthrotische Potenzial als der Valgus [Dordel 2007].

Die adoleszente Form hat aufgrund der geringeren Wachstumsgeschwindigkeit die bessere Prognose und tritt meist auch nur einseitig auf [Dordel 2007].

\subsubsection{Frakturen und Traumata}

Adipositas ist ein eigenständiger Risikofaktor für Frakturen. Adipöse Kinder und Jugendliche stürzen ungeschickter und mit mehr Wucht [Wills 2004]. Die meisten Frakturen ereignen sich zur Zeit des präpubertären Wachstumsschubes, der, zusätzlich zur verminderten Knochendichte bei Bewegungsmangel, mit einem verminderten Mineralisierungsgrad der Knochen im Verhältnis zur Körperlänge einhergeht [Wills 2004]. Auch Zerrungen treten bei Adipösen häufig auf [Wabisch 2006].

\subsubsection{Schlaf-Apnoe-Syndrom}

Beim obstruktiven Schnarchen, dem Schlaf-Apnoe-Syndrom (SAS), kommt es zu nächtlicher Hypoventilation und konsekutiven Hypoxämien [Wabisch 2006].

Aus der Hypoxämie resultiert eine Aufwachreaktion (Arousal) mit vertiefter Atmung. Der REM- und Tiefschlafanteil ist vermindert [Herold 2007].

Als unmittelbare Folge des Schlafdefizits treten Tagschläfrigkeit mit Sekundenschlaf, Konzentrations- und Gedächtnisstörungen, depressive Verstimmungen und morgendliche Kopfschmerzen und Mundtrockenheit auf [Herold 2007].

Als Risikofaktor gilt neben Adipositas die bei Kindern physiologische Hyperplasie der Adenoide. Dies spielt besonders bei Kindern im Alter von zwei bis sechs Jahren atiologisch weitaus häufiger eine Rolle. Im jugendlichen Alter überwiegt, wie bei Erwachsenen, die Adipositas als Ursache [Kaditis et al. 2008]. Kinder mit Hyperplasie der Adenoide sind typischerweise untergewichtig und klein, durch eine Adenektomie kommt es bei den meisten zu einer Zunahme von Körpergewicht, Körpergröße und BMI [Soultan et al. 1999].

Die Rezidivrate des SAS war bei Adipösen in einer Studie 3,7-mal höher als bei normalgewichtigen Kindern [Kaditis et al. 2008]. In einer anderen Studie konnten nur 37\% der Patienten durch Adenotomie geheilt werden, während 53\% auch nach dem Eingriff weiterhin auf C-PAP Beatmung (Continuous Positive Airway Pressure) angewiesen waren [Shine et al. 2006]. Außerdem wurde postoperativ auch bei adipösen und extrem adipösen Kindern eine weitere Zunahme an Körpergewicht beobachtet [Soultan et al. 1999]. 


\begin{tabular}{|l|l|}
\hline \multicolumn{2}{|l|}{ Gemeinsame Co-Morbiditäten von Adipositas und SAS: } \\
\hline Verhalten & $\begin{array}{l}\text { geringes Selbstvertrauen } \\
\text { ADHS, Verminderte Impulskontrolle }\end{array}$ \\
\hline Stoffwechsel & $\begin{array}{l}\text { Insulinresistenz } \\
\text { Pathologische Glukosetoleranz }\end{array}$ \\
\hline Immunsystem & $\begin{array}{l}\text { Erhöhte Entzündungsparameter } \\
\text { durch oxidativen Stress bei } \\
\text { chronisch-intermittierenden } \\
\text { Hypoxämien }\end{array}$ \\
\hline Herz-Kreislauf & $\begin{array}{l}\text { arterielle Hypertonie, } \\
\text { Linksherzhypertrophie }\end{array}$ \\
\hline
\end{tabular}

Tabelle 10: Gemeinsame Co-Morbiditäten von SAS und Adipositas [levers-Landis und Redline 2007, S.438]

\subsubsection{Psychiatrische Co-Morbiditäten}

Eine Befragung adipöser Kinder und Jugendlicher zur Lebensqualität ergab ein mit krebskranken Kindern vergleichbares Ergebnis [Warschburger 2006].

Adipositas ist eine sozial unerwünschte, für jeden sichtbare Krankheit, die Stigmatisierung und Ablehnung hervorruft. Das Körpergewicht wird als kontrollierbar und die Adipösen somit als selbstverantwortlich betrachtet [Warschburger 2006].

Über $40 \%$ der adipösen Kinder und Jugendlichen leiden unter Depressionen, etwa 40\% unter Angststörungen, 15\% unter Somatisierungsstörungen und 17\% unter Essstörungen [Reinehr 2005], dabei treten oft mehrere Erkrankungen co-morbide in einem Patienten auf [Britz et al. 2000].

Bei einer Untersuchung von 51 stationären Patienten der auf Langzeittherapie von Adipositas bei Kindern und Jugendlichen spezialisierten Klinik INSULA in Berchtesgarden, die mindestens $50 \%$ oberhalb des Durchschnittsgewichtes lagen, hatten nur $29,8 \%$ gar keine psychiatrische Erkrankung, 25,3\% erhielten eine Diagnose, 10,5\% zwei Diagnosen und 34\% drei oder mehr psychiatrische Diagnosen [Britz et al. 2000]. Besonders häufig traten affektive Störungen zusammen mit Angststörungen auf, hiervon waren etwa $30 \%$ der Patienten betroffen [Britz et al. 2000].

Eine große Rolle für ein negatives Selbstkonzept und Selbstwertgefühl von Kindern und Jugendlichen spielt die Unzufriedenheit der Eltern über das Gewicht des Kindes [Warschburger 2006].

Bei gemeinsamen Auftreten von Adipositas und Depressionen kommt ein Teufelskreis zustande: Adipositas beeinträchtigt das Selbstwertgefühl und führt so zu Depressionen, die sich wiederum negativ auf den Antrieb und die sozialen Kontakte auswirken. Der verminderte Energieverbrauch bewirkt dann eine weitere Gewichtszunahme [Warschburger 2006]. 


\subsection{Fragestellung}

Ziel dieser Doktorarbeit ist es, herauszufinden, wie sich das Kollektiv der in der Adipositassprechstunde des Sozialpädiatrischen Zentrums Göttingen behandelten Patienten in psychosozialer Hinsicht, im Bezug auf das Geschlecht, Alter und das Ausmaß des Übergewichtes oder der Adipositas zusammensetzt. Bearbeitet wurden die folgenden Fragestellungen: Können Risikofaktoren für die Entstehung der Adipositas identifiziert werden? Wie stark ist ihr jeweiliger Einfluss? Welche Folgeerkrankungen treten bereits im Kindesalter auf? Welche körperlichen und psychosozialen Faktoren bestimmen den Therapieerfolg? Diese Fragen werden ausführlich erläutert. 


\section{Material/ Methoden}

Die Daten von 462 übergewichtigen oder adipösen Kindern und Jugendlichen, die sich im Rahmen der Adipositassprechstunde des Sozialpädiatrischen Zentrums (SPZ) Göttingen im Zeitraum vom 01.03.2005 bis 29.02.2008 vorstellten, wurden retrospektiv untersucht. Hiervon waren 219 (47,4\%) Mädchen und 243 (52,6\%) Jungen im Alter von drei Monaten bis zu 18 Jahren.

\subsection{Datenerfassung und -auswertung}

Die Erfassung der Daten erfolgte standardmäßig durch das von der Universität Ulm in Zusammenarbeit mit der Arbeitsgemeinschaft Adipositas im Kindes- und Jugendalter (AGA) entwickelte Adipositas-Patienten-Verlaufs-Programm (APV), einem auf elektronischer Datenverarbeitung basierendem Dokumentationsprogramm für adipöse Kinder und Jugendliche [Reinehr et al. 2008]. Dieses Programm wird von 125 Zentren in Deutschland, Österreich und der Schweiz eingesetzt und dient neben einer standardisierten, prospektiven Verlaufsdokumentation auch der Qualitätssicherung. Die für die Adipositastherapie relevanten Parameter wurden anhand der AGA-Leitlinien ausgewählt [AGA 2006].

Die statistische Auswertung der Daten erfolgte mit Statistica Version 9 und Microsoft Exel 2002. Signifikanz wurde für einen $p$-Wert unter 0,05 angenommen.

Nach Bildung verschiedener Gruppen, zum Beispiel nach Alter, Co-Morbidität oder sozialen Kriterien, wurde für die jeweilige Gruppe Median, Maximal- und Minimalwert und das obere und untere Quartil für den Ausgangswert des BMI-SDS oder die Änderung des BMI-SDS nach einer definierten Therapiedauer ermittelt und die Verteilung der Werte im Boxplot dargestellt. Durchschnitt, Standardabweichung und $\mathrm{p}$-Wert wurden meist tabellarisch angegeben. Für die lineare Regression verwendeten wir ebenfalls Statistica 9.0, während die polynomische Regression mit Microsoft Exel 2002 erfolgte.

\subsection{Status bei Erstvorstellung}

\subsubsection{Biometrische Daten}

Bei Erstvorstellung wurden Gewicht und Größe der Kinder und Jugendlichen gemessen und daraus der Body-Mass-Index errechnet (Formel: BMI= Körpergewicht [kg]/ (Körperlänge $[\mathrm{m}])^{2}$ ). Anhand der Perzentilenkurven nach Kromeyer-Hauschild [AGA 2006] ermittelten wir das jeweilige Perzentil. 
Ein weiteres Mittel zur Quantifizierung des BMI, vor allem zur Verlaufskontrolle bei Kindern und Jugendlichen, die sich im Wachstum befinden, aber auch besonders zur Quantifizierung der extremen Adipositas (>99,5. Perzentil), ist die LMS-Methode [Cole et al. 1990]. Ein BMISDS (Standard Deviation Score) entspricht der Standardabweichung von der fünfzigsten Perzentile für Alter und Geschlecht und berechnet sich wie folgt:

$$
\operatorname{SDS}_{\mathrm{LMS}}=\frac{[B M I / M(t)]^{L(t)}-1}{L(t) S(t)}
$$

Der BMI ist hier der einzige Individualwert des Kindes bzw. Jugendlichen, die restlichen Variablen entsprechen alters- und geschlechtsspezifischen Konstanten.

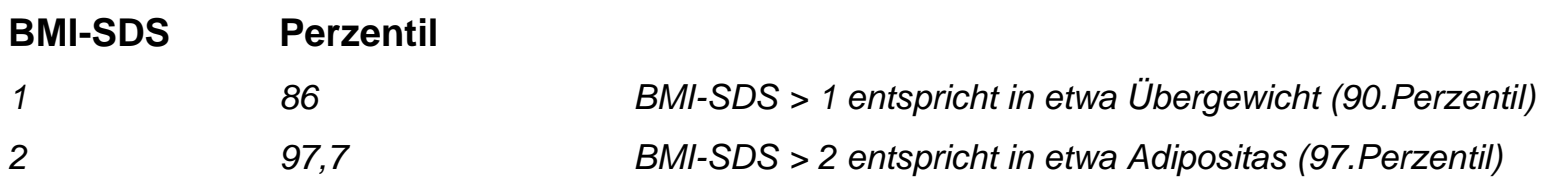

[AGA 2006]

Von den 462 Kindern und Jugendlichen liegen Verlaufsdaten für den BMI-SDS nach sechs Monaten von 151 Kindern vor. Dabei muss beachtet werden, dass sich 43 Kinder und Jugendliche erstmals nach dem 01.09.2007 vorstellten, also weniger als sechs Monate vor Schluss der Datenaufnahme. Nach einem Jahr existieren Daten von 91 Kindern und Jugendlichen, wobei 124 sich nach dem 01.03.2007, also weniger als ein Jahr vor Studienabschluss, vorstellten.

Nach sechs Monaten waren damit noch mindestens 36,04\% in Therapie, nach einem Jahr noch $26,92 \%$.

\subsubsection{Anamnese}

Die psychosozialen Faktoren wie Familiensituation, Anzahl der Geschwister, Migrationshintergrund, Ausbildung und Berufstätigkeit der Eltern, Kinder und Jugendlichen sowie die biometrischen Daten der Eltern wurden anhand eines standardisierten Anamnesebogens im Rahmen der ärztlichen Vorstellung erfragt.

Ein Kind mit Migrationshintergrund wird dabei definiert als Kind, das selbst, oder dessen Eltern, aus einem anderen Land nach Deutschland eingewandert sind. Der Begriff ist unabhängig von der Staatsbürgerschaft [Schenk 2010]. 


\subsubsection{Begleiterkrankungen}

Bei allen Kindern und Jugendlichen wurde bei Erstvorstellung ein ausführlicher körperlicher Untersuchungsbefund erhoben. Anhand der Leitlinien der Arbeitsgemeinschaft Adipositas im Kindes- und Jugendalter zum Ausschluss organischer Ursachen, zur Erfassung von Risikofaktoren und zur Diagnostik von Folgeerkrankungen erfolgte die Bestimmung eines klinischen Labors [AGA 2006] am nüchternen Patienten. Hatte der Patient bereits gegessen, wurde die Probenentnahme am folgenden Morgen nach Einhaltung einer ausreichenden Nüchterperiode von mindestens zwölf Stunden vorgenommen.

\subsubsection{Metabolisches Syndrom}

\subsection{Insulinresistenz und Störungen der Glukosetoleranz}

Der HOMA-IR (Homöostase-Modell-Assessment der Insulinresistenz) dient als Maß für die Insulinresistenz (Formel: HOMA-IR= Nüchtern-Serum-Insulin [mIU/l] Nüchtern-Glukose [mmol/l]/22,5) [AGA 2006].

Das Insulin wurde in Doppelantikörper-/PEG-Technik bestimmt (Insulin RIA DSL-1600 von DSL), die Bestimmung der Glukose erfolgte via enzymatischem Farbtest im venösen Plasma. In der Literatur findet man für Kinder und Jugendliche unterschiedliche Referenzwerte, wir benutzten die altersspezifischen Perzentile nach Allard et al. (2003):

\begin{tabular}{|c|c|c|c|c|c|c|}
\hline \multicolumn{7}{|l|}{ Jungen } \\
\cline { 1 - 6 } Alter & 5. Perzentil & 25. Perzentil & 50. Perzentil & 75. Perzentil & 95. Perzentil & Mittelwert \\
\hline 9 & 0,33 & 0,55 & 0,83 & 1,15 & 1,88 & 0,95 \\
\hline 13 & 0,58 & 0,91 & 1,4 & 2,04 & 3,28 & 1,66 \\
\hline 16 & 0,61 & 0,94 & 1,28 & 1,69 & 3,31 & 1,55 \\
\hline
\end{tabular}

\begin{tabular}{|c|c|c|c|c|c|c|}
\hline Alter & 5. Perzentil & 25. Perzentil & 50. Perzentil & 75. Perzentil & 95. Perzentil & Mittelwert \\
\hline 9 & 0,34 & 0,63 & 0,9 & 1,29 & 2,07 & 1,13 \\
\hline 13 & 0,79 & 1,25 & 1,62 & 2,27 & 3,86 & 1,9 \\
\hline 16 & 0,68 & 1,03 & 1,42 & 2,01 & 3,1 & 1,8 \\
\hline
\end{tabular}

Tabelle 11: HOMA-IR Referenzwerte [Allard et al. 2003, S. 646]

Bei Erstvorstellung erfolgte bei jedem Patienten ein Nüchternglukose-Screening aus dem venösen Plasma. Eine gestörte Glukosetoleranz wurde ab $100 \mathrm{mg} / \mathrm{dl}$ angenommen, ein Diabetes mellitus ab 126 mg/dl. 
Einen oralen Glukosetoleranztest (OGTT) führten wir bei Patienten oberhalb des 90. Perzentils über 10 Jahren durch, wenn zusätzlich einer oder mehrere der folgenden Risikofaktoren vorlagen:

1. Typ 2- Diabetes mellitus bei Verwandten 1.-2. Grades

2. Extreme Adipositas (BM I>P.99,5)

3. Zeichen der Insulinresistenz oder mit ihr assoziierter Veränderungen (arterieller

4. Hypertonus, Dyslipidämie, erhöhte Transaminasen, Polyzystisches Ovarsyndrom,

5. Acanthosis nigricans

6. Zugehörigkeit zu einer ethnischen Gruppe mit erhöhtem Risiko (z.B. Asiaten, Afrikaner)

[AGA 2006]

Nach drei Tagen kohlehydratreicher Ernährung folgt eine Fastenperiode über Nacht von mindestens zehn Stunden. Danach erhielten die Kinder 1,75 g Glukose/ kg Körpergewicht, bis zu maximal $75 \mathrm{~g}$ Glukose als orale Belastung. Es erfolgten Blutentnahmen nüchtern und nach 30, 60, 90 und 120 Minuten mit Glukosebestimmung im venösen Plasma. Als Grenze zur gestörten Glukosetoleranz wurden $140 \mathrm{mg} / \mathrm{dl}$, zum Diabestes mellitus $200 \mathrm{mg} / \mathrm{dl}$ für den Zweistundenwert angenommen [AGA 06, Wabisch 2006].

\subsection{Steatosis hepatis}

Zur Bestimmung der Transaminasen wurde ein optimierter UV-Test nach IFCC bei $37^{\circ} \mathrm{C}$ durchgeführt (AST: Rolf Greiner BioChemica/ ALT: DiaSys Diagnostic Systems).

Als Grenzwerte legten wir für AST und ALT jeweils 50 U/l fest [Wiegand et al. 2010].

Der Ausschluss einer anders geartete Lebererkrankung als Steatosis hepatis erfolgte anamnestisch und klinisch.

\subsection{Dyslipoproteinämie und Triglyceridämie}

Die Grenzwerte für Fettstoffwechelstörungen wurden wie folgt festgelegt:

\begin{tabular}{|c|c|c|c|c|c|c|}
\hline Lipide & Methode & & 1-3Jahre & 4-7 Jahre & 8-15 Jahre & $\begin{array}{l}16-19 \\
\text { Jahre }\end{array}$ \\
\hline Cholsesterin & \multirow[t]{3}{*}{ enzymatischer Farbtest } & $\begin{array}{l}\text { Cholesterin CHOD-PAP } \\
\text { Cobas (Roche) }\end{array}$ & $<140 \mathrm{mg} / \mathrm{dl}$ & $<150 \mathrm{mg} / \mathrm{dl}$ & $<160 \mathrm{mg} / \mathrm{dl}$ & $<170 \mathrm{mg} / \mathrm{dl}$ \\
\hline LDL & & Rolf Greiner BioChemica & $<90 \mathrm{mg} / \mathrm{dl}$ & $<100 \mathrm{mg} / \mathrm{dl}$ & $<110 \mathrm{mg} / \mathrm{dl}$ & $<110 \mathrm{mg} / \mathrm{dl}$ \\
\hline $\mathrm{HDL}$ & & HLD-C L-Type (Wako) & $>35 \mathrm{mg} / \mathrm{dl}$ & $>40 \mathrm{mg} / \mathrm{dl}$ & $>40 \mathrm{mg} / \mathrm{dl}$ & $>40 \mathrm{mg} / \mathrm{dl}$ \\
\hline TG & $\begin{array}{l}\text { enzymatischer Farbtest } \\
\text { nach Wahlefeld }\end{array}$ & $\begin{array}{l}\text { Tryglyceride GPO-PAP } \\
\text { (Roche) }\end{array}$ & $<100 \mathrm{mg} / \mathrm{dl}$ & $<110 \mathrm{mg} / \mathrm{dl}$ & $<110 \mathrm{mg} / \mathrm{dl}$ & $<120 \mathrm{mg} / \mathrm{dl}$ \\
\hline
\end{tabular}

Tabelle 12: Methoden zur Bestimmung der Serumlipide, Grenzwerte [Wabisch 2006, S.89] 


\subsection{Arterielle Hypertonie}

Bei allen Patienten wurde routinemäßig bei jeder Vorstellung nach einer Ruhezeit von mindestens fünf Minuten und nach Entleerung der Harnblase der Blutdruck gemessen. Zeigten sich hierbei Auffälligkeiten, führte man die Kinder, zum Ausschluss einer manifesten arteriellen Hypertonie, einer ambulante 24-Stunden-Blutdruckmessung zu. Die Interpretation erfolgte anhand der Leitlinien der American Heart Association:

\begin{tabular}{|l|l|l|}
\hline & Mean ambulatory SBP & SBP load [\%] \\
\hline Normaler Blutdruck & $<95$. Perzentil & $<25$ \\
\hline Arterielle Hypertonie & $>95$. Perzentil & $25-50$ \\
\hline Schwere art. Hypertonie & $>95$. Perzentil & $>50$ \\
\hline
\end{tabular}

Tabelle 13: Definition der arteriellen Hypertonie bei Kindern und Jugendlichen in der Langzeitblutdruckmessung [Urbina et al. 2008, S. 437]

Als mean ambulatory SBP bezeichnet den mittleren systolischen Druck aus allen Werten in 24 Stunden, unter SBP load versteht man den Anteil an systolischen Werten aus 24 Stunden, der sich oberhalb der Norm befand [Urbina et al. 2008].

Die Festlegung dieser Werte erfolgte nach geschlechts- und körpergrößenspezifischer Perzentile. Bei keinem der Kinder oder Jugendlichen lag klinisch oder laborchemisch der Verdacht auf eine sekundäre Hypertonie vor.

\subsubsection{5. Übersicht über die Komponenten des metabolischen Syndroms}

Die Definition wurde anhand der Kriterien nach Janner et al. mit o.g. Referenzwerten festgelegt:

\begin{tabular}{|l|l|}
\hline Kriterium & Definition \\
\hline Adipositas & BMI-SDS $>2$ \\
\hline Arterielle Hypertonie & $\begin{array}{l}\text { in 24-Stunden-Messung nach AHA mit } \\
\text { körperlängenspezifischen Perzentilen }\end{array}$ \\
\hline Insulinresistenz oder & $\begin{array}{l}\text { HOMA-IR }>95 . \text { Perzentil nach Allard et al. oder } \\
\text { Nüchtern-Glukose } \geq 100 \mathrm{mg} / \mathrm{dl} \text { im venösen } \\
\text { Plasma } \\
\text { Gestörte Glukosetoleranz } \\
\end{array}$ \\
$\begin{array}{l}\text { Zweistunden-Wert } \geq 140 \mathrm{mg} / \mathrm{dl} \text { im venösen } \\
\text { Plasma }\end{array}$ \\
\hline Vermindertes HDL & alterspezifische Normwerte siehe 2.2.3.1.3 \\
\hline Erhöhte Triglyceride & alterspezifische Normwerte siehe 2.2.3.1.3 \\
\hline
\end{tabular}

Tabelle 14: Festlegung der Kriterien zur Definition des metabolischen Syndroms 
Treffen drei oder mehr der o.g. Kriterien zu, so kann die Diagnose eines metabolischen Syndroms gestellt werden.

\subsubsection{Leptin}

Die Bestimmung von Leptin erfolgte mittels Radioimmunoassay (LINCO Research).

\subsubsection{Hyperurikämie}

Die Harnsäure wurde via enzymatischem Farbtest mit der ALDH-Methode (Rolf Greiner BioChemica) bestimmt. Als Referenzbereich galten 1,9-5,9 mg/dl.

\subsubsection{Kardiovaskuläre Risikofaktoren}

\subsection{Lipoprotein(a)}

Die Bestimmung der Lp(a) erfolgte anhand partikelverstärkter Immunturbidimetrie mit Cobas Integra 400/700/800 (Roche). Als geschlechts- und altersunabhängiger Grenzwert wurden $30 \mathrm{mg} / \mathrm{dl}$ angenommen. Ein erhöhter $\mathrm{Lp}(\mathrm{a})$-Spiegel kann auch im Rahmen von Nierenerkrankungen auftreten [Dieplinger und Kronenberg 1999]. Bei keinem der Kinder unseres Kollektivs lag hierfür ein klinischer Verdacht vor.

\subsection{Hyperhomozysteinämie}

Homozystein wurde anhand Floureszenzpolarisationsimmunoassay (FPIA, Abbott Axsym System) bestimmt. Als pathologisch galten Werte außerhalb des Bereiches 5-15 $\mu \mathrm{mol} / \mathrm{l}$.

\subsection{Verlauf der Gewichtsentwicklung vor der Intervention}

\subsubsection{Analyse der Daten aus den Vorsorgeuntersuchungen}

Die Daten der Vorsorgeuntersuchungen, Geburtsgewicht und -woche wurden anhand der gelben Kindervorsorgehefte erfasst. Für die Prüfung der Signifikanz führten wir hier mit Microsoft Exel 2002 den G-Test durch. 


\subsubsection{Regression des Gewichtsverlaufes vor der Intervention}

In die Regressionsanalyse gingen alle bekannten biometrischen Daten der Patienten ein, die vor dem Termin der Erstvorstellung im SPZ lagen.

Wir führten eine polynomische Regression sechsten Grades mit Microsoft Excel durch und berechneten anhand der sich daraus ergebenden Funktionen die Wendepunkte.

\subsection{Therapiegruppen}

Je nach Therapieaufwand wurden Gruppen wie folgt definiert:

\begin{tabular}{|l|l|}
\hline Therapiegruppe & Definition \\
\hline Diagnostik & $\begin{array}{l}\text { ein Arztbesuch mit jeweils maximal einem } \\
\text { Termin Ernährungsberatung und/ oder } \\
\text { Psychotherapie }\end{array}$ \\
\hline Einzelberatung & $\begin{array}{l}\text { mehrere Arztbesuche mit mehreren Terminen } \\
\text { Ernährungsberatung oder Psychotherapie mit } \\
\text { maximal einem Termin der jeweils anderen } \\
\text { Therapiemodalität }\end{array}$ \\
\hline Multiprofessionelles Team & $\begin{array}{l}\text { mehrere Arztbesuche mit insgesamt mehr als } \\
\text { fünf Therapieterminen, mit zwei oder mehr } \\
\text { Terminen jeder Therapiemodalität }\end{array}$ \\
\hline AGA & $\begin{array}{l}\text { Schulung nach den Leitlinien der } \\
\text { Arbeitsgemeinschaft Adipositas im Kindes- und } \\
\text { Jugendalter (AGA) }\end{array}$ \\
\hline
\end{tabular}

Tabelle 15: Einteilung der Therapiegruppen im SPZ Göttingen

Der Inhalt der einzelnen Therapiemodalitäten entsprach dabei den Leitlinien der AGA. Die Schulung nach AGA-Leitlinien ist die einzige Therapieform, die nach einem standardisierten Konzept und nicht individuell erfolgte.

\subsubsection{Beurteilung des Therapieerfolges}

Zur Bestimmung des Therapieerfolges wurde die Differenz des BMI-SDS bei Erstvorstellung und nach sechs und zwölf Monaten, jeweils \pm einem Monat berechnet. Es erfolgt die Einteilung der Gruppen nach Differenz des BMI-SDS (keine Reduktion, Reduktion bis 0,2, Reduktion 0,2-0,5, Reduktion > 0,5). Nach der AGA ist eine BMI-SDS Reduktion um mehr als 0,5 über ein Jahr als besonders relevanter Therapieerfolg definiert. In Studien ergab sich ab einer Reduktion des BMI-SDS in diesem Bereich messbare Zeichen für eine Verminderung des kardiovaskulären Risikos [Reinehr et al. 2009]. 


\subsubsection{Psychosoziale Einflüsse auf den Therapieerfolg}

Um den Therapieerfolg in Abhängigkeit der psychosozialen Situation auszuwerten, bildeten wir soziale Risikogruppen. Die Gruppen setzten sich wie folgt zusammen: Familienstruktur, Anzahl der Geschwister, Kinder und Jugendliche mit Migrationshintergrund, BMI der Eltern, Ausbildung und Beruf der Eltern und Schulbildung der Patienten.

Die Gruppenbildung und Auswertung nach psychosozialen Faktoren erfolgten analog zu der Auswertung bei Erstvorstellung. 


\section{Ergebnis}

\subsection{Status bei Erstvorstellung (EV)}

Ein Aufnahmestatus wurde in der Adipositasambulanz des Sozialpädiatrischen Zentrums der Universitätsmedizin Göttingen im betrachteten Zeitraum von 462 Kindern und Jugendlichen erhoben. Hiervon waren 219 (47,4\%) Mädchen und 243 (52,6\%) Jungen.

Bei keinem der Patienten ergab sich ein Hinweis auf ein adipogenes Syndrom oder eine monogenetische Ursache für die Adipositas.

\subsubsection{Biometrische Daten}

Bei Erstvorstellung betrug das mittlere Alter bei Mädchen 10,53 $( \pm 3,95)$ Jahre bei einem BMI-SDS von durchschnittlich 2,66 $( \pm 0,75)$, bei den Jungen wurde ein Durchschnittsalter von 11,07 $( \pm 3,13)$ Jahren bei einem mittleren BMI-SDS von 2,45 $( \pm 0,59)$ ermittelt. Hierbei war der Unterschied im BMI-SDS zwischen Jungen und Mädchen $(p=0,0012)$, nicht aber der Altersunterschied signifikant.

Bezogen auf den BMI-SDS war die am stärksten vertretene Gruppe bei den Jungen die adipösen Kinder und Jugendlichen mit einem BMI zwischen dem 97. und 99,5. Perzentil mit 53,5\% und bei den Mädchen die extrem adipösen oberhalb des 99,5. Perzentils mit 48,86\%.

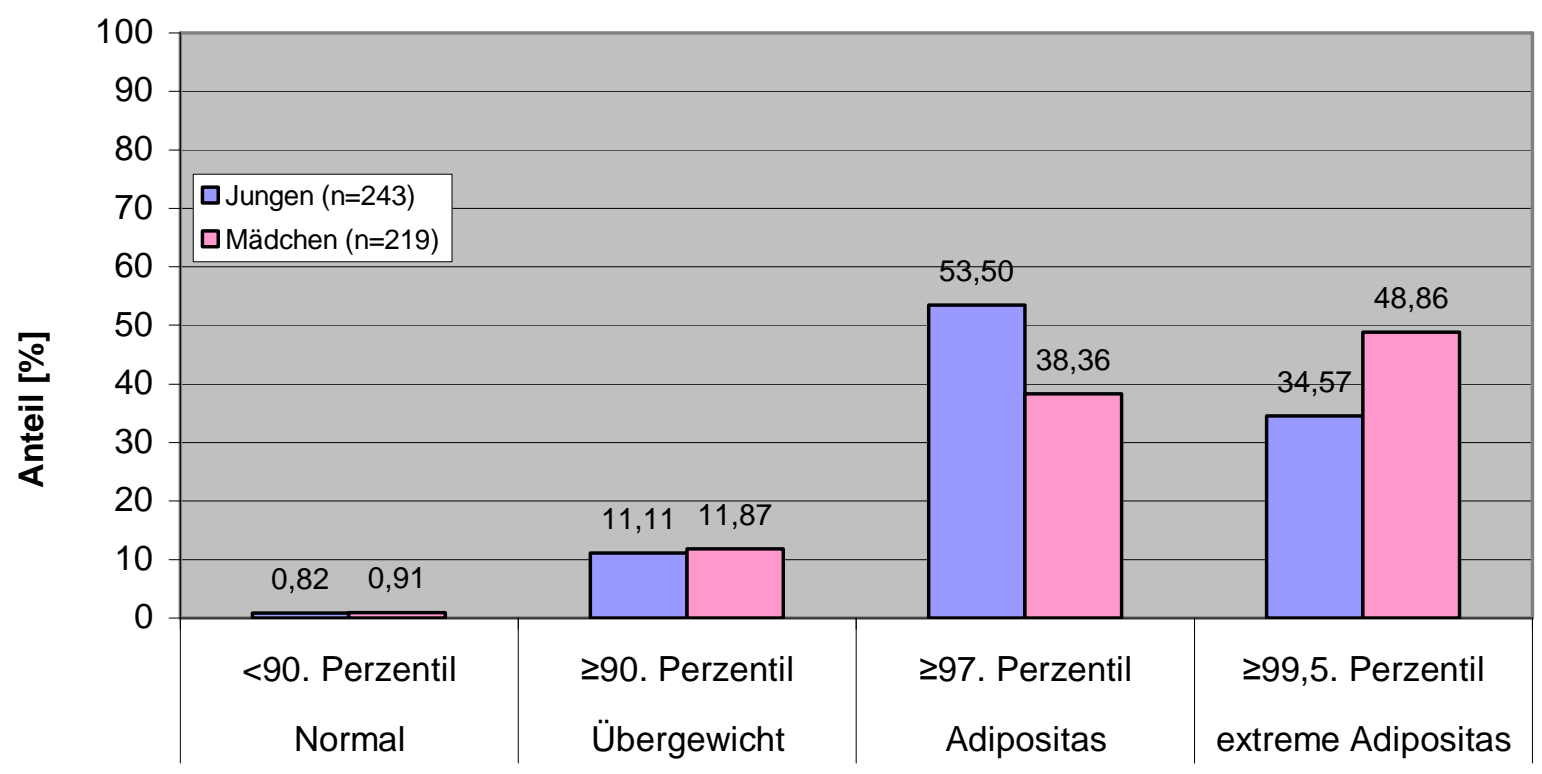

Abbildung 16: Verteilung des BMI bei Erstvorstellung

Die am häufigsten vertretene Altersgruppe bei der Erstvorstellung, besonders bei den Jungen, waren die Patienten zwischen 11 und 14 Jahren. Der zweitgrößte Anteil stellte sich 
im Alter zwischen 7 und 10 Jahren vor. In den Gruppen 4-6 und 15-18 Jahre sind die Mädchen etwa doppelt so stark vertreten wie die Jungen.

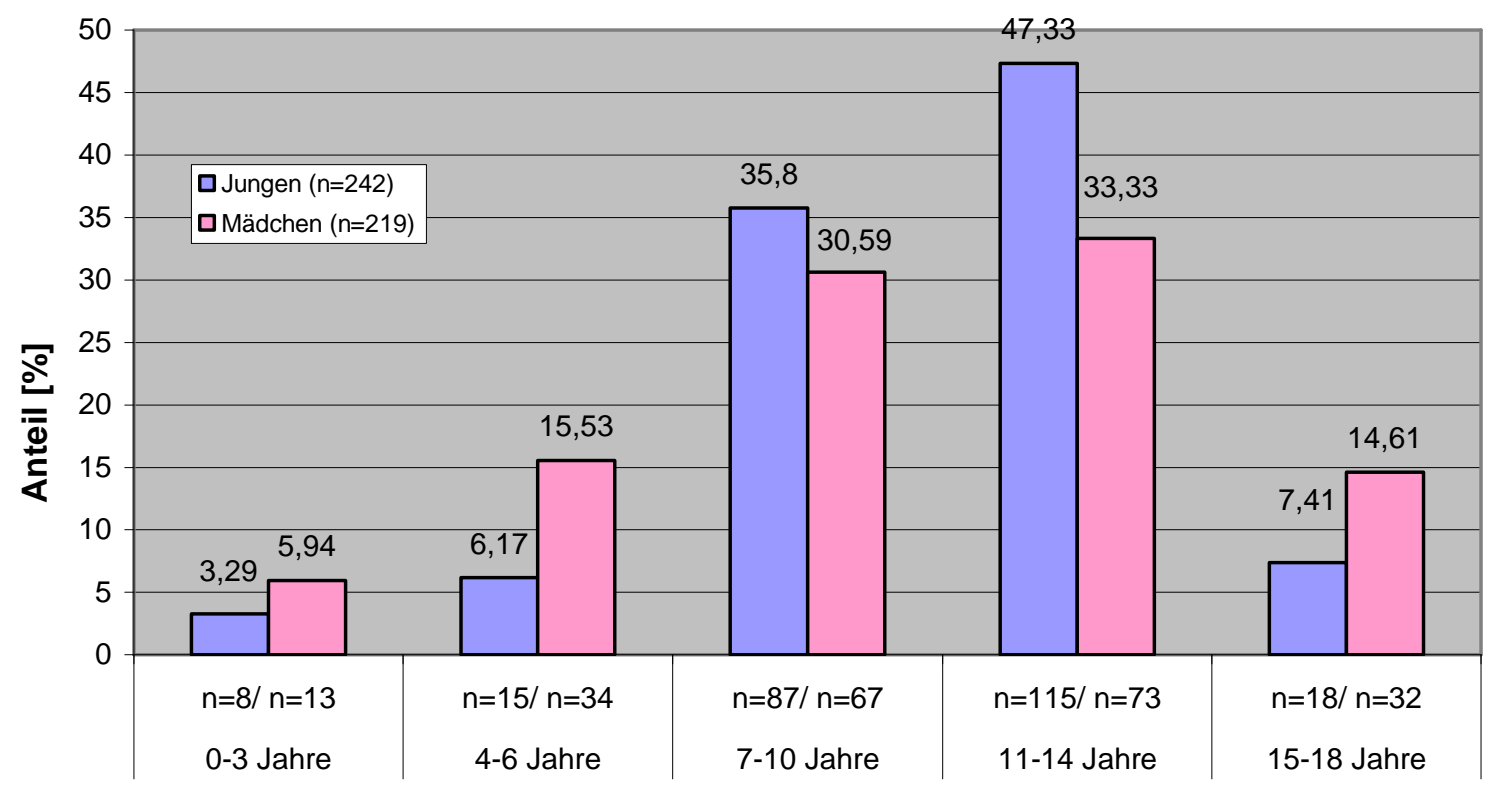

Abbildung 17: Alter bei Erstvorstellung

Stellt man die Verteilung der BMI-SDS-Werte in den einzelnen Altersgruppen dar, so zeigt sich, dass die am häufigsten vertretenen Altersgruppen zwischen sieben und 14 Jahren im Mittel am wenigsten stark von Adipositas betroffen sind. Klein- und Vorschulkinder hingegen haben im Mittel einen signifikant höheren BMI-SDS. Stärker betroffen als die Kinder zwischen sieben und zehn Jahren sind auch die älteren Teenager, dieser Unterschied ist allerdings nur bei den Mädchen signifikant.

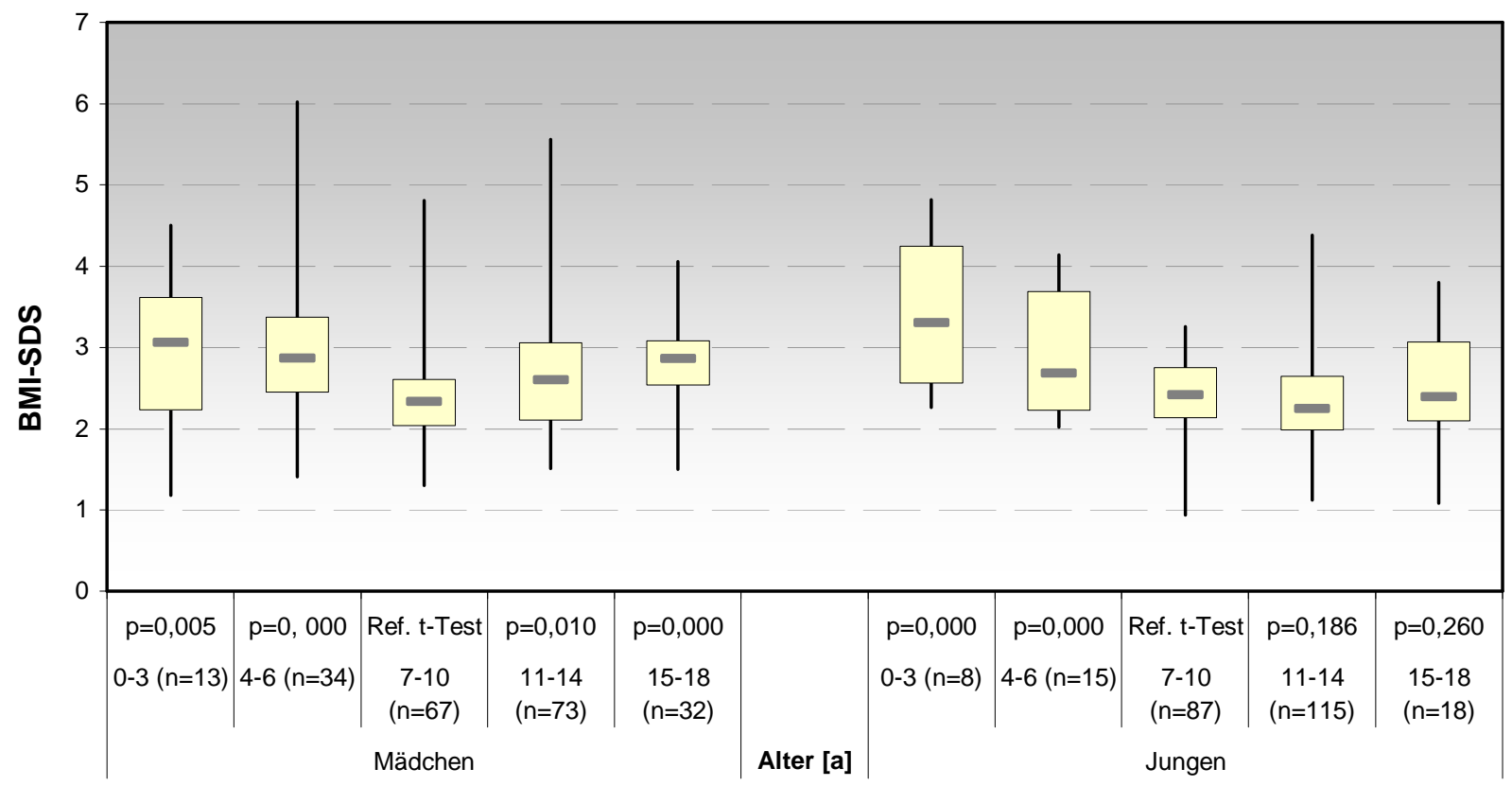

Abbildung 18: BMI-SDS nach Geschlecht und Altersgruppen bei Erstvorstellung 


\subsubsection{Psychosoziale Faktoren}

\subsubsection{Charakterisierung der Familien}

Von 344 Kindern und Jugendlichen wurde die familiäre Situation erfasst. Der mit Abstand größte Anteil (72,09\%) lebt in einer klassischen Familienstruktur mit beiden leiblichen Eltern. Die anderen 27,91\% leben mit einem allein erziehenden Elternteil, in einer Stiefelternfamilie, bei Adoptiv- oder Pflegeeltern oder sind im Heim untergebracht. Den zweitgrößten Anteil nehmen mit 19,48\% die Kinder und Jugendlichen mit allein erziehendem Elternteil ein.

Ein signifikanter Unterschied in der Ausprägung der Adipositas zeigte sich für keine der Gruppen ( $p=0,82$ bei Kindern und Jugendlichen aus klassischer Familie vs. denen aus Haushalten mit ohne klassische Familienstruktur).

Die Anzahl der Geschwister konnte von 390 Patienten ermittelt werden. Die meisten leben in Familien mit einem (45,38\%) oder zwei (22,05\%) Geschwisterkindern, 20,00\% sind Einzelkinder und $12,56 \%$ haben drei oder mehr Geschwister. Es zeigte sich ein signifikanter Unterschied im BMI-SDS zwischen Kindern und Jugenlichen mit einem oder zwei Geschwisterkindern und sowohl Einzelkindern als auch den Patienten aus kinderreicheren Familien: bei Kindern mit einem oder zwei Geschwistern war die Adipositas geringer ausgeprägt als bei den anderen, wobei Kinder mit zwei Geschwistern noch etwas weniger betroffen waren (mittlerer BMI- SDS 2,36 $\pm 0,60$ ) als Kinder und Jugendliche mit nur einem Geschwisterkind (mittlerer BMISDS 2,51 $\pm 0,64$ ).

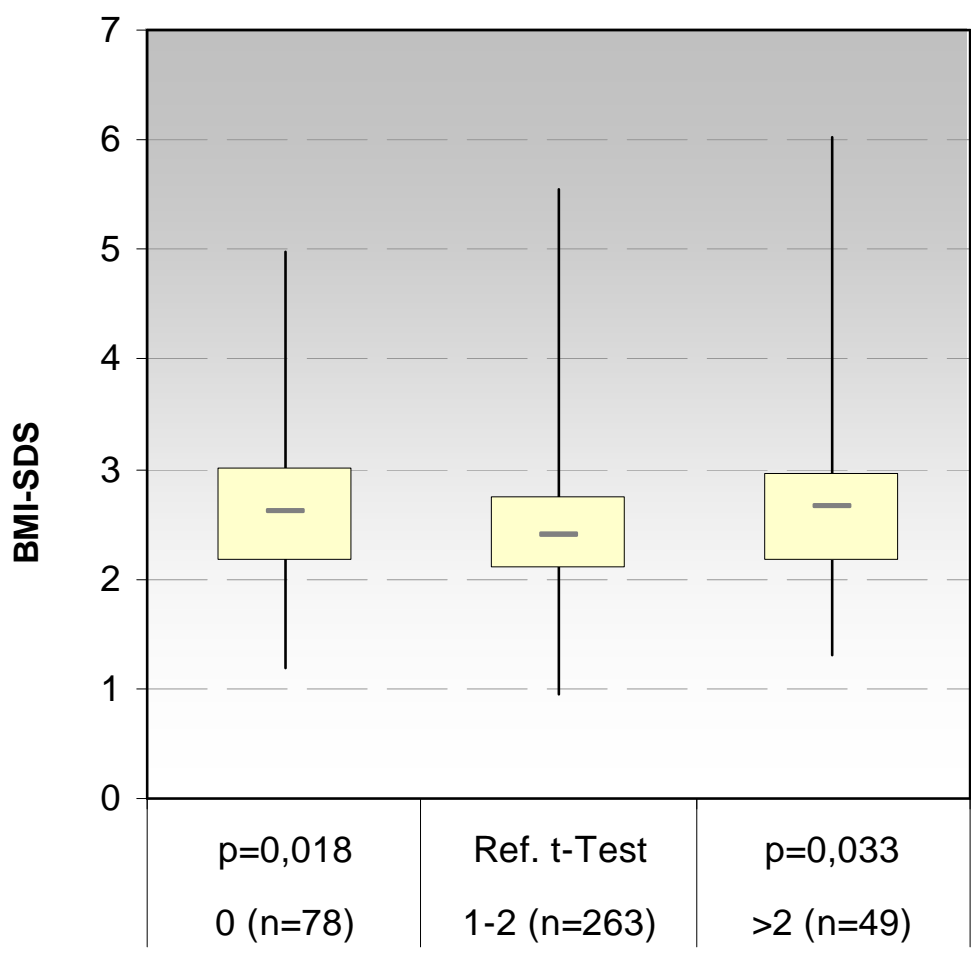

Anzahl der Geschwister 


\subsubsection{Migrationshintergrund}

Der Anteil an Kindern und Jugendlichen mit Migrationshintergrund beträgt insgesamt 15,70\%, wovon 12\% der Patienten zwei Elternteile und 3,7\% ein Elternteil mit ausländischer Herkunft haben.

Ein signifikanter Unterschied im BMI-SDS zwischen Kindern mit und ohne Migrationshintergrund konnte für unser Kollektiv nicht nachgewiesen werden ( $p=0,64$ für einen, $p=0,45$ für zwei Elternteile mit Migrationshintergrund).

\subsubsection{BMI der Eltern}

Betrachtet man den BMI der Eltern, so stellt man fest, dass nur $27,30 \%$ der Mütter und $21,57 \%$ der Väter normalgewichtig sind.

\begin{tabular}{|c|c|c|c|c|c|}
\hline & Untergewicht & Normal & Übergewicht & Adipositas $1^{\circ}$ & Adipositas $\|/\| \|^{\circ}$ \\
\hline & $\mathrm{BMl}<18,5$ & $\mathrm{BMI}>18,5$ & $\mathrm{BMI}>25$ & $\mathrm{BMl}>30$ & $\mathrm{BMl}>35$ \\
\hline Anteil Mütter [\%] & 0,31 & 27,3 & 29,14 & 26,07 & 17,18 \\
\hline Anteil Väter [\%] & 0 & 21,57 & 39,87 & 25,16 & 13,4 \\
\hline
\end{tabular}

Tabelle 16: Gewichtsverteilung der Eltern

Ein signifikant höherer durchschnittlicher BMI-SDS als für die Kinder normalgewichtiger Mütter und Väter zeigte sich für die Gruppen der Kinder und Jugendlichen, deren Mütter einen BMI über $30 \mathrm{~kg} / \mathrm{m}^{2}$ oder deren Väter einen BMI über $35 \mathrm{~kg} / \mathrm{m}^{2}$ haben.

Der mittlere mütterliche BMI mit 29,18 $\pm 6,30 \mathrm{~kg} / \mathrm{m}^{2}$ und der Mittelwert der Väter mit 29,29 \pm $5,36 \mathrm{~kg} / \mathrm{m}^{2}$ liegen etwa im selben, gerade noch übergewichtigen Bereich.

Bei der Darstellung im Boxplot (Abb. 20) kann man den Einfluss des mütterlichen Gewichts auf den BMI-SDS der Kinder und seine mit dem BMI zunehmende Bedeutung gut erkennen. Der BMI der Väter bleibt länger ohne Einfluss auf den kindlichen BMI-SDS. In der Gruppe Väter mit BMI über $35 \mathrm{~kg} / \mathrm{m}^{2}$ (Adipositas $\|^{\circ}$ oder II I9) lässt sich dann allerdings ein deutlicher Sprung des kindlichen BMI-SDS nach oben erkennen. 


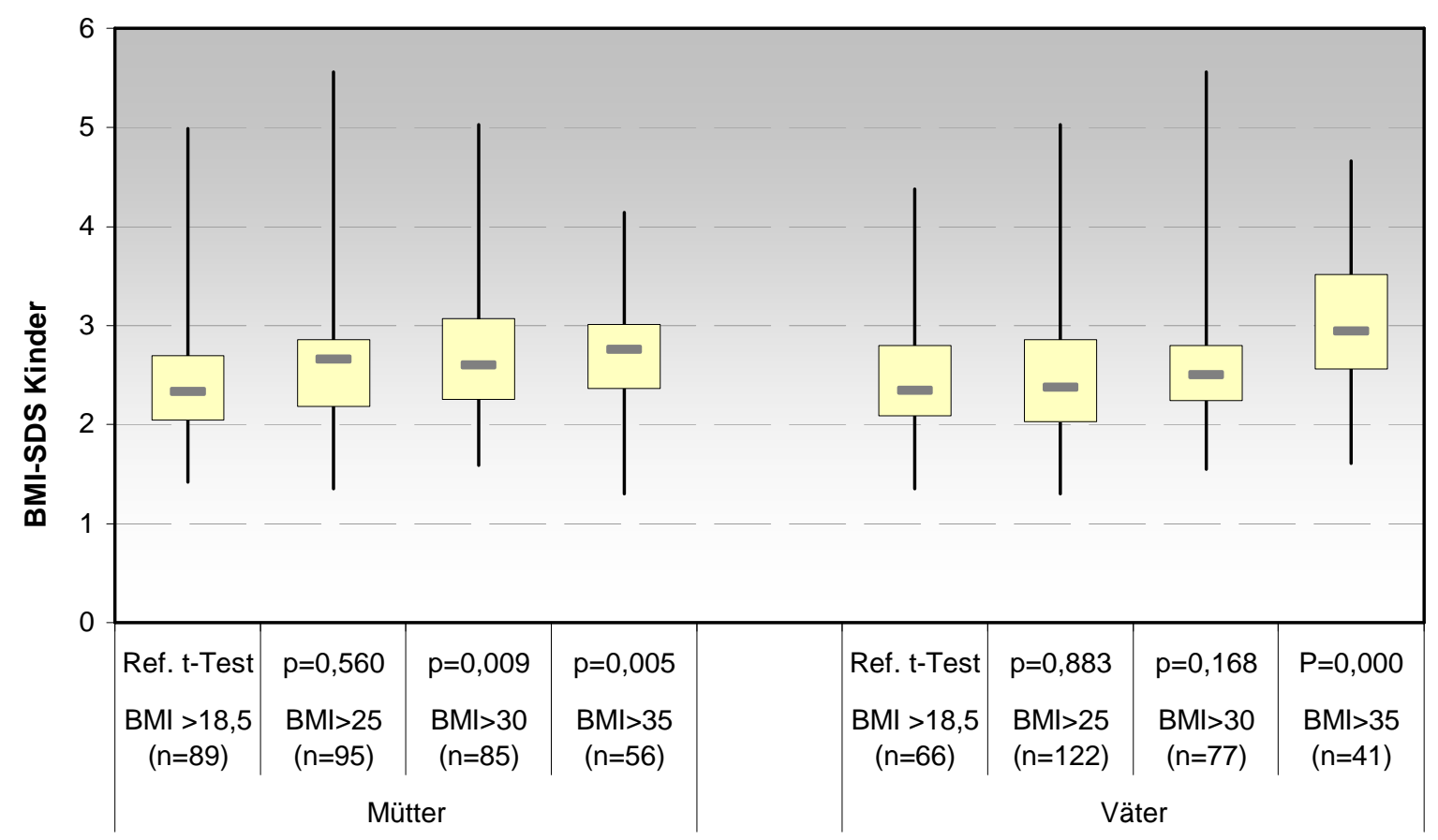

Abbildung 20: BMI-SDS bei Erstvorstellung nach BMI der Eltern

Betrachtet man beide Elternteile gemeinsam, so fällt auf, dass weniger als $10 \%$ der adipösen Kinder und Jugendlichen eine normgewichtige Mutter und einen normgewichtigen Vater haben. Bei 19,03\% ist nur die Mutter übergewichtig oder adipös und 59,86\% der Patienten haben zwei Elternteile mit einen BMI über $25 \mathrm{~kg} / \mathrm{m}^{2}$. Diese Kinder und Jugendlichen sind auch mit $\mathrm{p}=0,03$ signifikant dicker, als die, deren Eltern normgewichtig sind.

\begin{tabular}{|c|c|c|c|c|}
\hline & $\begin{array}{l}\text { BMl beider } \\
\text { Eltern }<25\end{array}$ & $\begin{array}{l}\text { BMl des } \\
\text { Vaters } \geq 25\end{array}$ & $\begin{array}{l}\text { BMl der } \\
\text { Mutter } \geq 25\end{array}$ & $\begin{array}{l}\text { BMI beider } \\
\text { Eltern } \geq 25\end{array}$ \\
\hline n Kinder & 27 & 34 & 55 & 173 \\
\hline$\%$ & 9,34 & 11,76 & 19,03 & 59,86 \\
\hline$\varnothing \mathrm{BMI}-\mathrm{SDS} \mathrm{K}$ & $2,36 \pm 0,60$ & $0,62 \pm 0,69$ & $2,45 \pm 0,64$ & $2,66 \pm 0,66$ \\
\hline$p$ & Ref. & 0,12 & 0,51 & 0,03 \\
\hline
\end{tabular}

Tabelle 17: Gemeinsame Betrachtung des BMI beider Elternteile

Die Ergebnisse der linearen Korrelationsanalyse des elterlichen BMI-SDS mit dem der Patienten ist in Tabelle 18 dargestellt. Die höchste Korrelation mit $r=0,355$ und $p=0,000$ fand sich bei Müttern mit ihren Söhnen.

\begin{tabular}{|c|c|c|c|c|c|c|}
\hline \multicolumn{7}{|c|}{ lineare Regression } \\
\hline & Mütter & & & Väter & & \\
\hline & alle Kinder & Töchter & Söhne & alle Kinder & Töchter & Söhne \\
\hline$r$ & 0,234 & 0,144 & 0,355 & 0,228 & 0,254 & 0,172 \\
\hline $\mathrm{p}$ & 0,000 & 0,072 & 0,000 & 0,000 & 0,002 & 0,029 \\
\hline
\end{tabular}

Tabelle 18: Korrelation des BMI-SDS von Eltern und ihren Kindern in der linearen Regression des BMI-SDS 


\subsubsection{Ausbildung und Berufstätigkeit der Eltern}

Der mit 45,02\% größte Teil der Mütter verließ die Schule mit einem Realschul-, der größte Teil der Väter $(43,28 \%)$ mit einem Hauptschulabschluss.

Bei Einteilung der Kinder in Gruppen nach der Schulbildung ihrer Eltern lässt sich erkennen, dass der Median des BMI-SDS mit steigendem Bildungsstand bei Müttern und Vätern abnimmt. Der Unterschied zwischen den Gruppen ist allerdings nicht signifikant (Abb. 21).

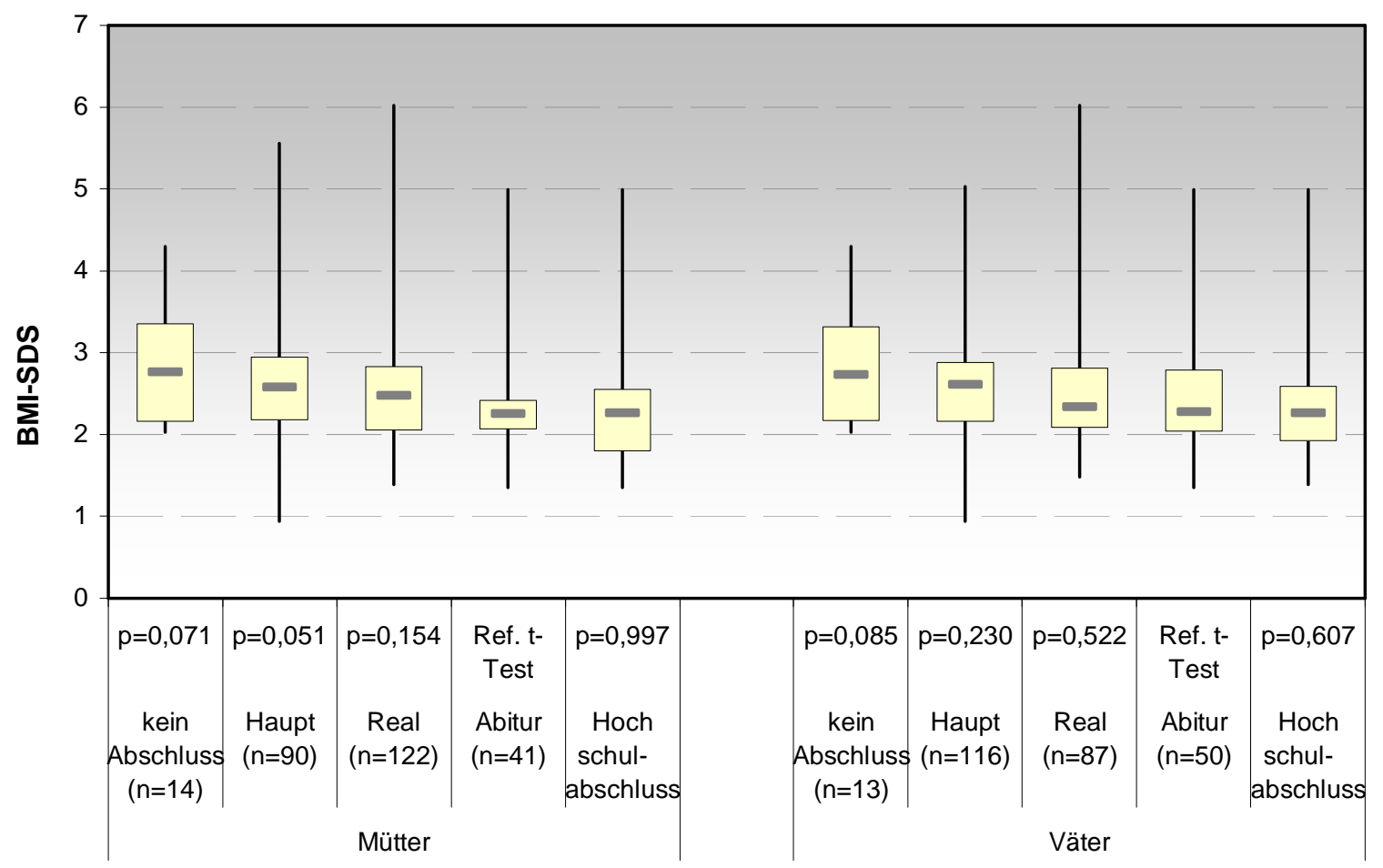

Abbildung 21: BMI-SDS bei Erstvorstellung nach Schulbildung der Eltern

Bildet man zwei Gruppen der Kinder von Müttern mit und ohne Abitur, so haben die Abiturientinnen Kinder mit einem signifikant niedrigeren BMI-SDS ( $p=0,0467)$. Für Väter mit Abitur konnte kein signifikanter Unterschied nachgewiesen werden.

Anders bei der Berufstätigkeit: bei der Betrachtung von 296 Müttern und 242 Vätern waren $56,76 \%$ der Mütter und 76,03\% der Väter voll oder halbtags beschäftigt. Ein Unterschied im medianen BMI-SDS der Kinder und Jugendlichen, deren Mütter oder Väter berufstätig sind, zu denen, deren Eltern zu Hause sind, konnte dabei nicht gezeigt werden. Der Mittelwert für den BMI-SDS ihrer Kinder lag bei berufstätigen Müttern bei 2,56 $\pm 0,64$ und bei Kindern von Hausfrauen bei 2,62 $\pm 0,74$ ( $p=0,47)$. Die Gruppe der Patienten mit Vollzeit beschäftigten Vätern hatte einen mittleren BMI-SDS von 2,56 $\pm 0,72$ und die der Kinder und Jugendlichen mit arbeitslosen oder geringfügig beschäftigten Vätern von 2,60 $\pm 0,73(p=0,74)$. 


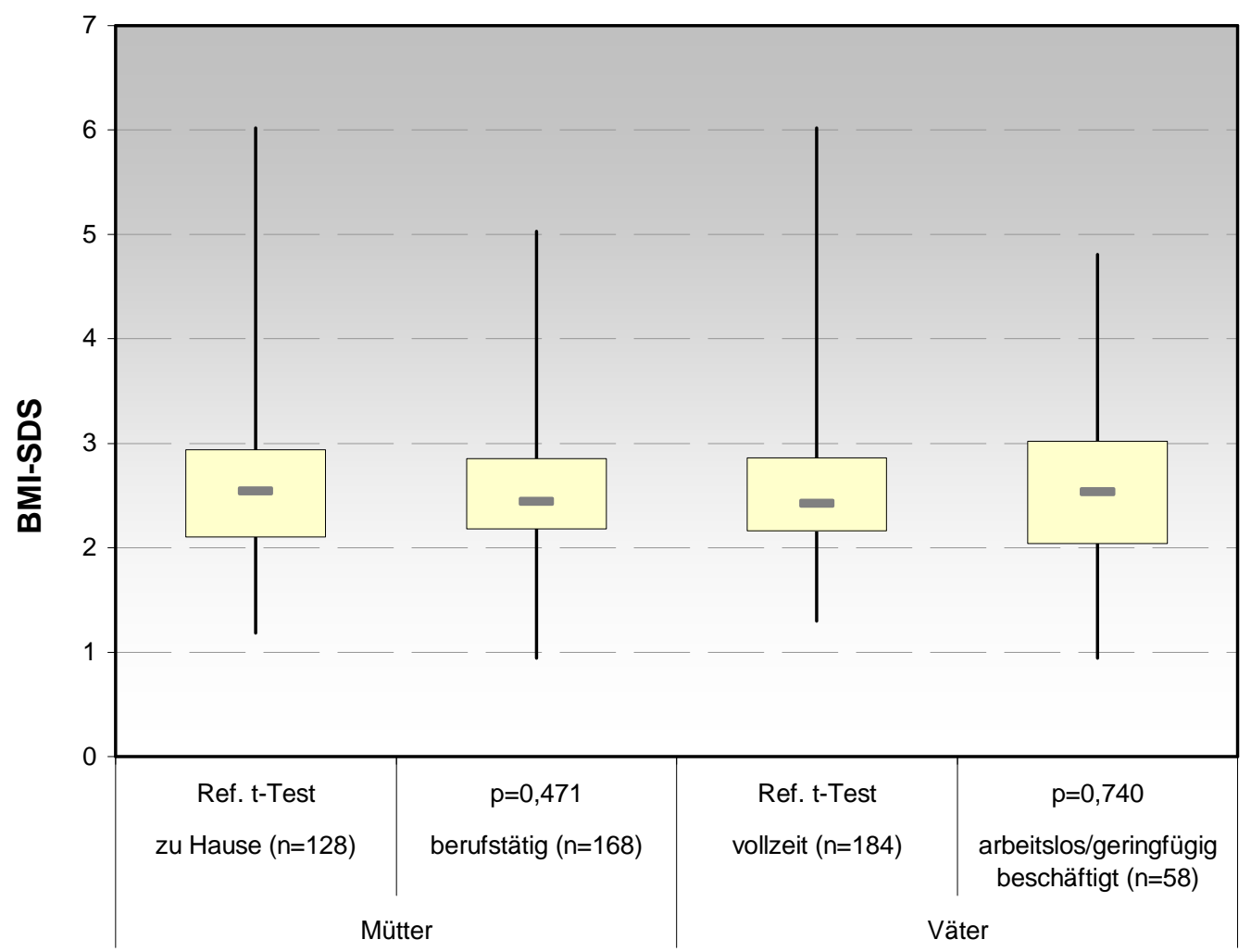

Abbildung 22: BMI-SDS bei Erstvorstellung nach Berufstätigkeit der Eltern

\subsubsection{Schulbildung der Kinder und Jugendlichen}

Die Schulform wurde bei insgesamt 376 Kindern erfasst. Bei der Erstvorstellung besuchten $50,14 \%$ eine weiterführende Schule, davon 24,46\% die Hauptschule, 38,04\% die Realschule, 21,74\% das Gymnasium und 15,76\% eine Gesamtschule. 30,25\% gingen insgesamt noch zur Grundschule, 9,26\% besuchten eine Sonderschule und 10,35\% waren noch nicht eingeschult.

Die Grundschüler hatten im Durchschnitt einen geringeren BMI-SDS $(2,42 \pm 0,64)$, entsprechen aber in Bezug auf das Alter nicht den anderen Gruppen und sind somit nicht direkt vergleichbar. Die Sonderschüler mit einem durchschnittlichen BMI-SDS 2,54 $\pm 0,62$ stellen das Alter betreffend die heterogenste Gruppe dar.

Der durchschnittliche BMI-SDS liegt zwar in der Gruppe der Gymnasiasten mit 2,43 \pm 0,68 am niedrigsten, der Unterschied zu den anderen Gruppen ist allerdings nicht signifikant.

\begin{tabular}{|l|r|r|r|r|} 
& Hauptschule & Realschule & Gymnasium & Gesamtschule \\
\hline n Kinder & 45 & 70 & 40 & 29 \\
\hline Anteil [\%] & 24,45 & 38,04 & 21,74 & 15,76 \\
\hline$\varnothing$ BMI-SDS & $2,67 \pm 0,66$ & $2,49 \pm 0,60$ & $2,43 \pm 0,68$ & $2,48 \pm 0,69$ \\
\hline p t-Test & 0,10 & 0,64 & Ref. & 0,78 \\
\hline
\end{tabular}

Tabelle 19: Schulbildung der Kinder und Jugendlichen des Kollektivs 
Betrachtet man die Hauptschüler isoliert gegenüber allen anderen Patienten, konnte bei innen ein signifikant höherer BMI-SDS im Gegensatz zu den Schülern anderer Schulformen gefunden werden $(p=0,04966)$.

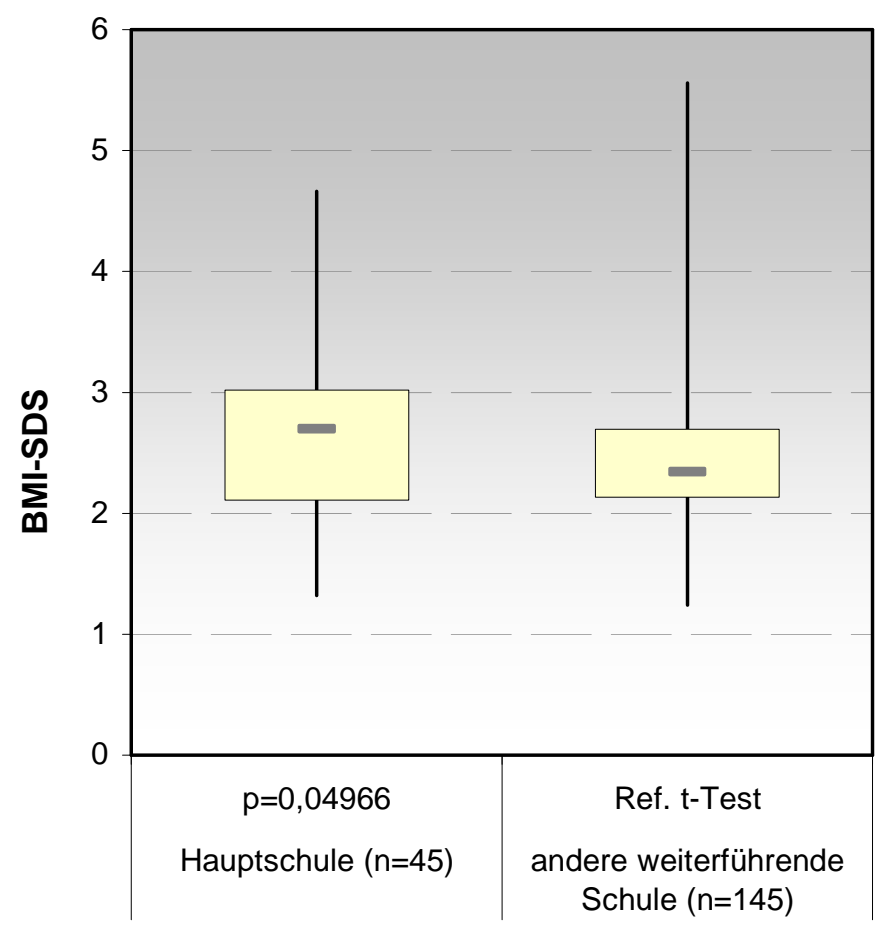

Abbildung 23: BMI-SDS nach weiterführender Schulbildung der Kinder und Jugendlichen 


\subsection{Folge- und Begleiterkrankungen}

\subsubsection{Metabolisches Syndrom}

\subsubsection{HOMA-IR als Zeichen der Insulinresistenz}

Von 136 Kindern und Jugendlichen wurde als Maß für die Insulinresistenz der HOMA-IR aus Nüchtern-Insulin und Nüchtern-Glukose berechnet. Dabei zeigte sich, dass der Wert von 52 Patienten (gesamt: 38,24\%, Mädchen: 37,31\%, Jungen: 38,03\%) oberhalb des alters- und geschlechtsspezifischen 95. Perzentils lag.

Besonders betroffen waren hiervon die älteren Mädchen von 14-18 Jahren, während bei den Jungen die Altersgruppen unter 14 Jahren dominieren.

Insgesamt lagen nur wenige HOMA-IR-Werte (18,38\%) im niedrigen Perzentilbereich unterhalb des 50. Perzentils.

\begin{tabular}{|c|c|c|c|c|c|c|}
\hline \multicolumn{7}{|l|}{ Mädchen } \\
\hline & \multirow{2}{*}{$\begin{array}{l}\text { 0-9 Jahre } \\
\text { HOMA } \\
<95 . P\end{array}$} & & \multirow{2}{*}{$\begin{array}{l}\text { 10-13 Jahre } \\
\text { HOMA } \\
<95 . P\end{array}$} & \multicolumn{3}{|c|}{ 14-18 Jahre } \\
\hline & & $\begin{array}{l}\text { HOMA } \\
>95 . P\end{array}$ & & $\begin{array}{l}\text { HOMA } \\
>95 . P\end{array}$ & $\begin{array}{l}\text { HOMA } \\
<95 . P\end{array}$ & $\begin{array}{l}\text { HOMA } \\
>95 . P\end{array}$ \\
\hline $\mathrm{n}$ & 13 & 6 & 15 & 8 & 10 & 8 \\
\hline$\%$ & 21,67 & 10,00 & 25,00 & 13,33 & 16,67 & 13,33 \\
\hline$\varnothing \mathrm{BMI}-S D S$ & $2,93 \pm 0,61$ & $2,34 \pm 0,49$ & $2,30 \pm 0,6$ & $2,68 \pm 0,66$ & $2,58 \pm 0,42$ & $3,15 \pm 0,42$ \\
\hline$p$ & Ref. & 0,055 & Ref. & 0,178 & Ref. & 0,012 \\
\hline
\end{tabular}

\begin{tabular}{|c|c|c|c|c|c|c|}
\hline \multicolumn{7}{|l|}{ Jungen } \\
\hline & $\begin{array}{l}\text { 0-9 Jahre } \\
\text { HOMA } \\
<95 . P\end{array}$ & $\begin{array}{l}\text { HOMA } \\
>95 . P\end{array}$ & $\begin{array}{l}\text { 10-13 Jahre } \\
\text { HOMA } \\
<95 . P\end{array}$ & $\begin{array}{l}\text { HOMA } \\
>95 . P\end{array}$ & $\begin{array}{l}\text { 14-18 Jahre } \\
\text { HOMA } \\
<95 . P\end{array}$ & $\begin{array}{l}\text { HOMA } \\
>95 . P\end{array}$ \\
\hline$n$ & 13 & 9 & 16 & 10 & 15 & 6 \\
\hline$\%$ & 18,84 & 13,04 & 23,19 & 14,49 & 21,74 & 8,70 \\
\hline$\varnothing$ BMI-SDS & $2,22 \pm 0,31$ & $2,97 \pm 0,56$ & $2,29 \pm 0,31$ & $2,58 \pm 0,26$ & $2,36 \pm 0,58$ & $2,94 \pm 0,47$ \\
\hline$p$ & Ref. & 0,001 & Ref. & 0,020 & Ref. & 0,042 \\
\hline
\end{tabular}

Tabelle 20: Insulinresistenz in \% nach Alter und Geschlecht

Der Zusammenhang eines pathologischen HOMA-IR Wertes oberhalb des 95. Perzentils und dem BMI-SDS wurde für 129 Kinder und Jugendliche ermittelt.

Bei den meisten Gruppen zeigte sich erwartungsgemäß ein höherer mittlerer BMI-SDS für Patienten mit erhöhtem HOMA-IR. Dieser Unterschied ist bei den Jungen in allen Altersgruppen, bei den Mädchen nur im Alter von 14-18 Jahren signifikant.

Die Mädchen zwischen null und neun Jahren oberhalb des 95. Perzentils für den HOMA-IR haben entgegen den Erwartungen einen niedrigeren medianen BMI-SDS als die Mädchen mit unauffälligen Werten. 


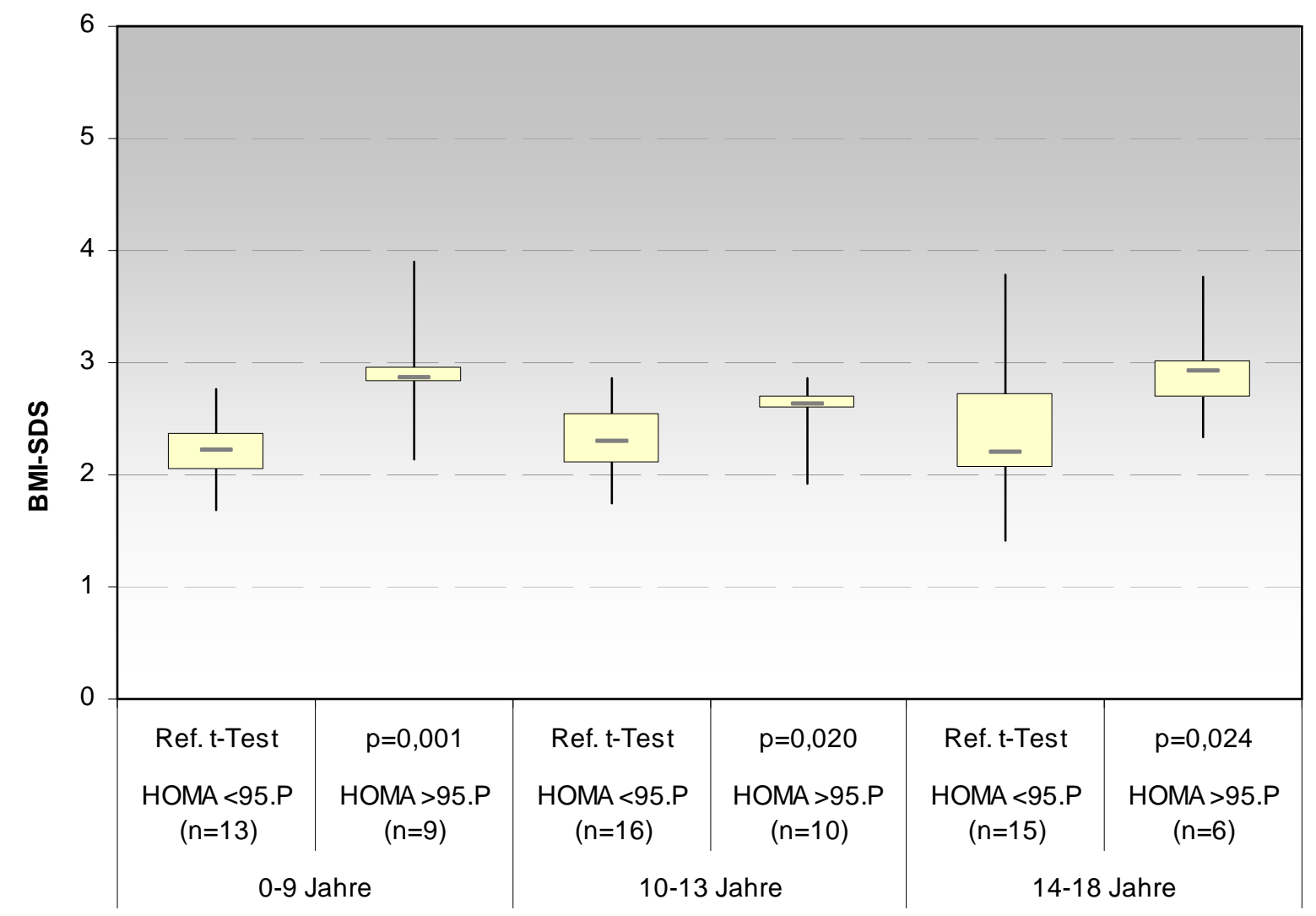

Abbildung 24: HOMA-Werte und BMI-SDS der Jungen

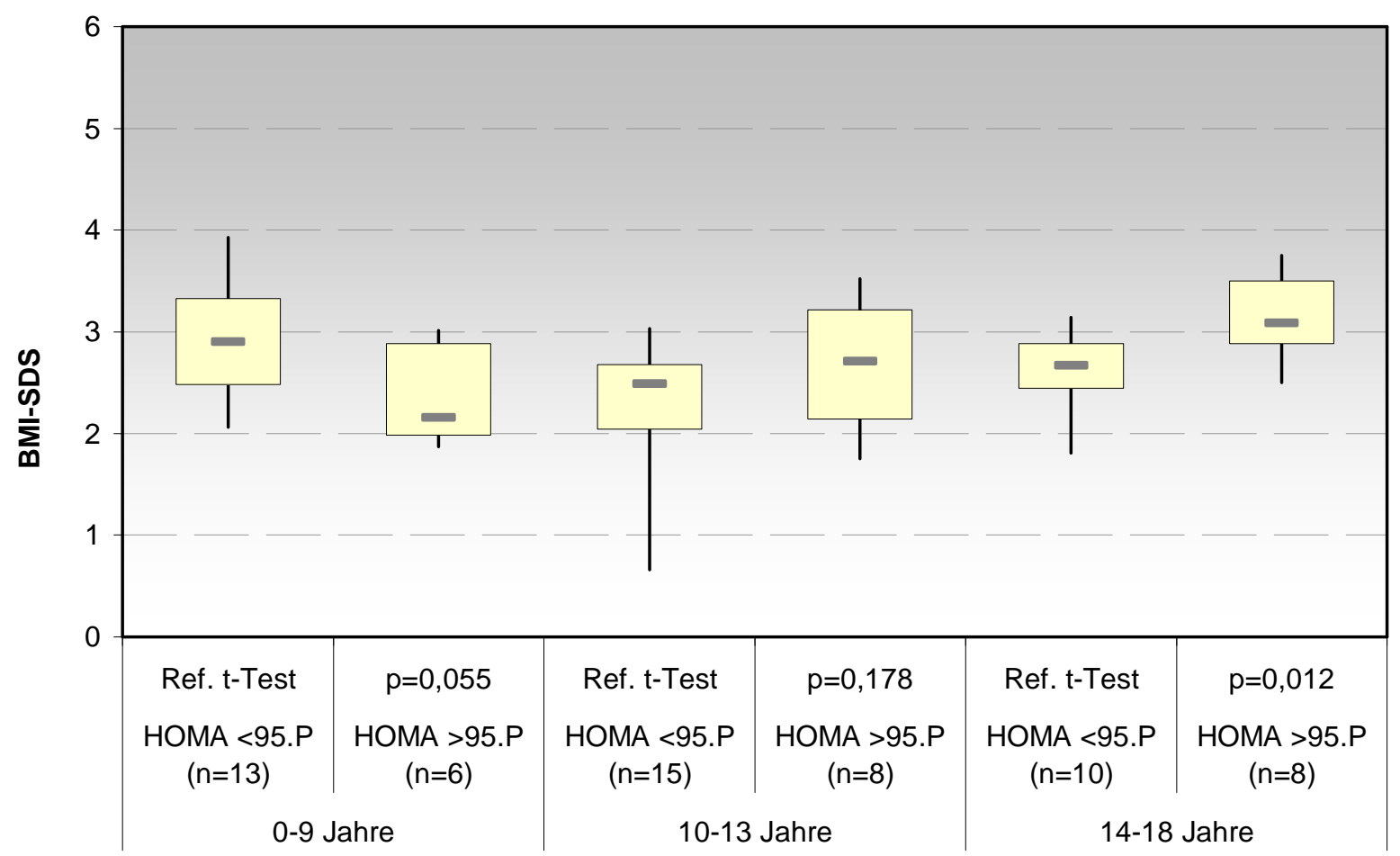

Abbildung 25: HOMA-Werte und BMI-SDS der Mädchen 


\subsubsection{Störungen der Glukosetoleranz}

Zum Screening auf Störungen der Glukosetoleranz wurde bei allen Patienten standardmäßig die Nüchtern-Glukose (Glukose $\mathrm{t}=0$ ) bestimmt. Hier liegen die Werte von der Erstvorstellung von 376 Kindern und Jugendlichen vor. Dabei waren insgesamt 19 Werte (5,05\%) auffällig, 13 bei den Jungen und 6 bei den Mädchen, was einen geschlechtsbezogenen prozentualen Anteil von 6,57 bei Jungen, bzw. 3,37 bei den Mädchen ergibt. Die Nüchternglukose war bei

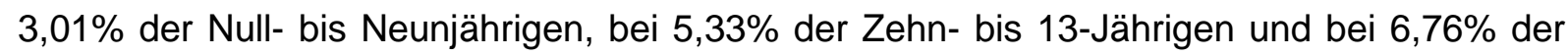
14-18-Jährigen getesteten Kinder erhöht.

Bei gegebenem klinischem Verdacht wurde zusätzlich ein oraler Glukosetoleranztest (OGTT) durchgeführt. Der Glukose-Wert nach 2 Stunden (Glukose $t=120$ ) liegt von 52 Mädchen und 39 Jungen vor. Bei einem Großteil der Kinder (Mädchen: 88,54\%, Jungen: 76,92\%) waren sowohl der Nüchtern- als auch der 2-Stunden-Glukosewert unauffällig. 13,46\% der Mädchen und sogar 23,08\% der Jungen hatten mindestens einen auffälligen Glukosewert im Sinne einer gestörten Glukosetoleranz.

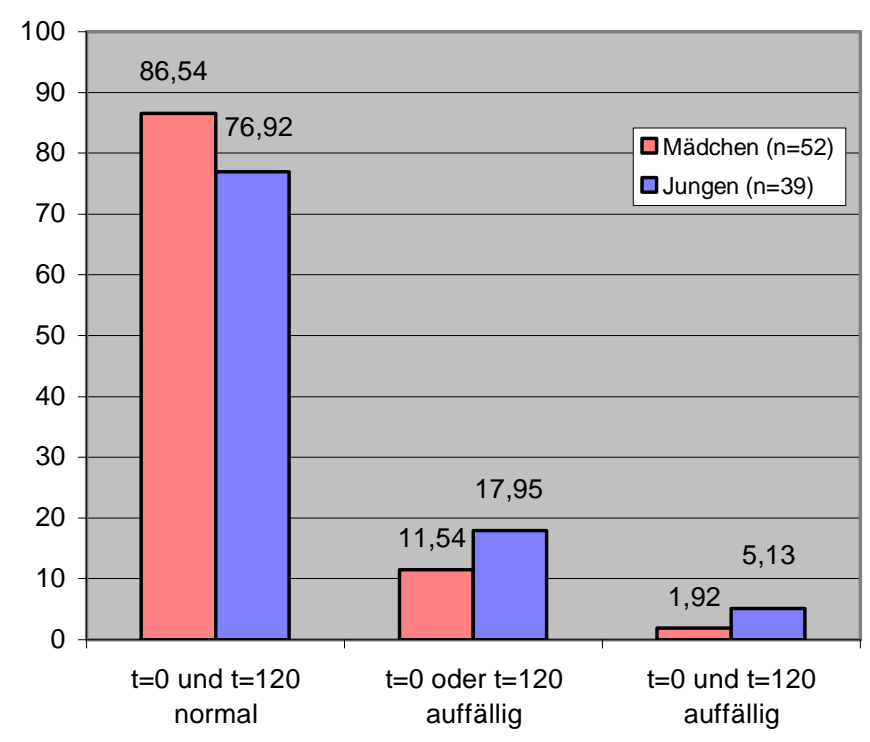

Abbildung 26: Anteil auffälliger Werte im OGTT bei Nüchtern-Glukose und 2-Stunden-Wert

Bezogen auf alle 376 Kinder und Jugendliche, deren Nüchternglukose gescreent wurde, hatten 3,37\% der Mädchen und 3,03\% der Jungen, davon kein Kind in der Altersgruppe 0-9 Jahre, 4,73\% der 10-13-Jährigen und 5,41\% der 14-18-Jährigen einen auffälligen OGTT im Sinne einer gestörten Glukosetoleranz.

Ein manifester Diabetes mellitus mit einer Nüchtern-Glukose über $126 \mathrm{mg} / \mathrm{dl}$ und einem Zwei-Stunden-Wert über 200 mg/dl fanden wir nur in einem Fall. Betroffen war ein 14jähriger Junge mit einem BMI-SDS von 2,93. 


\subsubsection{Steatosis hepatis}

Die Transaminasen (AST, ALT) wurden bei der Erstvorstellung bei 217 Jungen und 188 Mädchen ermittelt. Der größte Teil der Werte lieferte keinen Hinweis auf eine Fettlebererkrankung, mit dem Alter nahm jedoch bei Jungen und Mädchen der Anteil auffälliger Werte zu (AST oder ALT > $50 \mathrm{U} / \mathrm{l}$ ). Insgesamt hatten 10,57\% (Jungen: 15,21\%, Mädchen: 4,26\%) der Kinder mindestens einen auffälligen Wert. Die Jungen waren stärker betroffen als die Mädchen, besonders in den Altersgruppen ab zehn Jahren. In der Gruppe der 14-18-jährigen Jungen zeigten sich bei $22,86 \%$ erhöhte Serumtransaminasen.

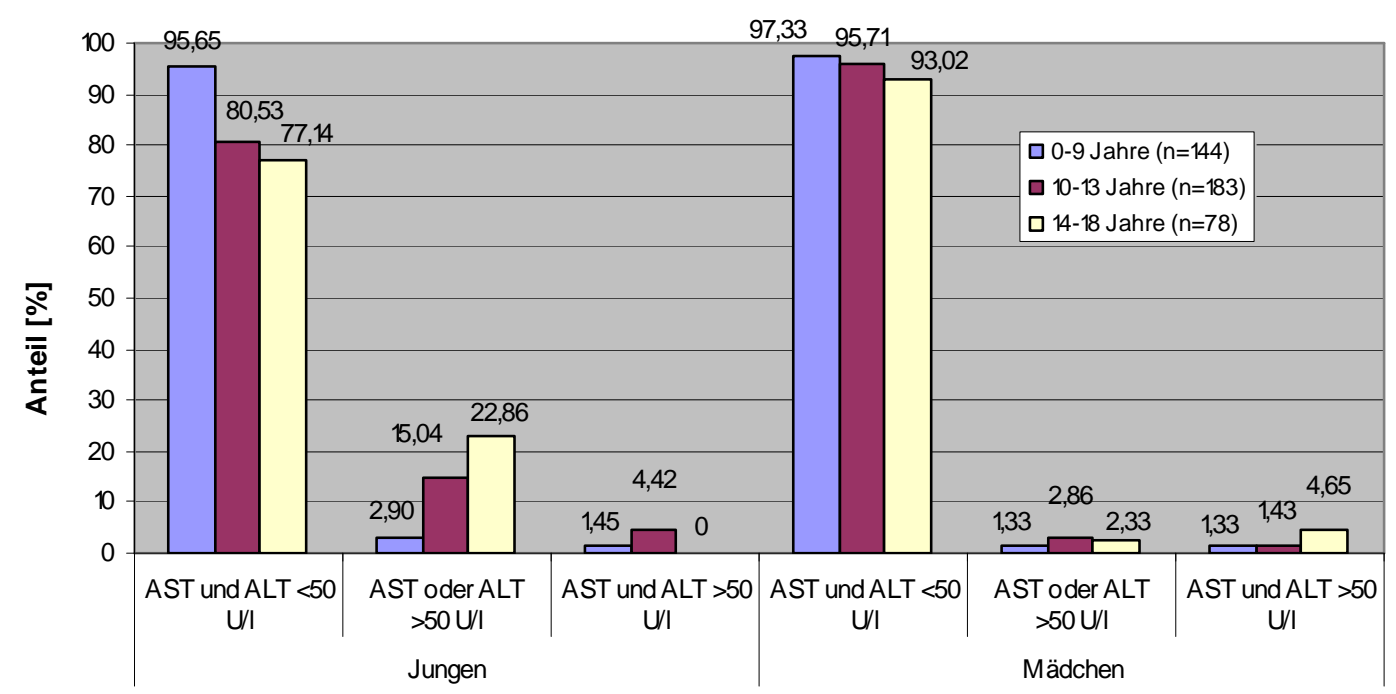

Abbildung 27: Transaminasen bei Erstvorstellung

Betrachtet man den BMI-SDS und das Alter der betroffenen Patienten, so sind diese durchschnittlich älter und haben einen höheren BMI-SDS als die gesunden Kinder und Jugendlichen. Signifikant war allerdings nur die Abhängigkeit vom Alter bei den Jungen mit $p=0,006$.

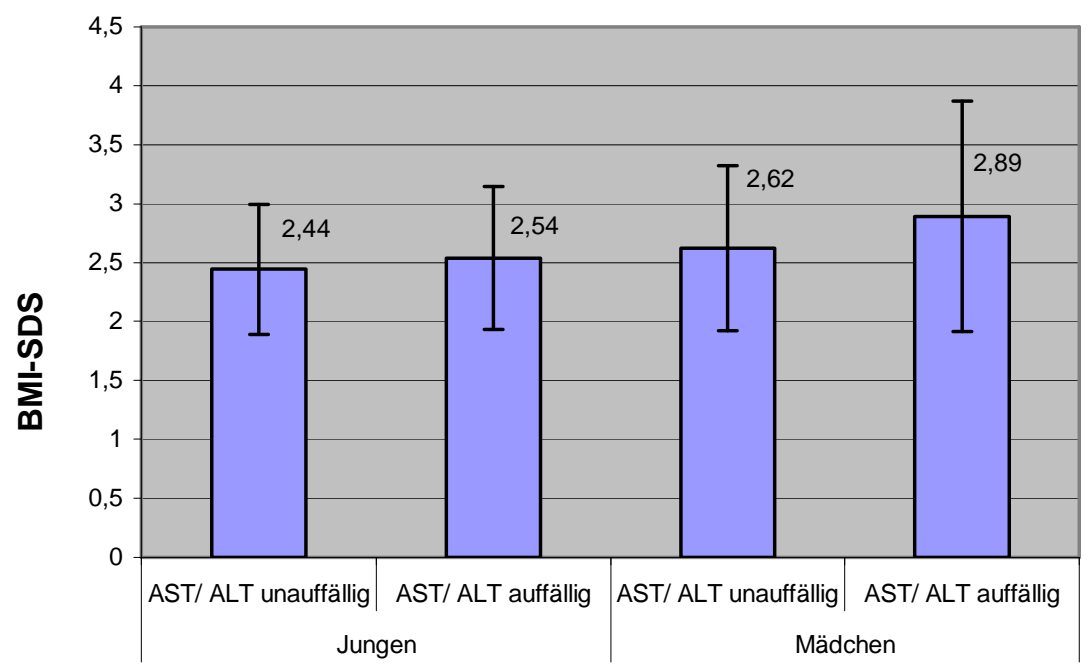

Abbildung 28: Transaminasen und durchschnittlicher BMI-SDS bei Erstvorstellung 


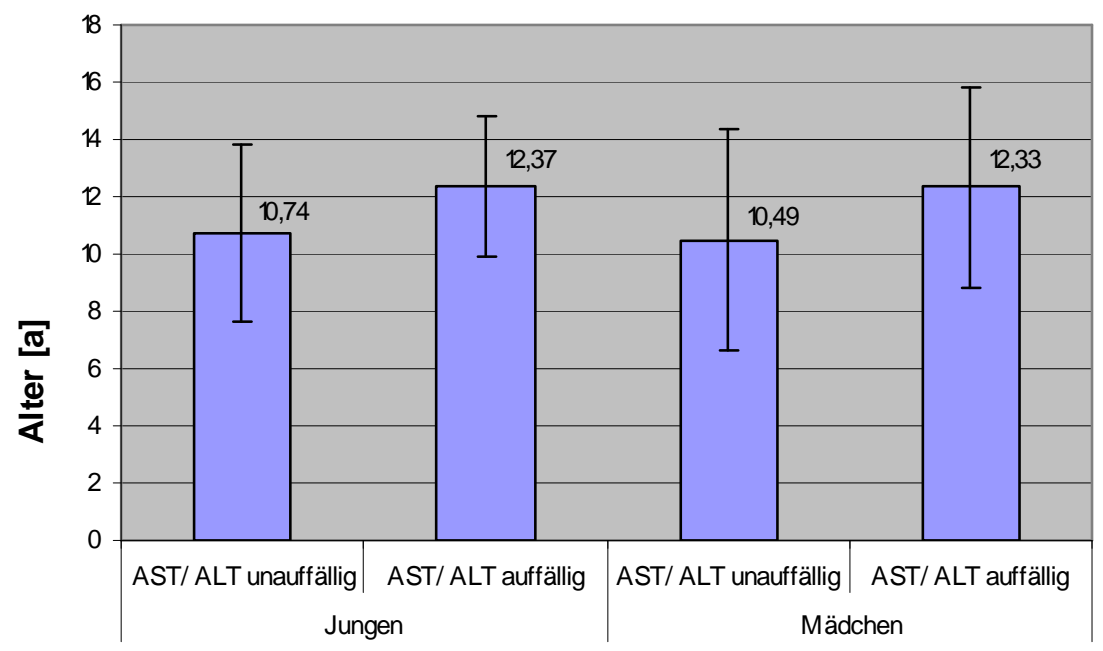

Abbildung 29: Transaminasen und durchschnittliches Alter bei Erstvorstellung

\subsubsection{Fettstoffwechsel}

Bei der Auswertung der Lipoproteinwerte LDL, Triglyceride (TG) und Gesamtcholesterin der Kinder und Jugendlichen des Kollektivs zeigte sich ein hoher Anteil an Werten oberhalb des alters- spezifischen Normwertes.

\subsection{Triglyceride}

\begin{tabular}{|c|c|c|c|c|c|c|}
\hline \multicolumn{7}{|l|}{ Mädchen } \\
\hline & 0-9 Jahre & & 10-13 Jahre & & 14-18 Jahre & \\
\hline & TG normal & TG erhöht & TG normal & TG erhöht & TG normal & TG erhöht \\
\hline $\mathrm{n}$ & 40 & 28 & 27 & 38 & 21 & 15 \\
\hline$\%$ & 58,82 & 41,18 & 41,54 & 58,46 & 58,33 & 41,67 \\
\hline$\varnothing$ BMI-SDS & $2,63 \pm 0,58$ & $2,36 \pm 0,63$ & $2,63 \pm 0,83$ & $2,46 \pm 0,62$ & $2,90 \pm 0,76$ & $2,67 \pm 0,56$ \\
\hline p t-Test & Ref. & 0,075 & Ref. & 0,343 & Ref. & 0,327 \\
\hline
\end{tabular}

\begin{tabular}{|c|c|c|c|c|c|c|}
\hline \multicolumn{7}{|l|}{\begin{tabular}{|l} 
Jungen \\
\end{tabular}} \\
\hline & 0-9 Jahre & & 10-13 Jahre & & 14-18 Jahre & \\
\hline & TG normal & TG erhöht & TG normal & TG erhöht & TG normal & TG erhöht \\
\hline$n$ & 37 & 20 & 51 & 49 & 17 & 15 \\
\hline$\%$ & 64,91 & 35,09 & 51,00 & 49,00 & 53,13 & 46,88 \\
\hline$\varnothing$ BMI-SDS & $2,57 \pm 0,66$ & $2,92 \pm 0,66$ & $2,35 \pm 0,49$ & $2,34 \pm 0,43$ & $2,62 \pm 0,62$ & $2,35 \pm 0,45$ \\
\hline pt-Test & Ref. & 0,065 & Ref. & 0,983 & Ref. & 0,173 \\
\hline
\end{tabular}

Tabelle 21: Triglyceride bei Erstvorstellung nach Alter und Geschlecht

Die Triglyceride wurden von 169 Mädchen und 189 Jungen ermittelt. Insgesamt waren 46,09\% der Kinder von erhöhten TG-Werten betroffen (Mädchen 47,93\%, Jungen 44,44\%). 
Hier lässt sich jedoch für unser Kollektiv kein Zusammenhang mit der Höhe des BMI-SDS oder dem Alter nachweisen. Erhöhte Serumtriglyceride fanden sich je nach Altersgruppe und Geschlecht bei zwischen 35,09\% bei den Jungen unter 10 Jahren und 58,46\% bei den Mädchen zwischen zehn und 13 Jahren.

\subsection{Lipoproteine}

Die Werte für LDL- und Gesamtcholesterin befinden sich zwar oberhalb des Normwertes, zum größten Teil (zwischen 50,00\% und 92,31\%) jedoch lediglich in einem moderat erhöhten Bereich bis zu $120 \%$ des oberen Normwertes. Das HDL ist zu einem relevanten und mit dem Alter steigenden Anteil erniedrigt. Bei den 14-18-jährigen Jugendlichen sind beide Geschlechter etwa zu einem Drittel betroffen. Ein signifikanter Zusammenhang des HDLCholesterin-Spiegels mit dem BMI-SDS konnte hier nicht nachgewiesen werden.

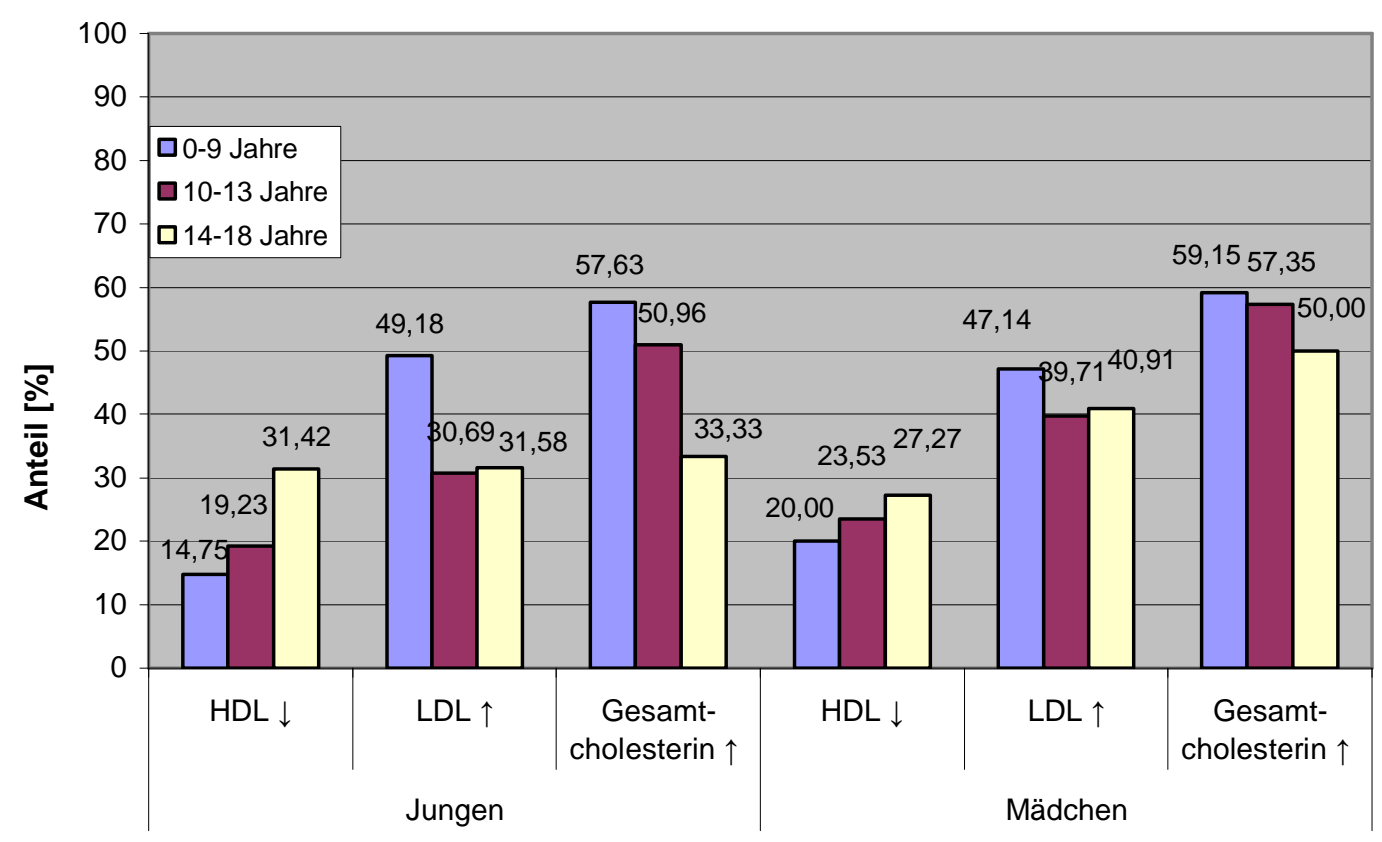

Abbildung 30: Cholesterin bei Erstvorstellung nach Alter und Geschlecht

\subsubsection{Arterielle Hypertonie}

Der Blutdruck wurde bei jeder Vorstellung standardmäßig gemessen. Von der Erstvorstellung liegen die Werte von 203 Jungen und 219 Mädchen vor. Bei 16,26\% der Jungen und 9,13\% der Mädchen ergab sich daraus der Verdacht auf eine arterielle Hypertonie, zu dessen Ausschluss bei insgesamt 52 Kindern und Jugendlichen eine 24Stunden- Blutdruckmessung vorgenommen wurde. 


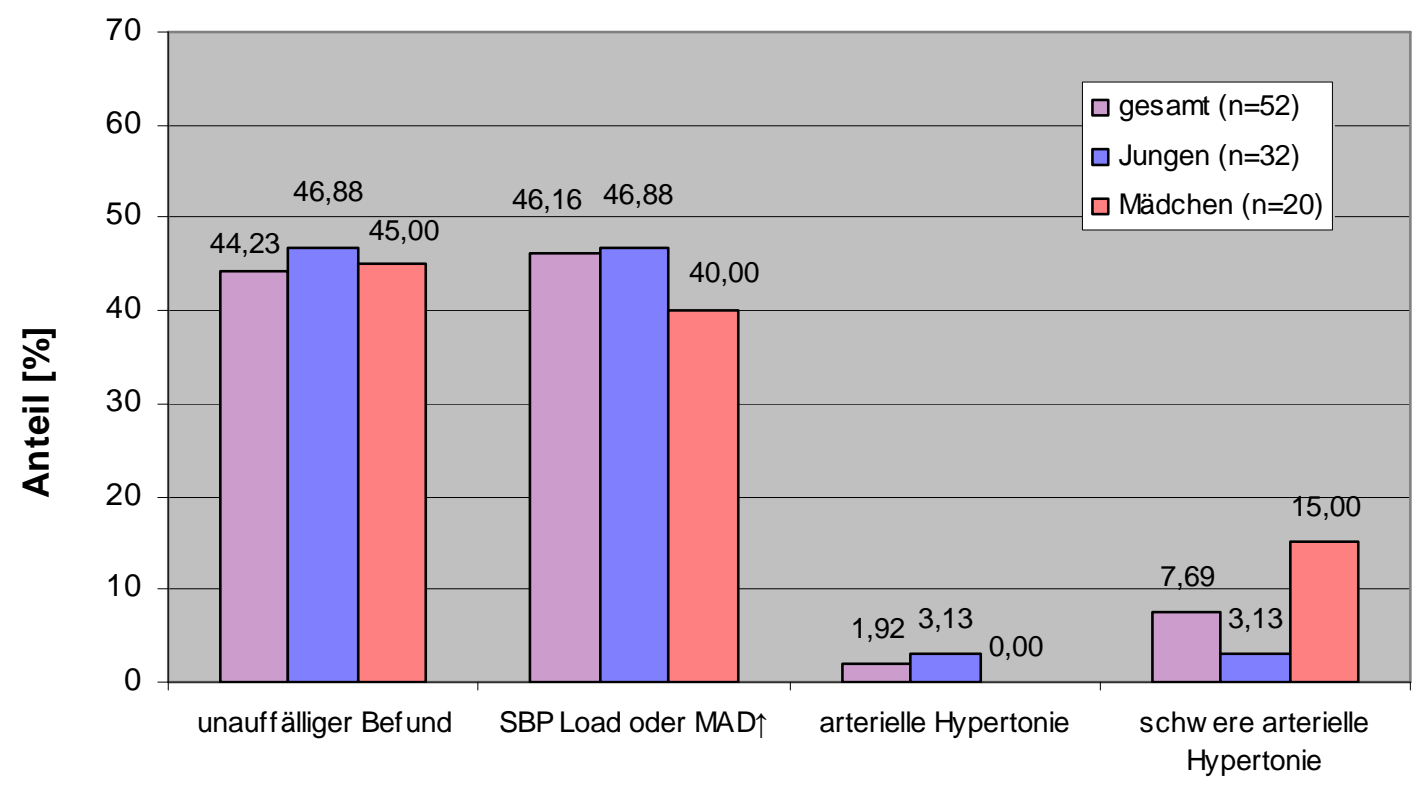

Abbildung 31: Anteil pathologischer 24-Stunden-Blutdruckmessungen

Die betroffenen Jungen hatten ein durchschnittliches Alter von 11,93 $\pm 2,98$, die Mädchen von 10,85 $\pm 3,25$ Jahren. Kinder unter acht Jahren waren eher sehr selten betroffen. Insgesamt ergaben sich bei 55,77\% der Tests auffällige Befunde. Bei 1,92\% fand sich eine arterielle Hypertonie mit einem mittleren arteriellen Druck (MAD) oberhalb des 95. Perzentils für Körpergröße und Geschlecht und einer Blood Pressure Load über 25\%, 7,69\% der Kinder und Jugendlichen wiesen sogar eine schwere arterielle Hypertonie mit einem MAD oberhalb des 95. Perzentils und einer Blood Pressure Load über 50\% auf. Bei den verbleibenden 46,16\% waren einzelne Parameter der 24-Stunden-Blutdruckmessung erhöht, sie erfüllten jedoch nicht die Definition für eine arterielle oder sogar schwere arterielle Hypertonie. Insgesamt erfüllten drei Mädchen und zwei Jungen die Kriterien für eine arterielle Hypertonie oder schwere arterielle Hypertonie.

\subsubsection{6. Übersicht der Komponenten des metabolischen Syndroms}

In die Auswertung gingen die Komponenten des metabolischen Syndroms nach Janner et al. von 2006 (Adipositas, Insulinresistenz, vermindertes HDL, erhöhte Triglyceride, arterielle Hypertonie) und zusätzlich die von der AGA vorgeschlagenen Parameter Transaminasen und Harnsäure ein. Es konnte gezeigt werden, dass die Adipositas bereits Folgen hat: bei $60,73 \%$ der Mädchen und 63,22\% der Jungen stellten wir mindestens eine der oben genannten Folgeerkrankungen fest. Bei keinem der Patienten waren alle Parameter pathologisch. 


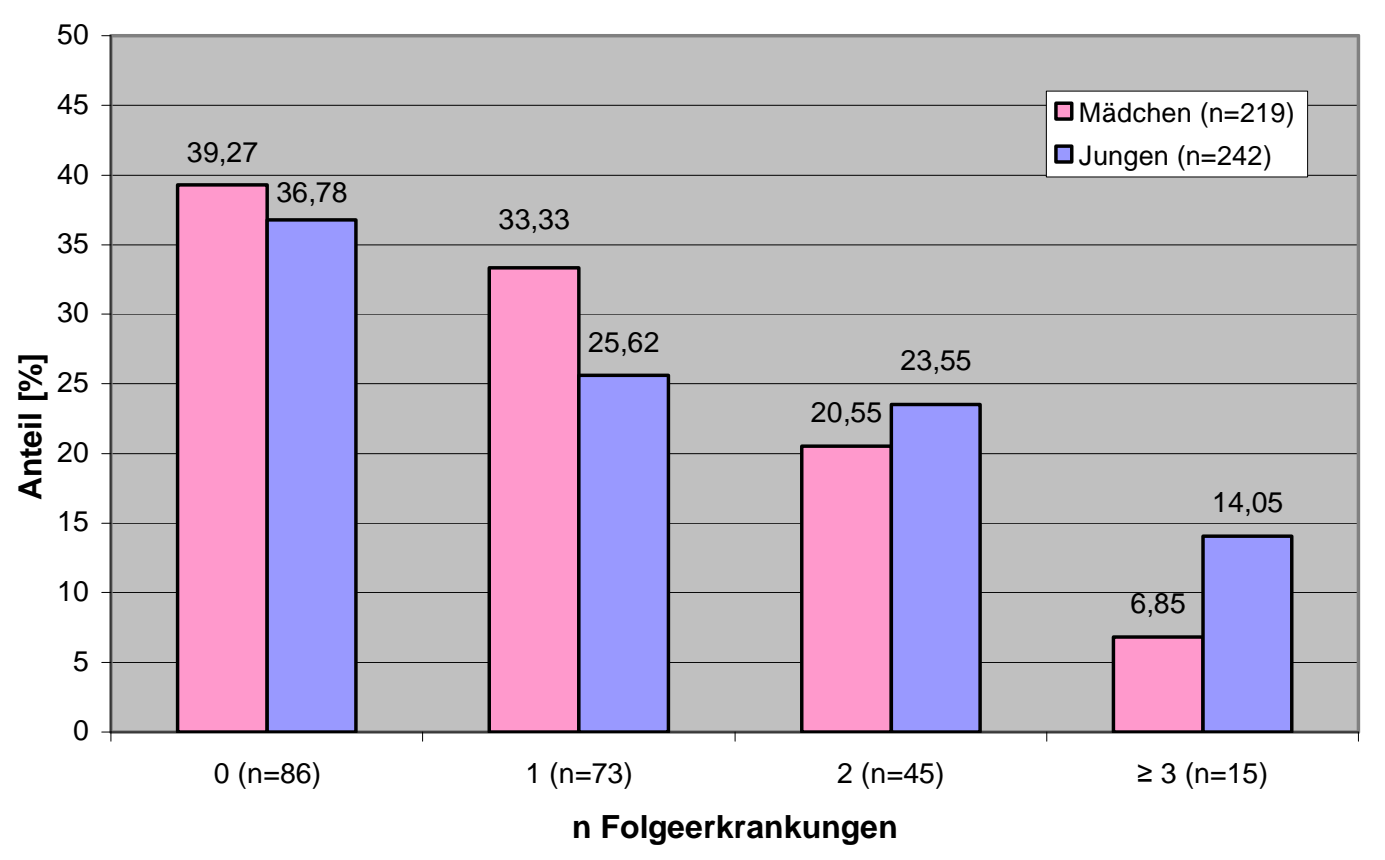

Abbildung 32: Prozentualer Anteil nach Anzahl der manifesten Folgeerkrankungen

Die Kinder wurden in Gruppen nach Anzahl der bestehenden Folgeerkrankungen eingeteilt und BMI-SDS und Alter verglichen.

Das mittlere Alter in den Gruppen, die nach Anzahl der Folgeerkrankungen eingeteilt waren, nahm mit steigender Belastung durch Folgeerkrankungen bei Jungen und Mädchen immer weiter zu. Kinder ohne Co-Morbidität waren in fast allen Gruppen signifikant jünger als Kinder, die schon an Folgeerkrankungen leiden. Die einzige Ausnahme bilden die Jungen mit nur einer Co-Morbidität. Hier ist der Altersunterschied nicht signifikant (Abb. 33).

Die Jungen ohne Co-Morbidität hatten einen signifikant niedrigeren BMI-SDS als die, die schon an Folgeerkrankungen leiden. Bei den Mädchen zeigte sich in den verschiedenen Gruppen jeweils ein ähnlicher medianer BMI-SDS (Abb. 34).

Die Definition für das metabolische Syndrom nach Janner et al. erfüllen 15,07\% der Mädchen und 15,70\% der Jungen. Diese Patienten haben mit 2,75 $\pm 0,54$ einen höheren mittleren BMI-SDS und sind mit 11,50 $\pm 3,17$ Jahren älter als die Kinder und Jugendlichen, auf die die Definition nicht zutrifft (SDS: 2,51 $\pm 0,69$, Alter: 10,69 $\pm 3,60$ ). Dieser Unterschied ist jedoch nur für den BMI-SDS bei den Jungen mit $p=0,006$ signifikant. 


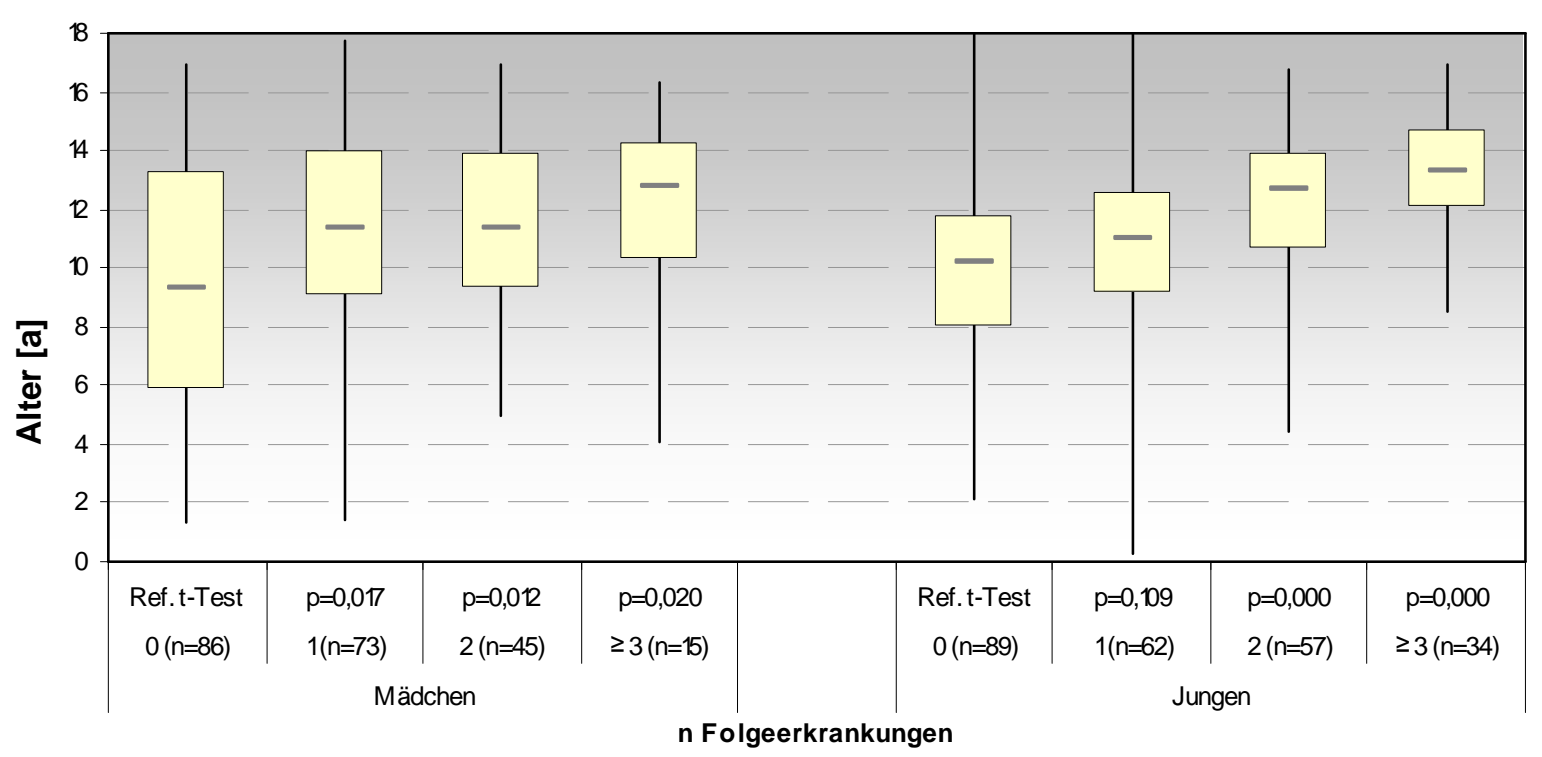

Abbildung 33: Anzahl der Folgeerkrankungen und Alter bei Jungen und Mädchen

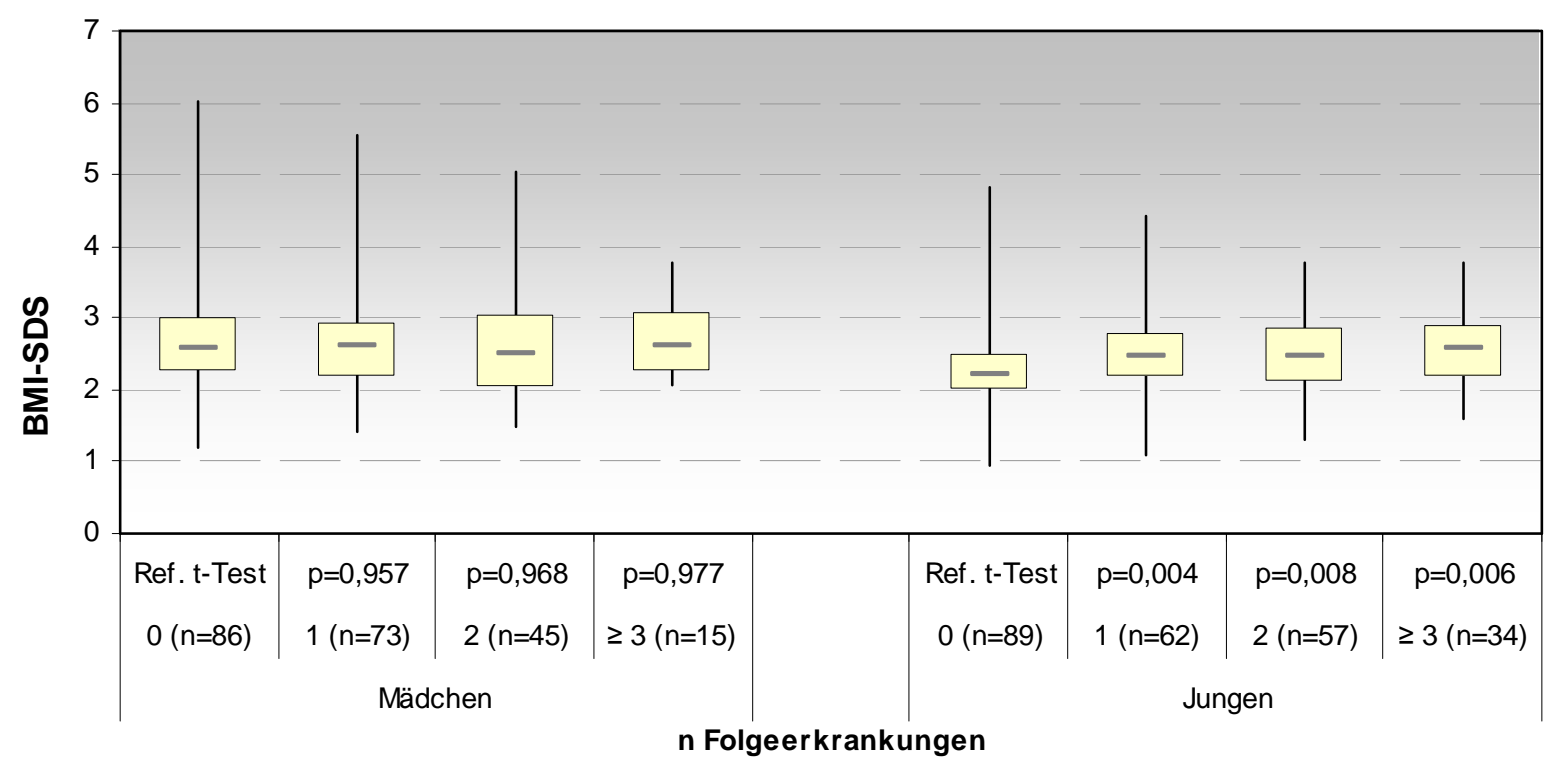

Abbildung 34: Anzahl der Folgeerkrankungen und BMI-SDS bei Jungen und Mädchen

\subsubsection{Hyperurikämie}

Der Harnsäurespiegel bei Erstvorstellung liegt von 179 Mädchen und 210 Jungen vor, wovon sich bei insgesamt 25,70\% der Mädchen und 39,05\% der Jungen erhöhte Werte zeigten.

Besonders betroffen waren die Jungen über 13 Jahren, bei denen 81,08 \% der Werte über dem oberen Referenzwert von $5,9 \mathrm{mg} / \mathrm{dl}$ lagen. Die Erhöhung der Werte in diesem Alter ist zudem gegenüber den anderen Altersgruppen signifikant höher (Abb.35).

Auch bei den Mädchen nimmt der mittlere Harnsäurespiegel mit dem Alter signifikant zu, auch wenn die Unterschiede nicht so deutlich ausgeprägt sind wie bei den Jungen. 
Ein signifikant höherer BMI-SDS der Betroffenen konnte nur für die Altersgruppe der 10-13Jährigen nachgewiesen werden.

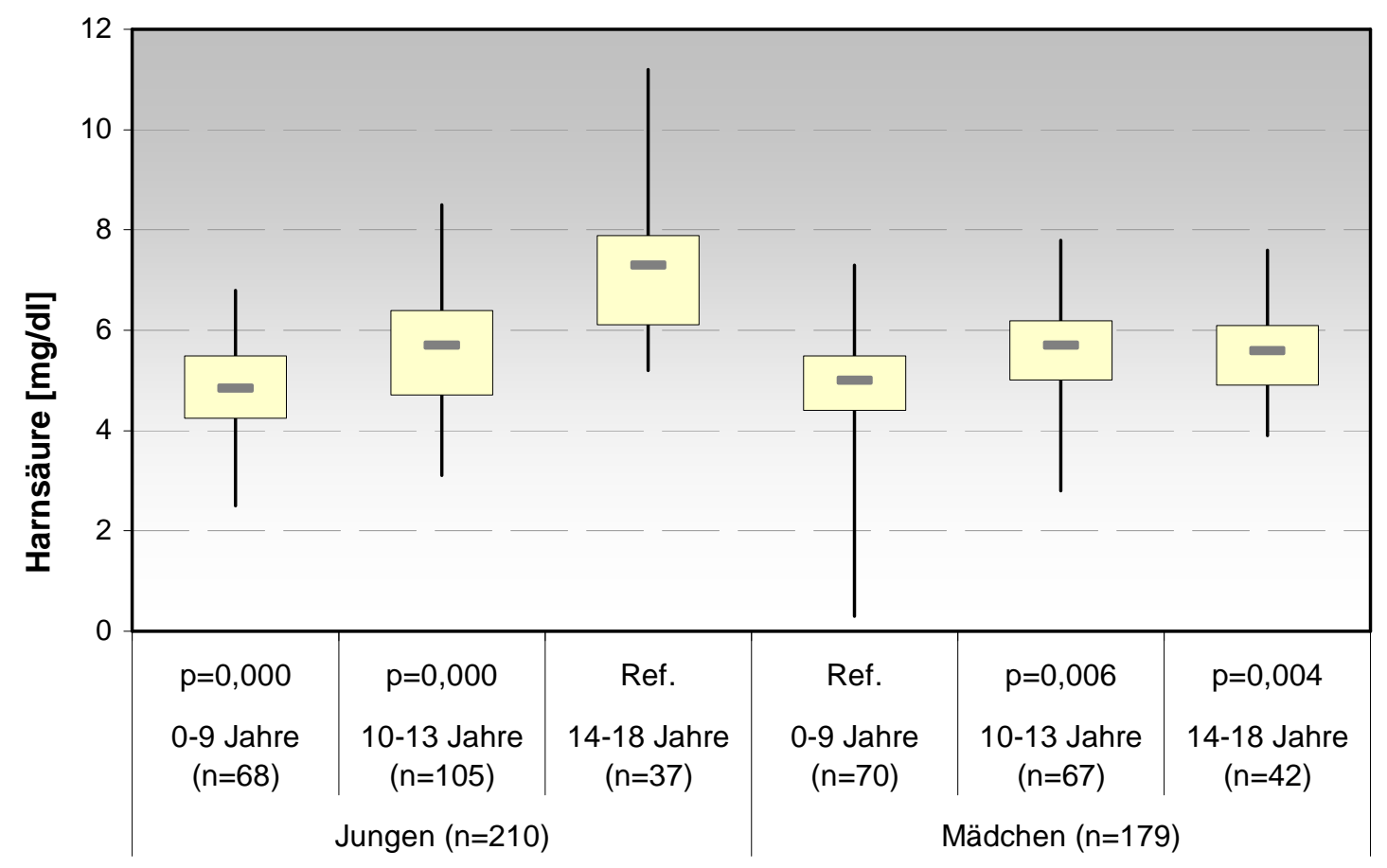

Abbildung 35: Harnsäurespiegel bei Erstvorstellung nach Alter und Geschlecht

\subsubsection{Leptin}

In der linearen Regressionsanalyse des Leptinspiegels mit dem BMI-SDS der Patienten zeigt sich bei den Jungen aller Altersgruppen eine positive Korrelation, die für die Gesamtanalyse

\section{Leptin Mädchen}

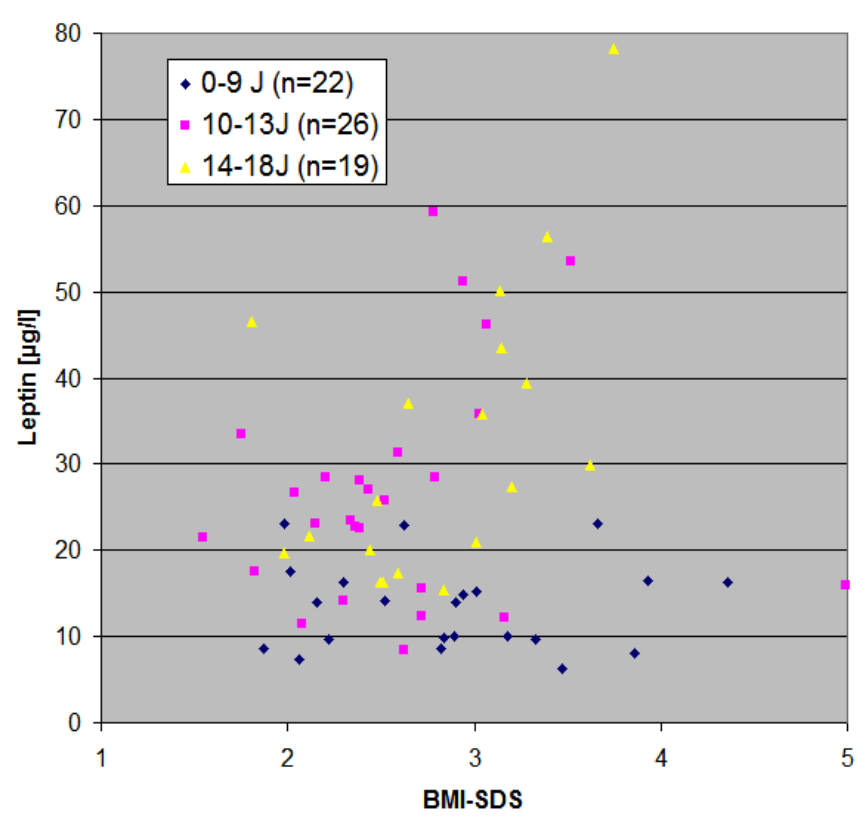

aller Jungen mit $r=0,3116$ und $p=$ 0,0055 signifikant ist.

Bei den Mädchen findet sich der deutlichste Zusammenhang zwischen Leptinspiegel und BMISDS mit $r=0,5315$ in der Gruppe der 14-18-Jährigen, der als einziger im Gegensatz zu den anderen Altersgruppen bei den Mädchen mit $p=0,0192$ signifikant ist.

Ein Leptinmangel wurde beim keinem Patienten festgestellt. 


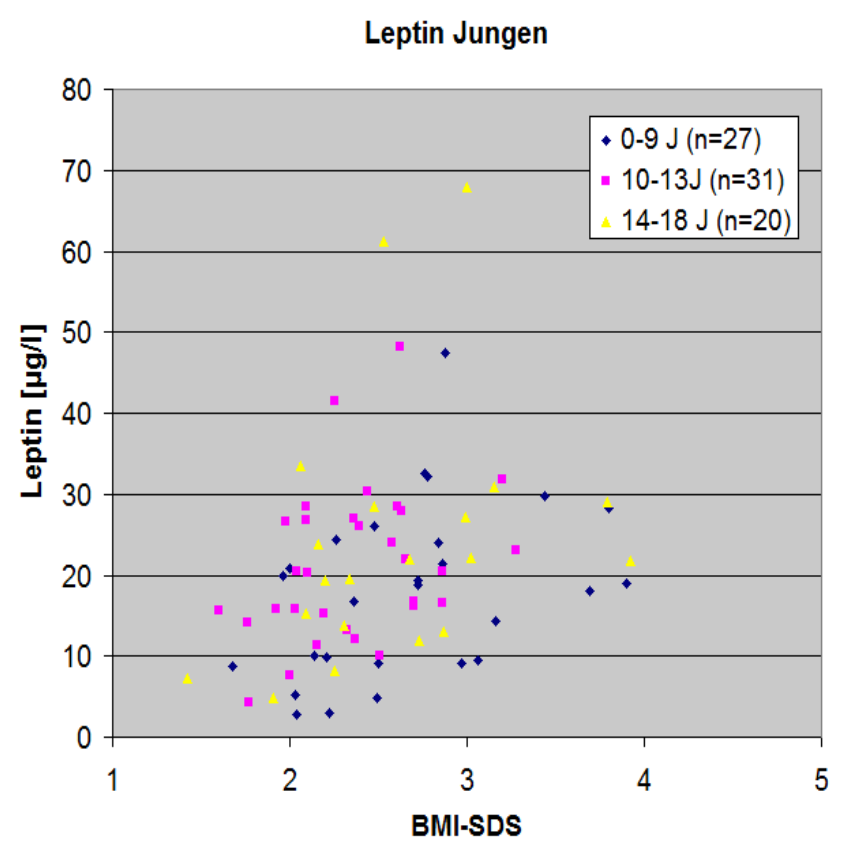

Abbildung 36a/b: Korrelation von BMI-SDS und Leptinspiegel bei Mädchen und Jungen

\subsubsection{Weitere kardiovaskuläre Risokofaktoren}

\subsubsection{Lipoprotein(a)}

Der Lipoprotein(a)-Spiegel wurde bei 135 Kindern und Jugendlichen bestimmt. 38,52\% der Werte lagen über dem oberen Grenzwert von $30 \mathrm{mg} / \mathrm{dl}$. 7,41\% sind massiv auf über das Dreifache der oberen Norm verändert.

Hierbei fanden sich, wie erwartet, weder eine Abhängigkeit vom Alter noch der Höhe des BMI-SDS.

\begin{tabular}{|c|c|c|c|c|}
\hline & $\begin{array}{l}\mathrm{Lp}(\mathrm{a}) \leq \\
30 \mathrm{mg} / \mathrm{dl}\end{array}$ & $\begin{array}{l}\mathrm{Lp}(\mathrm{a})>30 \\
\leq 60 \mathrm{mg} / \mathrm{dl}\end{array}$ & $\begin{array}{l}\mathrm{Lp}(\mathrm{a})>60 \\
\leq 90 \mathrm{mg} / \mathrm{dl}\end{array}$ & $\begin{array}{l}\text { Lp(a) > } \\
90 \mathrm{mg} / \mathrm{dl}\end{array}$ \\
\hline $\mathrm{n}$ & 83 & 26 & 16 & 10 \\
\hline$\%$ & 61,48 & 19,26 & 11,85 & 7,41 \\
\hline
\end{tabular}

Tabelle 22: Prozentuale Lp(a)-Erhöhung im Kollektiv bei Erstvorstellung

\subsubsection{Hyperhomozysteinämie}

Ein weiterer Faktor, der das kardiovaskuläre Risiko beeinflusst, ist das Homozystein. Es wurde bei 122 Mädchen und 114 Jungen bestimmt. Erfreulicherweise war dieser Wert bei keinem der Patienten erhöht. 


\subsubsection{3. Übersicht der zusätzlichen kardiovaskulären Risikofaktoren}

Zusätzlich zu den Störungen, die das metabolische Syndrom bilden, tragen weitere Faktoren, die sich nicht durch Gewichtsreduktion beeinflussen lassen, zum kardiovaskulären Gesamtrisiko der Kinder und Jugendlichen bei, wie Homozystein, Lp(a) und LDL-Cholesterin. 129 Kinder, 60 Jungen und 69 Mädchen, wurden gleichzeitig auf alle drei Parameter untersucht. Homozystein war bei allen Patieneten im Normbereich.

$30,43 \%$ der Mädchen und 43,33\% der Jungen waren frei von zusätzlichen Risikofaktoren, während bei 18,84\% der Mädchen und 15,00\% der Jungen, zusätzlich zu Übergewicht, bzw. Adipositas und deren möglichen Folgeerkrankungen, zwei weitere wichtige kardiovaskuläre Risikofaktoren bestehen.

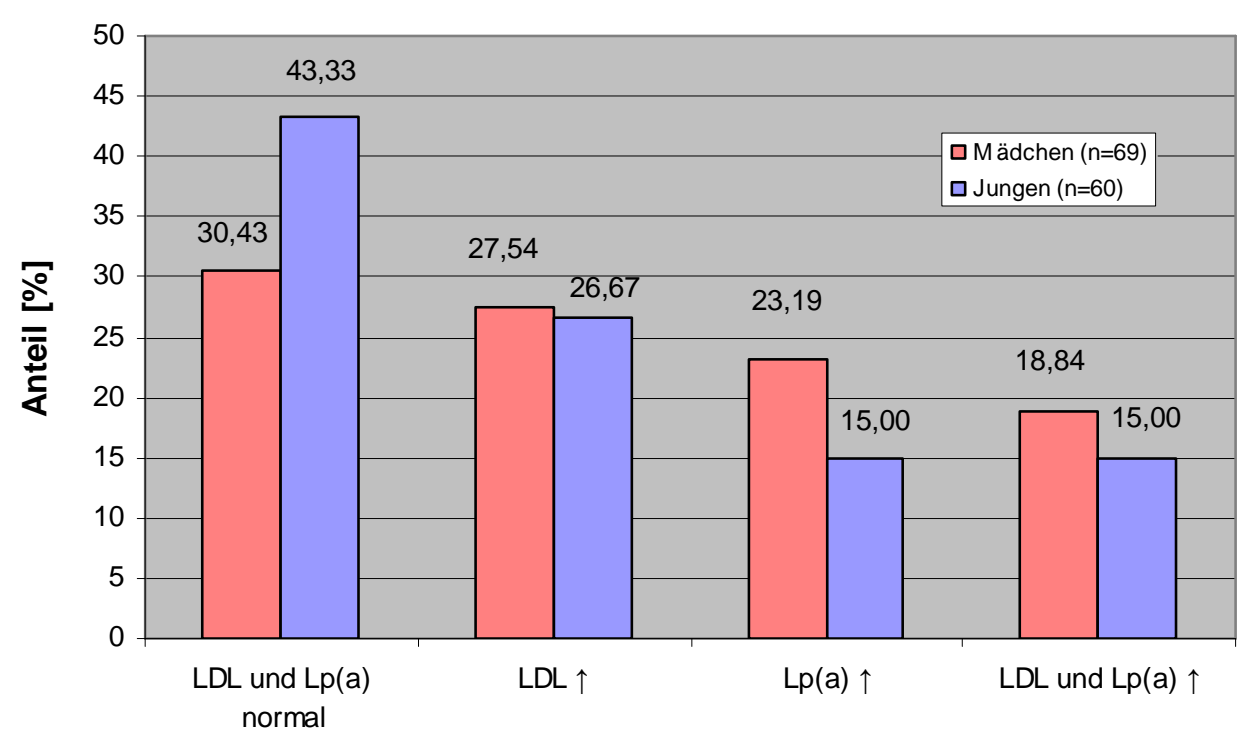

Abbildung 37: Anteil der Kinder mit Zusätzlich zum metabolischen Syndrom erhöhtem kardiovaskulären Risiko

\subsection{Gewichtsverlauf vor der Intervention}

\subsubsection{Analyse der Daten aus den Vorsorgeuntersuchungen}

Aus der Analyse der Daten aus den Vorsorgeuntersuchungen geht hervor, dass bereits ab dem Zeitpunkt der U3 in der vierten bis sechsten Lebenswoche bei Jungen und der U4 im dritten bis sechsten Lebensmonat bei den Mädchen das betrachtete Kollektiv signifikant nach oben vom 50. Perzentil nach Kromeyer-Hauschild (BMI-SDS=0) [AGA 2006] abweicht. Zur Zeit der beginnenden Mobilität der Kinder zwischen sechs und 12 Monaten (U5/ U6) wird der Unterschied zum Normalkollektiv nochmals kleiner, danach nimmt er wieder zu und wird mit steigendem Alter immer größer. Bei den Untersuchungen U8 (43.-48. Lebensmonat) und 
U9 (60.-64. Lebensmonat) ist der Median des Kollektives bei den Mädchen bereits größer als 2 (U8: 2,31, U9: 2,25), also im adipösen Bereich, und bei den Jungen etwas darunter (U8:1,76, U9: 1,83).

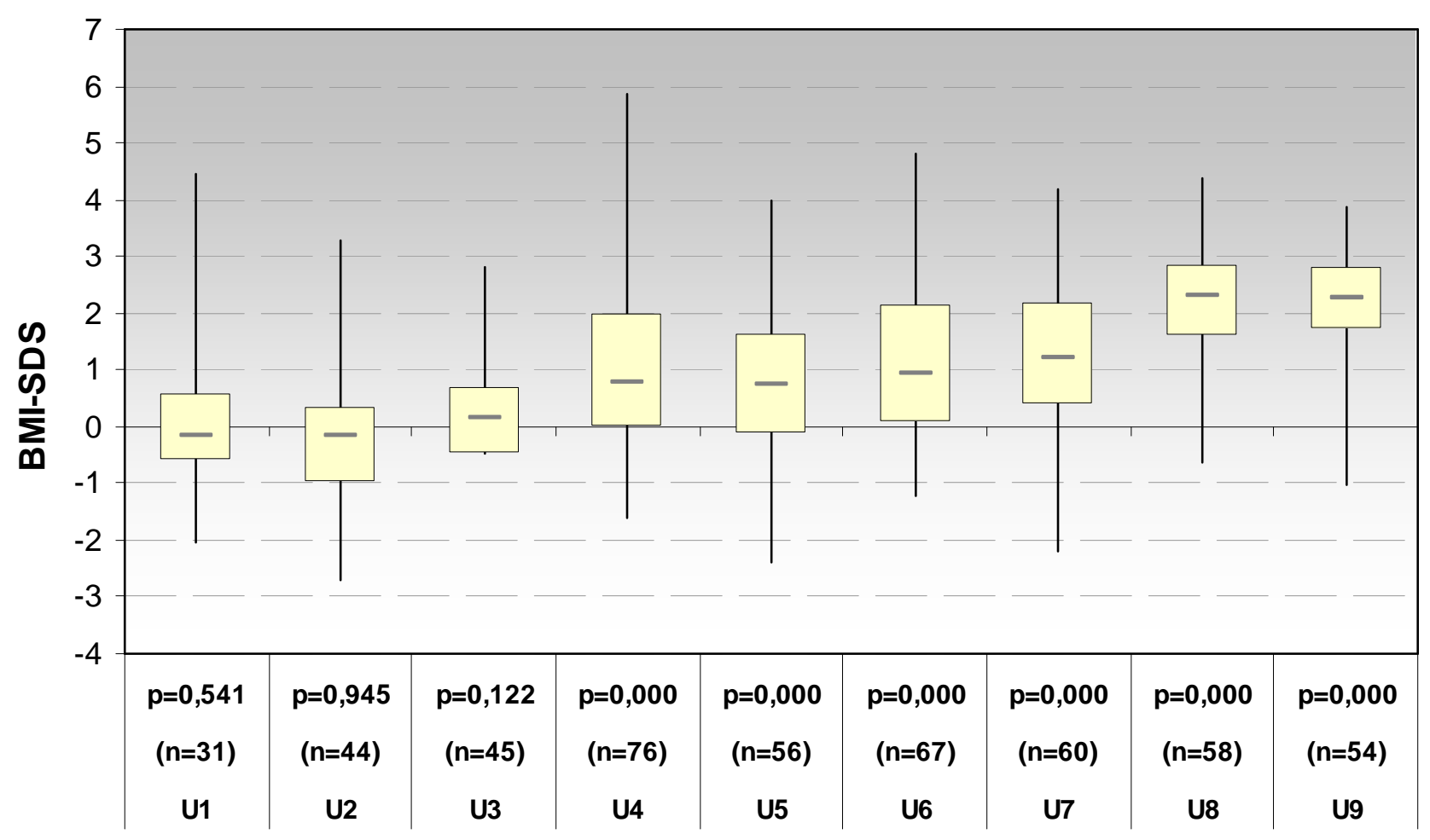

Abbildung 38: BMI-SDS-Verteilung bei den Vorsorgeuntersuchungen der Mädchen

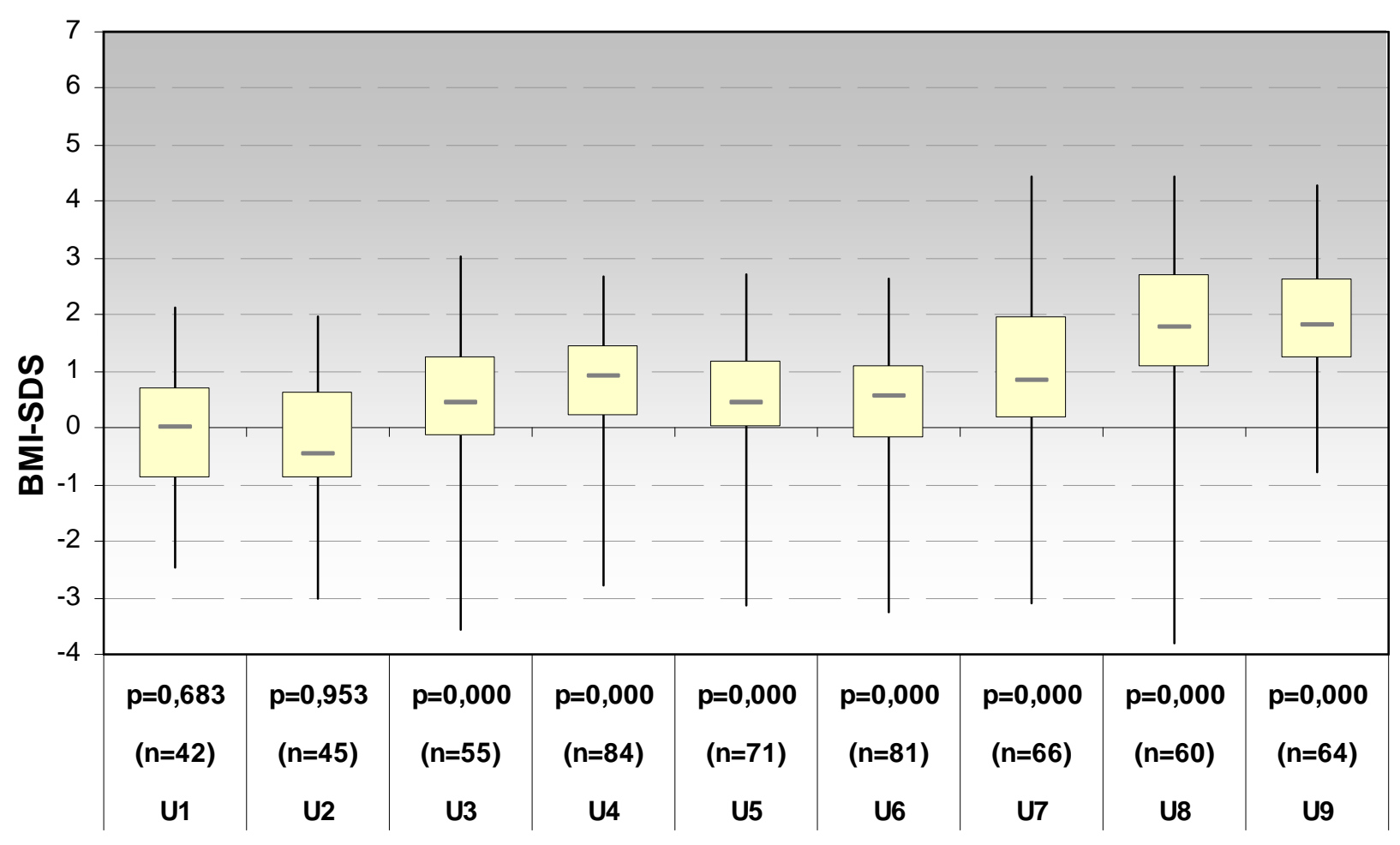

Abbildung 39: BMI-SDS-Verteilung bi den Vorsorgeuntersuchungen der Jungen 


\subsubsection{Regression des Gewichtsverlaufes vor der Intervention}

Betrachtet man den Kurvenverlauf einer polynomischen Regression des BMI-Verlaufes vor der Intervention, so schneidet diese das 97. Perzentil und somit die Grenze zur Adipositas bei den Jungen mit 4, bei den Mädchen sogar mit zwei Jahren und das 99,5. Perzentil, welches die Grenze zur extremen Adipositas ist, bei beiden Geschlechtern etwa mit 14 Jahren. Errechnet man aus der Regressionskurve den Wendepunkt, der den AR darstellt [FKE 2009], so erhält man für die Mädchen Werte bei 3,6, 7,6 und 15,7 Jahren. Die Kurve der Jungen hat rechnerisch zwei Wendepunkte bei 2,8 und 5,9 Jahren. Dem AR entsprechen hierbei nach visueller Bestimmung die Wendepunkte bei 3,6 Jahren für Mädchen und 2,8 Jahren bei den Jungen.

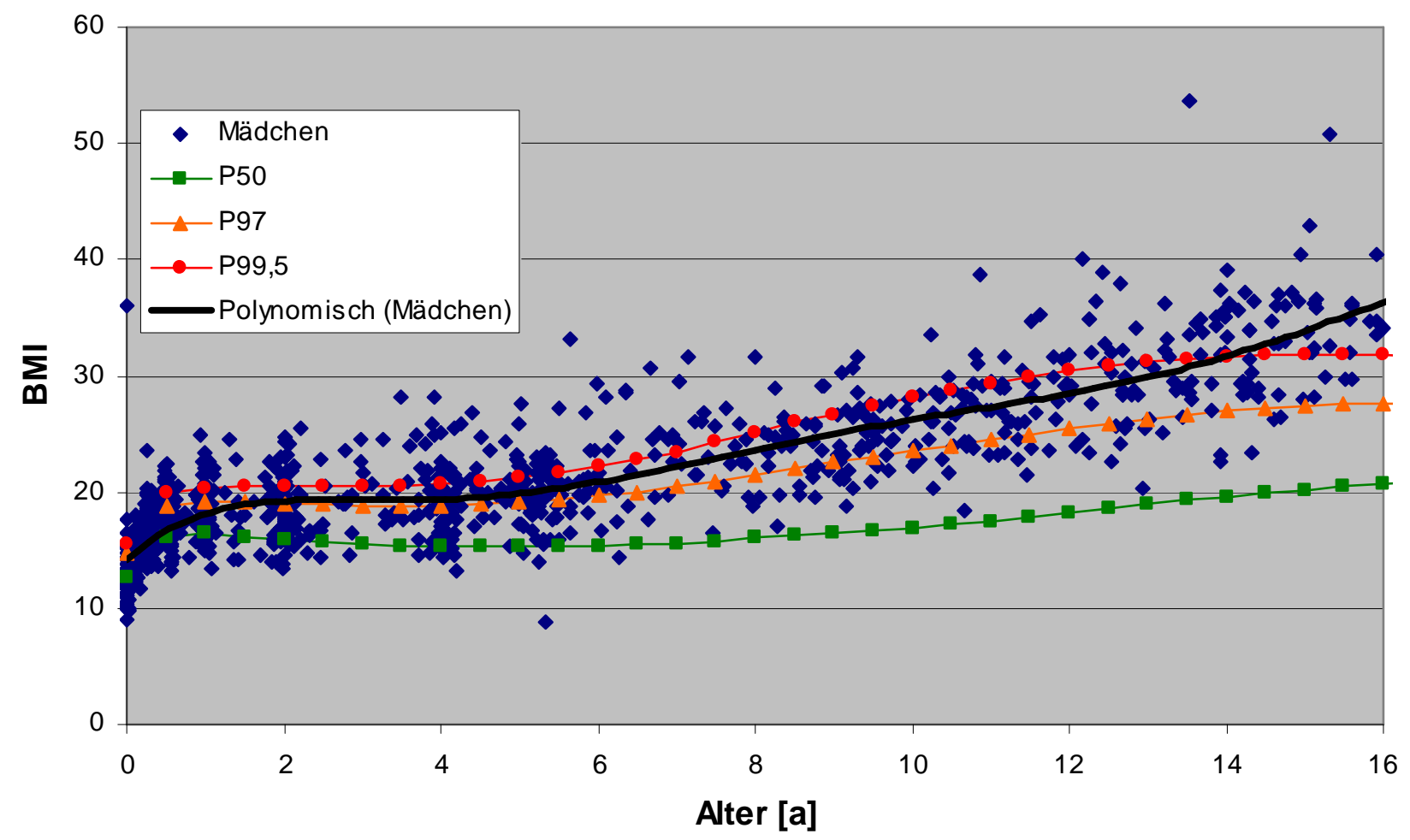

Abbildung 40: Gewichtsverlauf der Mädchen vor der Intervention 


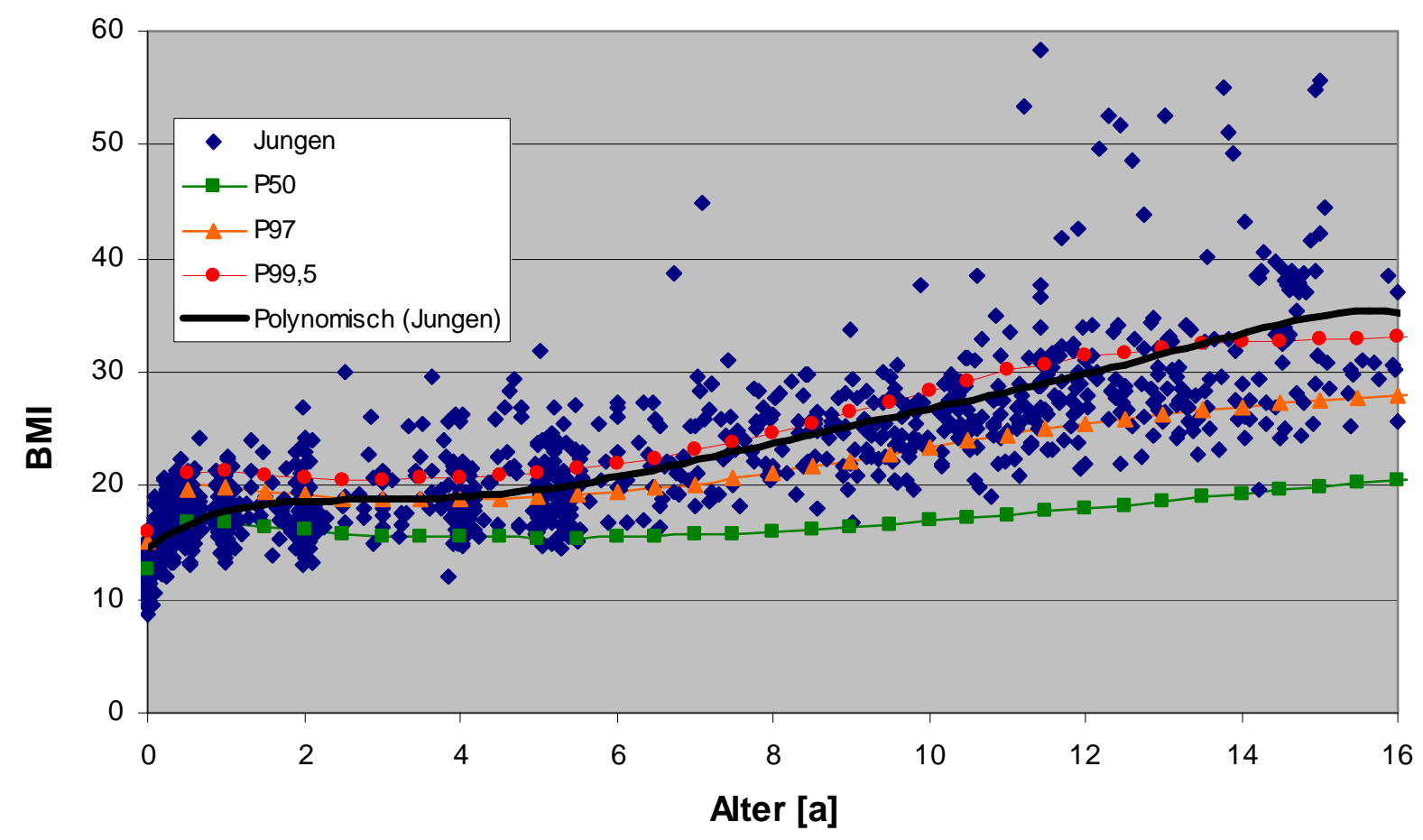

Abbildung 41: Gewichtsverlauf der Jungen vor der Intervention

\subsubsection{Zusammenhang mit Geburtsgewicht und Geburtswoche}

Ein Zusammenhang zwischen Geburtsgewicht und Ausmaß der Adipositas bei Erstvorstellung konnte weder für hypo- noch hypertrophe Neugeborene nachgewiesen werden. Der Anteil Frühgeborener betrug insgesamt 11,31\%. Die ehemals früh geborenen Kinder und Jugendlichen hatten aber keinen erhöhten BMI-SDS gegenüber denen, die ab der 37. SSW geboren wurden.

\begin{tabular}{|c|c|c|c|c|}
\hline & Jungen & & Mädchen & \\
\hline SSW & $<37$ & $\geq 37$ & $<37$ & $\geq 37$ \\
\hline $\mathrm{n}(327)$ & 17 & 161 & 20 & 129 \\
\hline$\%$ & 8,99 & 90,45 & 13,42 & 86,58 \\
\hline$\varnothing \mathrm{BMI}-S D S$ bei EV & $2,41 \pm 0,50$ & $2,483 \pm 0,59$ & $2,64 \pm 0,73$ & $2,62 \pm 0,75$ \\
\hline p t-Test & 0,61 & Ref. & 0,90 & Ref. \\
\hline
\end{tabular}

\begin{tabular}{|c|c|c|c|c|c|c|}
\hline & \multicolumn{3}{|l|}{ Jungen } & \multicolumn{3}{|l|}{ Mädchen } \\
\hline Geburtsgewich & $<2500$ & $2500-4000$ & $>4000$ & $<2500$ & $2500-4000$ & $>4000$ \\
\hline n (363) & 12 & 136 & 46 & 16 & 128 & 25 \\
\hline$\%$ & 6,19 & 70,1 & 23,71 & 9,47 & 75,74 & 14,79 \\
\hline$\varnothing \mathrm{BMI}-\mathrm{SDS}$ bei EV & $2,41 \pm 0,58$ & $2,5 \pm 0,57$ & $2,51 \pm 0,65$ & $2,31 \pm 0,59$ & $2,63 \pm 0,80$ & $2,85 \pm 0,85$ \\
\hline pt-Test & 0,32 & Ref. & 0,86 & 0,90 & Ref. & 0,45 \\
\hline
\end{tabular}

Tabelle 23: Zusammenhang zwischen Adipositas, Geburtswoche und Geburtsgewicht 


\subsection{Erfolg der Intervention}

Zur Verlaufsbeurteilung liegen nach sechs Monaten Intervention mit ärztlicher, psychologischer und/ oder ökotrophologischer Beratung die Daten von 151 (32,68\%), nach zwölf Monaten von 91 Patienten (19,70\%) vor. Nach sechs Monaten konnten $74,17 \%$ der Kinder und Jugendlichen ihren BMI-SDS im Mittel um 0,24 $\pm 0,23$ verringern. 25,38\% hatten nach sechs Monaten weiter an Gewicht zugenommen. Die mittlere Zunahme des BMI-SDS betrug bei diesen Patienten $0,11 \pm 0,10$.

Nach einem Jahr haben $74,73 \%$ der Kinder und Jugendlichen eine BMI-SDS-Reduktion erzielt (im Mittel von 0,28 $\pm 0,23$ ) und bei 25,27\% war eine Zunahme des BMI-SDS um durchschnittlich $0,17 \pm 0,18 \mathrm{zu}$ verzeichnen. Insgesamt betrachtet ist der Unterschied im BMI-SDS nach sechs Monaten durchschnittlich $-0,15 \pm 0,26$ und nach zwölf Monaten $-0,17 \pm$ 0,30, also deutlich im negativen Bereich.

Aus dem Diagramm ist ersichtlich, dass die meisten Kinder und Jugendlichen nach sechs Monaten Intervention nur eine leichte Reduktion des BMI-SDS erreichten, nach zwölf Monaten der Anteil derer, die ihren BMI-SDS um 0,2 oder sogar 0,5 reduzieren konnten, zunahm.

Im Gegensatz dazu wurde der Anteil der Patienten, die nach sechs Monaten bereits weiter zugenommen hatten, auch nach zwölf Monaten nicht mehr größer, sondern sank leicht.

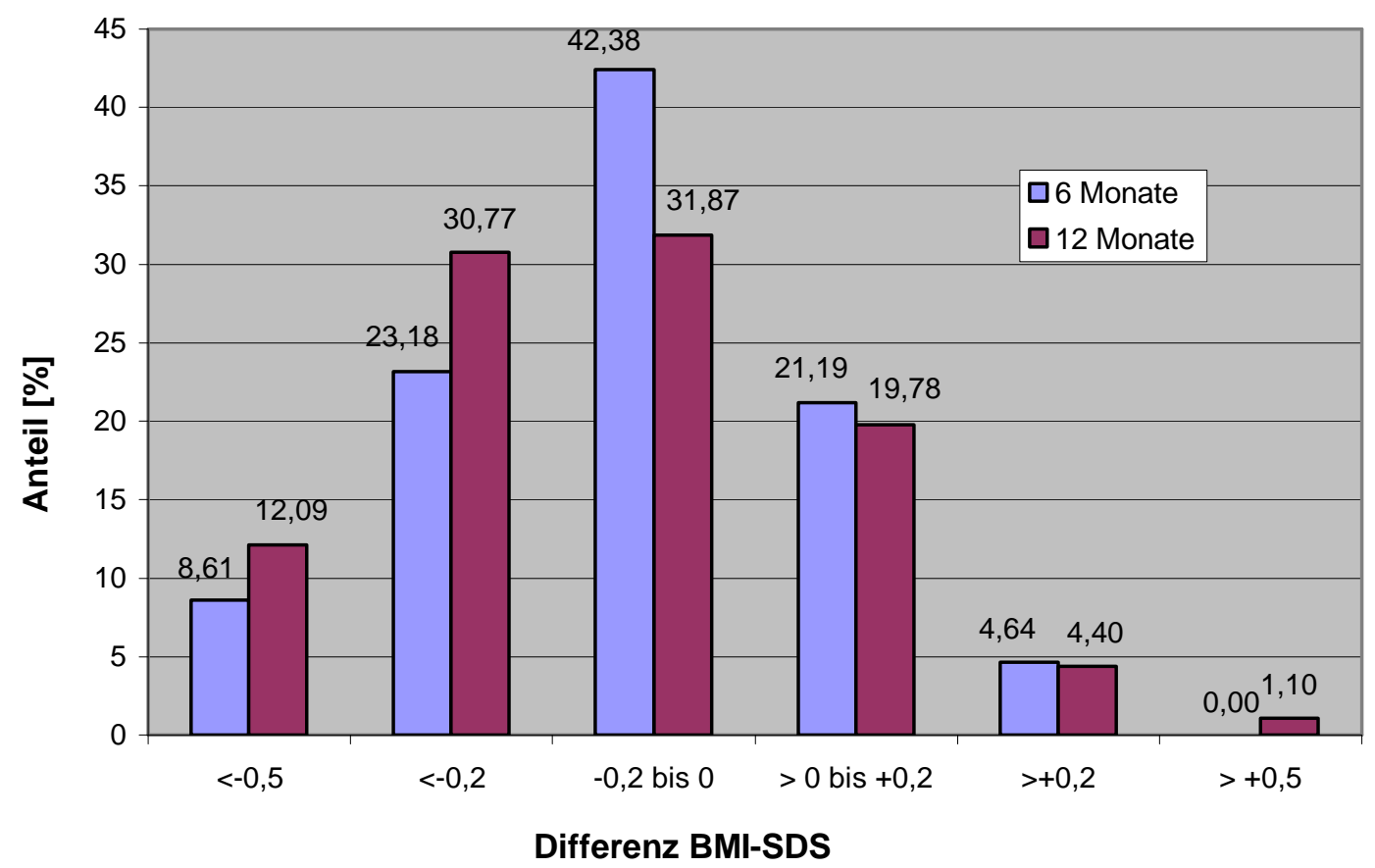

Abbildung 42: Reduktion des BMI-SDS nach sechs und zwölf Monaten

Der vollständige Verlauf mit Daten nach sechs und zwölf Monaten Intervention lag von 55 Patienten vor (Abb. 43). Aufgetragen ist die Differenz des BMI-SDS nach sechs und zwölf Monaten gegen die Differenz des BMI-SDS zu Therapiebeginn und nach sechs Monaten. 
Im linken unteren Quadranten befinden sich diejenigen 50,00 \%, die sowohl im ersten als auch im zweiten halben Jahr ihren BMI-SDS verringern konnten. Im linken oberen Quadranten die, die nach sechs Monaten abgenommen hatten, aber

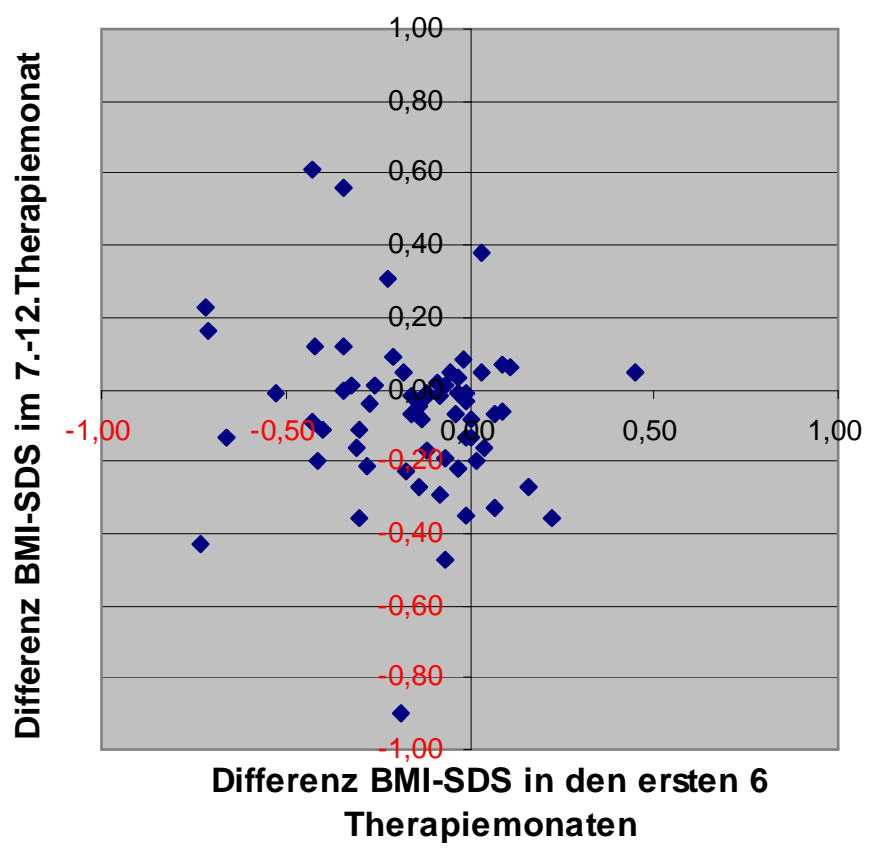
dann wieder zunahmen $(28,13 \%)$. Ein kleinerer Teil von 14,06 \% hatte initial nach sechs Monaten keine Gewichtsreduktion, nach einem Jahr aber den BMI-SDS verringert. Die kleinste Gruppe bilden die Kinder und Jugendlichen, bei denen sich sowohl nach sechs als auch nach zwölf Monaten das Ausmaß der Adipositas verschlimmert hatte, wie es bei $7,81 \%$ der Fall war.

Abbildung 43: Erfolg nach sechs und zwölf Monaten im Vergleich

Nach dem Alter betrachtet sind die Patienten, die nach sechs Monaten und nach zwölf Monaten Intervention weiter abgenommen haben, signifikant jünger als die, die sowohl nach den ersten als auch nach den zweiten sechs Monaten der Therapiedauer zugenommen haben $(p=0,021)$.

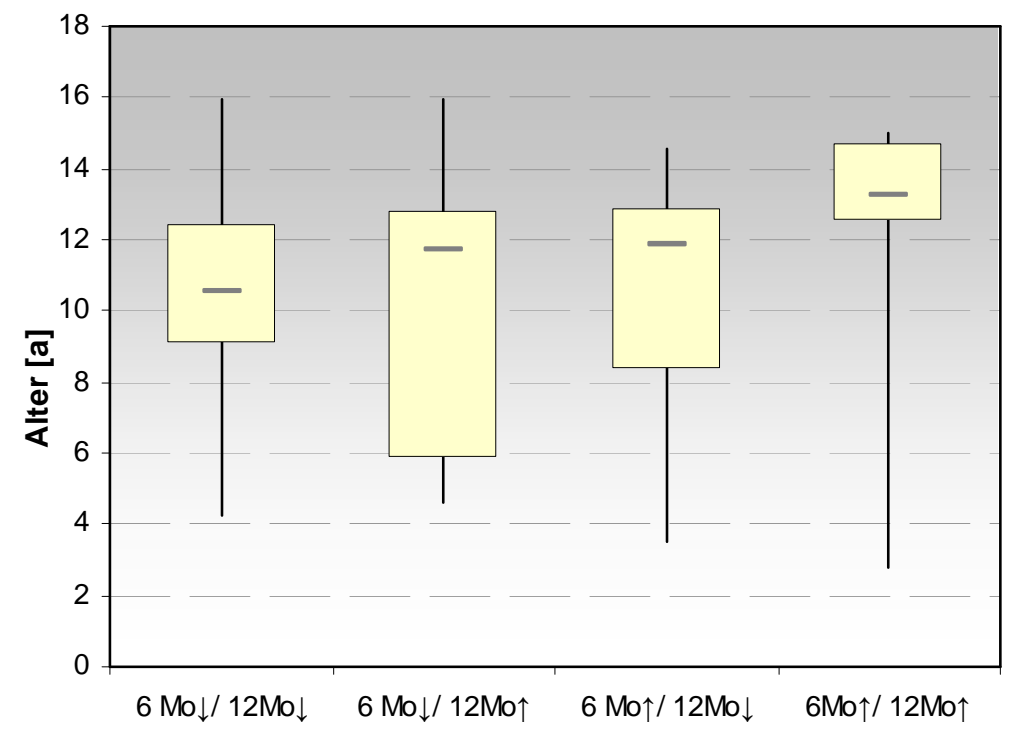

Abbildung 44: Altersverteilung nach Therapieerfolg 
Bei der Einteilung nach Alter der Kinder und Jugendlichen zu Therapiebeginn ergab sich, dass alle Gruppen den medianen BMI-SDS nach zwölf Monaten weiter reduzieren konnten als nach sechs Monaten. Die größte negative BMI-SDS-Bilanz gab es bei der Altersgruppe bis neun Jahre. Diese Gruppe hatte auch nach sechs Monaten signifikant mehr an Gewicht verloren als die Gruppe der 10- bis 13-Jährigen. Die jüngeren Kinder konnten ihren BMISDS auch stärker reduzieren als die 14-18-Jährigen, dieser Unterschied war jedoch nicht signifikant.

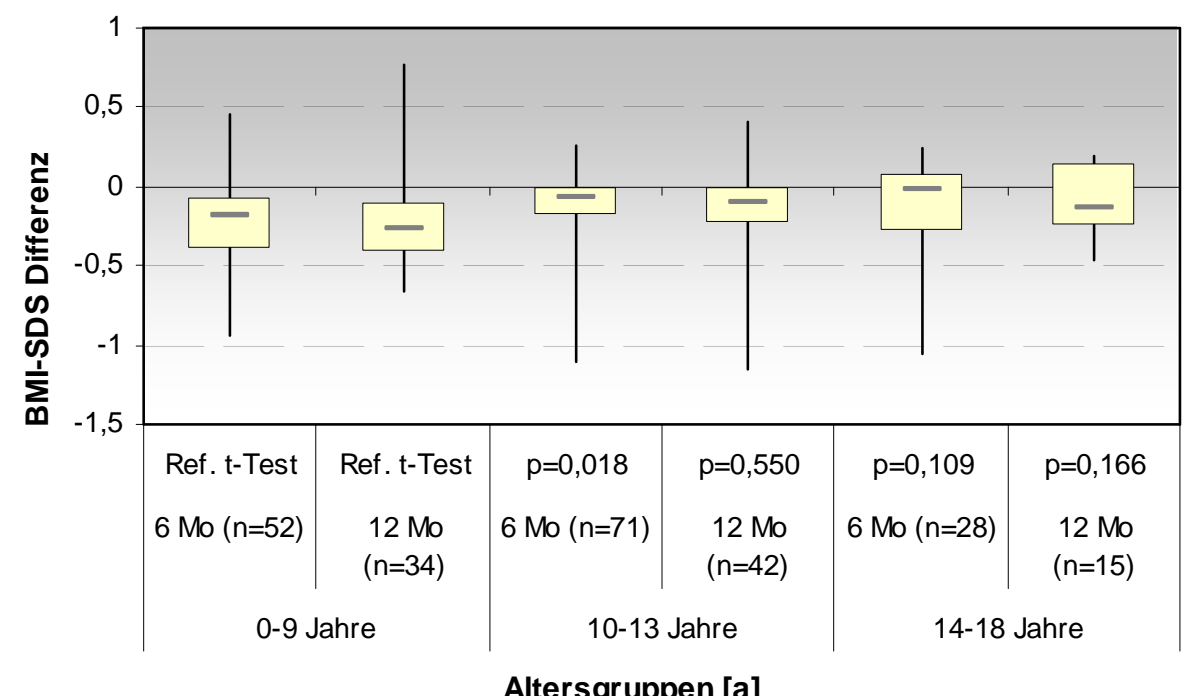

Abbildung 45: Differenz des BMI-SDS nach sechs und zwölf Monaten Intervention nach Alter

Im Vergleich zwischen den Geschlechtern sieht man, dass Jungen und Mädchen nach sechs Monaten ihren BMI-SDS etwa gleich gut reduzieren konnten, die Jungen aber auf die lange Sicht nach einem Jahr signifikant $(p=0,049)$ erfolgreicher waren. Nach einem Jahr hatten $84,62 \%$ der Jungen ihren BMI-SDS um durchschnittlich 0,28 $\pm 0,19$ reduziert. Bei 11,54\% betrug die negative Differenz sogar mehr als 0,5. Bei 15,38\% zeigte sich nach einem Jahr ein im Mittel um 0,07 $\pm 0,07$ höheren BMI-SDS.

Bei den Mädchen wurde nach einem Jahr Therapie bei nur 61,54\% eine Reduktion des BMISDS um durchschnittlich 0,30 \pm 0,31 festgestellt. 12,85\% reduzierten den Ausgangswert deutlich mit einer negativen Differenz von über 0,5. Dieser Anteil ist also ähnlich hoch wie bei den Jungen. Ein großer Anteil von 38,46\% der Mädchen hatte nach einem Jahr allerdings weiter zugenommen. Die mittlere BMI-SDS-Zunahme in dieser Gruppe betrug $0,22 \pm 0,21$. 


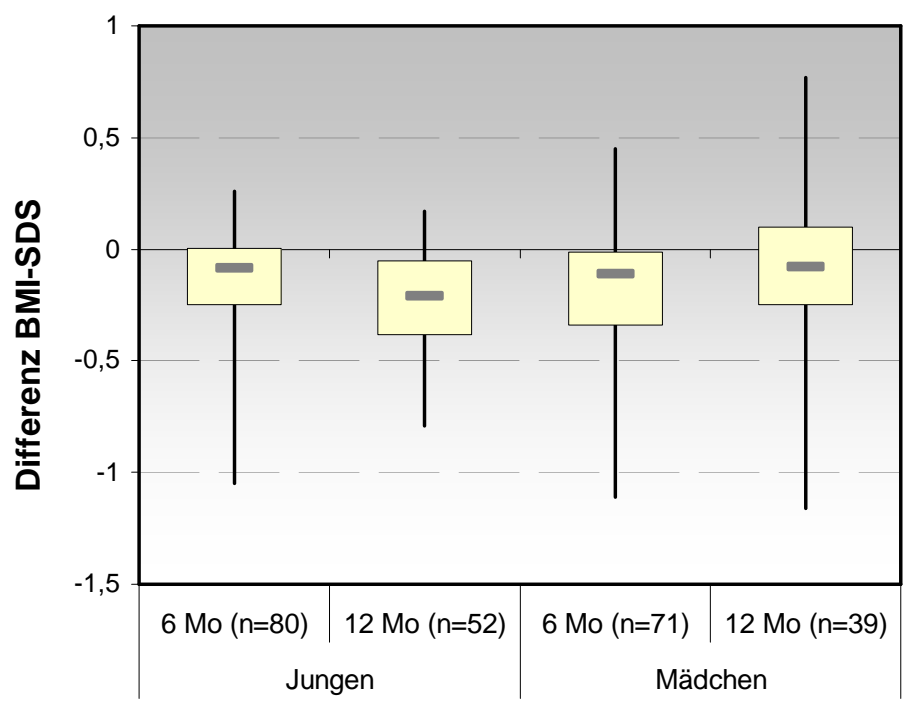

Abbildung 46: Differenz des BMI-SDS nach sechs und zwölf Monaten nach Geschlecht

Betrachtet man nun die einzelnen Altersgruppen, sieht man, dass die Jungen bis zehn Jahren mit einer negativen BMI-SDS-Differenz von 0,29 $\pm 0,17$ am meisten abgenommen haben. Den Jungen ist es im Mittel in allen Altersgruppen gelungen, im ersten und auch im zweiten Therapiehalbjahr ihren BMI-SDS zu reduzieren. Am geringsten war das Ausmaß der Reduktion bei den 14-18-jährigen Jungen.

Die mittlere BMI-SDS-Differenz nach sechs Monaten Intervention ist auch bei den Mädchen in allen Altersgruppen negativ. Meist gelang es den Patientinnen jedoch nicht, diese Erfolge im zweiten halben Jahr der Therapie fortzusetzen. Die durchschnittliche Differenz zum Ausgangs-BMI-SDS ist zwar immer noch negativ, aber im Vergleich zum Sechsmonatswert finden wir eine BMI-SDS-Zunahme in den Gruppen bis 10 Jahren und 14-18 Jahren. Bei den 10-13-jährigen Mädchen zeigt sich nach sechs und zwölf Monaten die gleiche mittlere BMI-SDS-Differenz.

\begin{tabular}{|c|c|c|c|c|c|c|}
\hline \multicolumn{7}{|l|}{ Mädchen } \\
\hline $\begin{array}{l}\text { Alter } \\
\text { Therapie }\end{array}$ & $\begin{array}{l}0-9 \text { Jahre } \\
6 \text { Monate }\end{array}$ & 12 Monate & $\begin{array}{l}\text { 10-13 Jahre } \\
6 \text { Monate }\end{array}$ & 12 Monate & $\begin{array}{l}\text { 14-18 Jahre } \\
6 \text { Monate }\end{array}$ & 12 Monate \\
\hline $\mathrm{n}$ & 33 & 13 & 27 & 20 & 11 & 6 \\
\hline $\begin{array}{l}\text { Ø Differenz } \\
\text { BMI-SDS }\end{array}$ & $-0,22 \pm 0,29$ & $-0,09 \pm 0,43$ & $-0,12 \pm 0,27$ & $-0,12 \pm 0,39$ & $-0,11 \pm 0,24$ & $-0,05 \pm 0,18$ \\
\hline
\end{tabular}

\begin{tabular}{|c|c|c|c|c|c|c|}
\hline \multicolumn{7}{|l|}{ Jungen } \\
\hline $\begin{array}{l}\text { Alter } \\
\text { Therapie }\end{array}$ & $\begin{array}{l}0-9 \text { Jahre } \\
6 \text { Monate }\end{array}$ & 12 Monate & $\begin{array}{l}\text { 10-13 Jahre } \\
6 \text { Monate }\end{array}$ & 12 Monate & $\begin{array}{l}\text { 14-18 Jahre } \\
6 \text { Monate }\end{array}$ & 12 Monate \\
\hline $\mathrm{n}$ & 27 & 21 & 38 & 22 & 15 & \\
\hline $\begin{array}{l}\varnothing \text { Differenz } \\
\text { BMI-SDS }\end{array}$ & $-0,19 \pm 0,23$ & $-0,29 \pm 0,17$ & $-0,10 \pm 0,21$ & $-0,21 \pm 0,23$ & $-0,13 \pm 0,33$ & $-0,11 \pm 0,24$ \\
\hline
\end{tabular}

Tabelle 24: Mittelwerte der Reduktion des BMI-SDS nach sechs und zwölf Monaten für Jungen und Mädchen 


\subsubsection{Therapiegruppen}

Die Patienten wurden je nach Therapiekonzept in drei Gruppen eingeordnet: Schulung nach AGA-Leitlinien, multiprofessionelles Team und Einzelberatung. Bei den Kindern, die sich mindestens sechs Monate in Therapie befanden, erhielt der mit 42,98\% größte Teil eine Einzelberatung.

Nach einem Jahr Therapiedauer befand sich fast die Hälfte der Kinder (46,75\%) in Behandlung eines multiprofessionellen Teams.

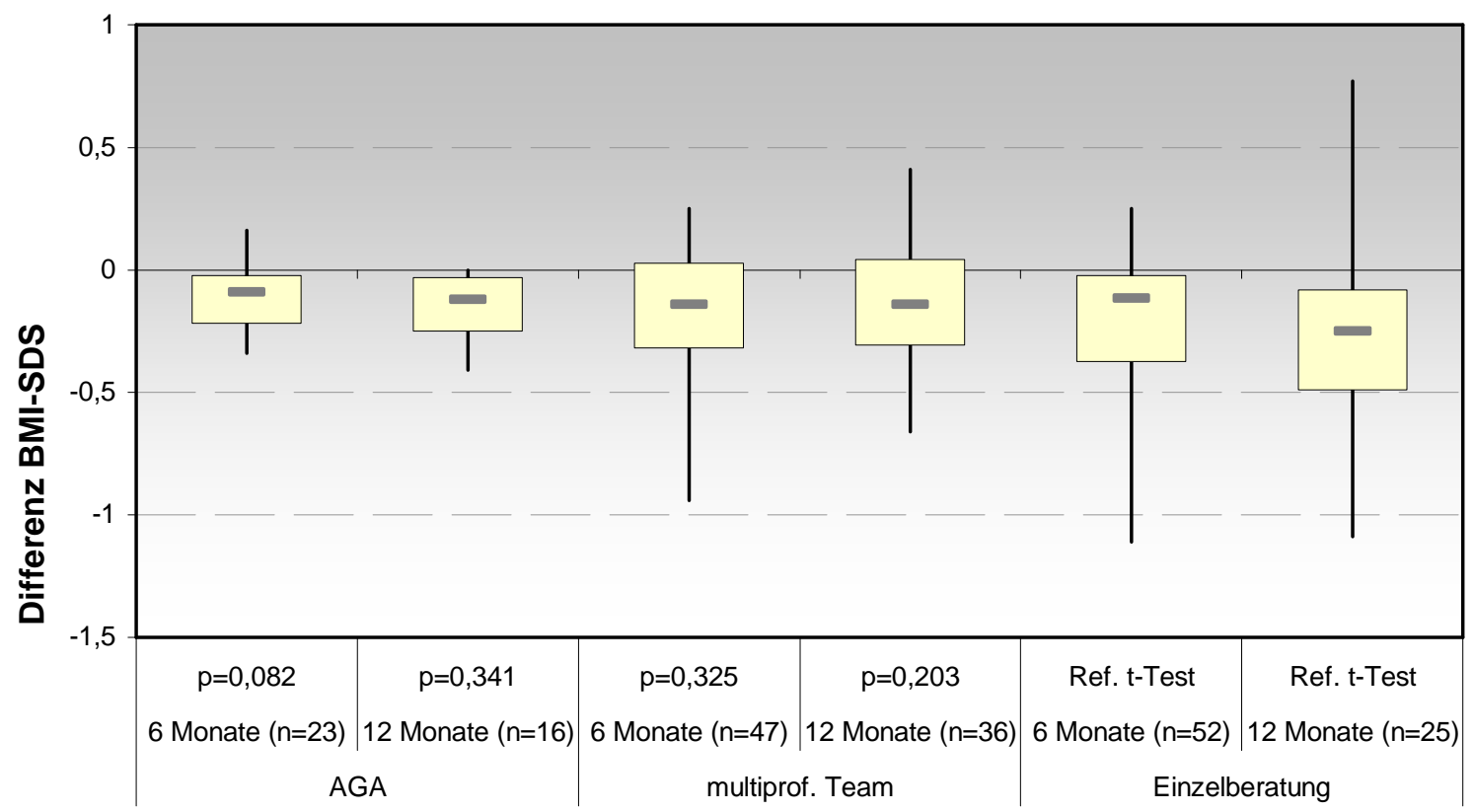

Abbildung 47: Differenz im BMI-SDS nach Therapiegruppen nach sechs und zwölf Monaten Intervention

\begin{tabular}{|l|r|r|r|}
\hline \multicolumn{2}{|l|}{ Therapiedauer 6 Monate } & \multicolumn{3}{l|}{ Multiprofessionelles Team } & Einzelberatung \\
\hline \multicolumn{1}{|c|}{ Therapie } & 23 & 46 & 52 \\
\hline $\mathrm{n}$ & 19,01 & 38,02 & 42,98 \\
\hline$\%$ & $2,53 \pm 0,31$ & $2,79 \pm 0,55$ & $2,64 \pm 0,72$ \\
\hline Ausgangs-BMI-SDS & $-0,16 \pm 0,26$ & $-0,22 \pm 0,29$ \\
\hline$\varnothing$ Differenz BMI-SDS & $-0,11 \pm 0,13$ & & \\
\hline
\end{tabular}

Therapiedauer 12 Monate

\begin{tabular}{|l|r|r|r|}
\hline Therapie & AGA & multiprofessionelles Team & Einzelberatung \\
\hline $\mathrm{n}$ & 16 & 36 & 25 \\
\hline$\%$ & 20,78 & 46,75 & 32,47 \\
\hline Ausgangs-BMI-SDS & $2,57 \pm 0,40$ & $2,85 \pm 0,61$ & $2,42 \pm 0,48$ \\
\hline$\varnothing$ Differenz BMI-SDS & $-0,14 \pm 0,12$ & $-0,13 \pm 0,25$ & $-0,24 \pm 0,38$ \\
\hline
\end{tabular}

Tabelle 25: Verteilung der Patienten in den Therapiegruppen und durchschnittliche Reduktion des $B M I-S D S$ nach sechs und zwölf Monaten Intervention 
Im Mittel lag der Ausgangs-BMI-SDS bei den Kindern und Jugendlichen, die von einem multiprofessionellen Team behandelt wurden, am höchsten. Den besten Therapieerfolg erzielten die Patienten in Einzelberatung mit einer BMI-SDS-Differenz von -0,22 $\pm 0,29$ nach sechs und -0,24 $\pm 0,38$ nach zwölf Monaten, der Unterschied zu den anderen Gruppen war jedoch nicht signifikant.

\subsubsection{Psychosoziale Faktoren}

\subsubsection{Migrationshintergrund}

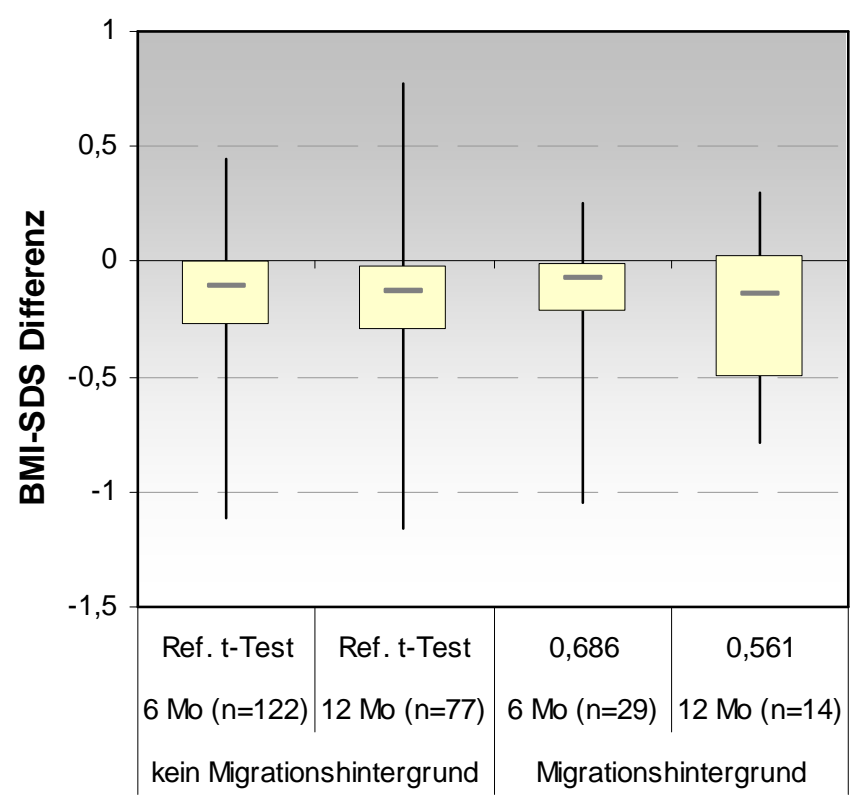

Bei der Auswertung der Unterschiede zwischen Kindern und Jugendlichen aus Familien mit und ohne Migrationshintergrund zeigte sich für unser Kollektiv, besonders nach sechs Monaten, kein relevanter Unterschied im Therapieerfolg. Der Anteil der Patienten mit Migrationshintergrund betrug bei Erstvorstellung 15,70\%, nach sechs Monaten 19,21\% und nach zwölf Monaten Intervention nur noch $15,38 \%$.

Abbildung 48: Differenz des BMI-SDS mit und ohne Migrationshintergrund nach sechs und zwölf Monaten Intervention

Die Migrantenkinder konnten ihren BMI-SDS vom Beginn der Therapie innerhalb eines Jahres um durchschnittlich 0,21 $\pm 0,32$ senken, während diese Differenz bei den Kindern und Jugendlichen ohne Migrationshintergrund lediglich $0,17 \pm 0,31$ betrug. Doch dieser Unterschied war nicht signifikant $(p=0,561)$.

\subsubsection{Ausbildung und Berufstätigkeit der Eltern}

Es konnten keine signifikanten Unterschiede im Therapieerfolg der Patienten bei einer Auswertung nach Schulabschluss oder Berufstätigkeit der Mütter und Väter gezeigt werden. Auffällig war der Trend, dass die Kinder von Müttern ohne oder mit niedrigem Schulabschluss sich tendenziell auch nach einem Jahr noch in Behandlung des SPZ 
befanden und dass die Kinder voll berufstätiger Mütter nach einem Jahr mit durchschnittlich 0,314 $\pm 0,26$ mehr abgenommen hatten, als die Kinder von Hausfrauen oder geringfügig beschäftigen Müttern mit $-0,209 \pm 0,32$. Dieser Unterschied war jedoch auch nicht signifikant $(p=0,272)$.

\subsubsection{Weiterführende Schulbildung der Kinder und Jugendlichen}

Untersucht man die Gewichtsreduktion im Bezug auf die eigene Schulbildung der Patienten, stellt man fest, dass Jugendliche, die das Gymnasium besuchen, in unserem Kollektiv nach sechs und zwölf Monaten Intervention das beste Outcome haben. Mit Ausnahme der Hauptschüler schaffen es alle Gruppen im zweiten halben Jahr der Therapie den mittleren und medianen BMI-SDS weiterhin zu senken.

Hauptschüler konnten ihren medianen BMI-SDS nach sechs Monaten leicht $(-0,02)$ reduzieren, nach einem Jahr war der Median aber wieder zu seinem Ausgangswert zurückgekehrt. In dieser Gruppe liegen Median und Mittelwert durch einige Ausreißer mit einer erfreulicheren Gewichtsentwicklung weit auseinander. Die mittlere BMI-SDSReduktion liegt hier bei $-0,11 \pm 0,31$ nach sechs und $-0,12 \pm 0,39$ nach zwölf Monaten Interventionsdauer.

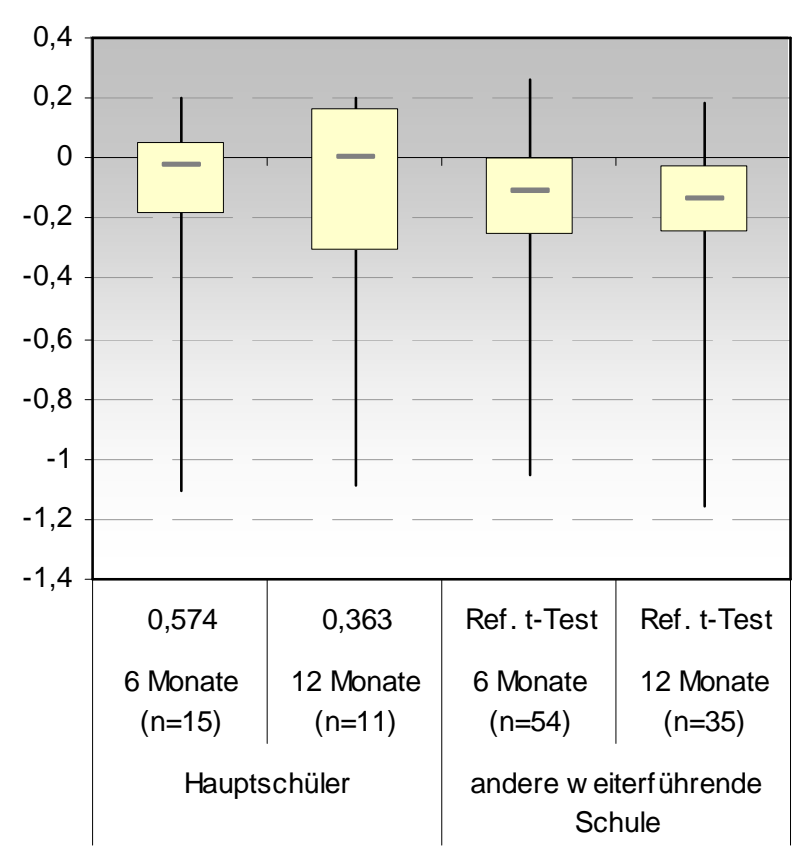

Abbildung 49: Differenz das BMI-SDS der Hauptschüler im Vergleich nach sechs und zwölf Monaten Intervention 


\begin{tabular}{|c|c|c|c|c|}
\hline & Hauptschule & Realschule & Gymnasium & Gesamtschule \\
\hline $\mathrm{N}$ & 15 & 25 & 17 & 12 \\
\hline$\varnothing$ Differenz BMI-SDS & $-0,11 \pm 0,31$ & $-0,13 \pm 0,25$ & $-0,20 \pm 0,31$ & $-0,16 \pm 0,27$ \\
\hline Median Differenz BMI-SDS & $-0,02$ & $-0,11$ & $-0,12$ & $-0,09$ \\
\hline pt-Test & 0,393 & 0,395 & Ref. & 0,450 \\
\hline
\end{tabular}

\begin{tabular}{|c|c|c|c|c|}
\hline \multicolumn{5}{|l|}{ Therapiedauer 12 Monate } \\
\hline & Hauptschule & Realschule & Gymnasium & Gesamtschule \\
\hline $\mathrm{n}$ & 11 & 16 & 12 & 7 \\
\hline$\varnothing$ Differenz BMI-SDS & $-0,12 \pm 0,39$ & $-0,19 \pm 0,22$ & $-0,27 \pm 0,31$ & $-0,16 \pm 0,27$ \\
\hline Median Differenz BMI-SDS & 0 & $-0,17$ & $-0,18$ & $-0,11$ \\
\hline p t-Test & 0,340 & 0,513 & Ref. & 0,483 \\
\hline
\end{tabular}

Tabelle 26: Durchschnittliche Differenz des BMI-SDS nach weiterführender Schulbildung nach sechs und zwölf Monaten Intervention 


\section{Diskussion}

\subsection{Status bei Erstvorstellung}

\subsubsection{Biometrische Daten}

Im untersuchten Kollektiv war der Anteil an Mädchen und Jungen bei Erstvorstellung etwa gleich groß. Die meisten Patienten stellten sich im Schulkindalter zwischen sieben und 14 Jahren erstmals in der Adipositasambulanz vor. Bei den Jungen waren mehr als die Hälfte adipös mit einem BMI zwischen dem 97. und 99,5. Perzentil, bei den Mädchen litt die größte Gruppe (48,86\%) bei Erstvorstellung bereits an extremer Adipositas (BMl> 99,5. Perzentil). Jungen stellten sich hierbei sowohl in Bezug auf das Alter als auch auf den BMI-SDS früher vor als die Mädchen. Eine mögliche Erklärung wäre die höhere Rate an meist internalisierenden Verhaltensproblemen bei Mädchen [Warschburger 2006], durch welche die Gewichtsproblematik eventuell schon in der Familien schwerer zu thematisieren und die Hemmschwelle zur Hilfesuche erhöht sein könnte.

Auffällig ist auch, dass die Kinder zwischen sieben und 14 Jahren, die bei Erstvorstellung die größte Gruppe bilden, im Mittel am wenigsten stark betroffen sind. Kleine Kinder zwischen null und sechs Jahren müssen demnach deutlich adipöser sein, damit adäquate Hilfe aufgesucht wird. Das unterstützt die Aussage, dass Übergewicht, besonders bei kleinen Kindern, oft verniedlicht und verharmlost wird, insbesondere, wenn die Mutter selbst übergewichtig oder adipös ist [Watkins et al. 2007].

Teenager von 14 bis 18 Jahren sind im Mittel bei Erstvorstellung deutlich schwerer von Adipositas betroffen als die jüngeren Schulkinder. Dazu passt die Beobachtung aus der KiGGS-Studie, dass die Mädchen der Altersgruppe zwischen 14-17 Jahren sich insgesamt am wenigsten bewegen [Dordel 2007, Lampert et al. 2007a, Hampson et al. 2007] und die Jungen sich am ungesündesten ernähren [Mensink et al. 2005]. Aufgrund der höheren

Beeinträchtigung des Selbstwertes und Selbstkonzeptes, besonders im Bezug auf körperliche Attraktivität und athletische Kompetenz der älteren Jugendlichen [Warschburger 2006], kann man davon ausgehen, dass viele Betroffene, gerade dieser Altersgruppe, die Hilfesuche aus Scham scheuen. 


\subsubsection{Psychosoziale Faktoren}

\subsubsection{Charakterisierung der Familien}

In der Literatur werden Kinder und Jugendliche aus Familien mit allein erziehendem Elternteil oder geringer Familienbindung als gefährdet für die Entwicklung einer Adipositas beschrieben [Röbl et al. 2006].

Laut Statistischem Bundesamt waren 2007 in Deutschland 73,08\% der Familien Ehepaare und 7,9\% Lebensgemeinschaften mit Kindern. Leider lässt sich hierbei nicht sagen, ob es sich um leibliche Kinder handelt. Der Anteil Alleinerziehender war mit 18,3\% ähnlich hoch wie in unserem Kollektiv [Statistisches Bundesamt 2008].

Auch waren Kinder und Jugendliche aus klassischen Familien mit beiden leiblichen Eltern im Kollektiv im Mittel genauso stark betroffen wie alle anderen. In unserem Kollektiv stellte also Alleinerziehenden-Status der Eltern keinen relevanten Risikofaktor dar.

Einzelkinder sind im Vergleich zum bundesdeutschen Durchschnitt seltener, aber schwerer von Adipositas betroffen als Kinder und Jugendliche mit bis zu zwei Geschwistern. Der Anteil an Familien mit zwei Kindern ist im Kollektiv etwas größer als in Gesamtdeutschland. Diese Kinder haben den geringsten durchschnittlichen BMI-SDS im Kollektiv. Kinderreiche Familien kommen im Kollektiv sehr viel häufiger vor als in Deutschland insgesamt. Kinder und Jugendliche aus Familien mit vier oder mehr Geschwistern haben im Mittel auch einen höheren mittleren BMI-SDS als diejenigen mit weniger Geschwistern. Dieser liegt etwa im gleichen Bereich wie der der Einzelkinder.

\begin{tabular}{|l|r|r|r|}
\hline n Kinder in der Familie & 1 & 2 & $\geq 3$ \\
\hline Kollektiv [\%] & 20,0 & 45,4 & 34,6 \\
\hline Stat. Bundesamt 2007 [\%] & 52,6 & 36,6 & 10,8 \\
\hline
\end{tabular}

Tabelle 27: Vergleich des Anteils kinderreicher Familien im Kollektiv und in Gesamtdeutschland

Laut Statistischem Bundesamt waren 2005 Alleinerziehende und kinderreiche Familien weit häufiger von Armut bedroht als Familien mit zwei Erwachsenen und weniger als drei Kindern [Stat. Bundesamt 2008], was wiederum mit einem erhöhten Adipositasrisiko einhergeht [Kurth und Schaffrath-Rosario 2007].

\subsubsection{Migrationshintergrund}

In der KiGGS-Studie wurde ein deutschlandweiter Anteil an Kindern und Jugendlichen mit Migrationshintergrund von 13,4\% ermittelt, in der Stadt Göttingen hatten 2008 18,5\% der 
Einwohner einen Migrationshintergrund [Gösis 2010]. Der Migrantenanteil des untersuchten Kollektivs liegt mit 15,7\% zwischen diesen beiden Zahlen. Für Göttingen ist also der Anteil an Migrantenkindern im Kollektiv nicht erhöht. Die Kinder und Jugendlichen sind etwas, aber nicht signifikant, stärker von Adipositas betroffen als die Kinder ohne Migrationshintergrund. In anderen Studien, wie der KiGGS-Studie, wurde Migrationshintergrund als Risikofaktor für Adipositas identifiziert [Kurth und Schaffrath-Rosario 2007]. In der Adipositassprechstunde des Sozialpädiatrischen Zentrums der Charité Berlin betrug der Anteil an Migrantenkindern mit 50,15\% sogar knapp über die Hälfte [Meinlschmidt 2008].

Nach Einschätzung des Integrationsbeauftragten des Landkreises Göttingen Isa Sandiraz fehlt im Göttinger Migrantenkollektiv das klassische Gastarbeitermilieu. Ein großer Anteil der Bürger mit Migrationshintergrund seien entweder Akademiker oder geduldete Flüchtlinge, die wenig Gesundheitsleistungen in Anspruch nehmen, um nicht unnötig auf sich aufmerksam zu machen [Sandiraz 2010]. Diese Tatsachen könnten erklären, warum in dieser Untersuchung kein erhöhtes Adipositasrisiko bei Migrantenkindern gezeigt werden konnte.

\subsubsection{BMI der Eltern}

Dass dicke Eltern dicke Kinder haben, konnte schon in viele Studien mit großen Stichproben, wie der KiGGS-Studie, gezeigt werden [Röbl et al. 2008, Kurth und Schaffrath-Rosario 2007]. Auch in unserem Kollektiv bestätigte sich diese Beobachtung: Der Anteil an adipösen Männern und Frauen war unter den Eltern des Kollektivs deutlich höher als im bundesweiten Vergleich [Mensink et al. 2005].

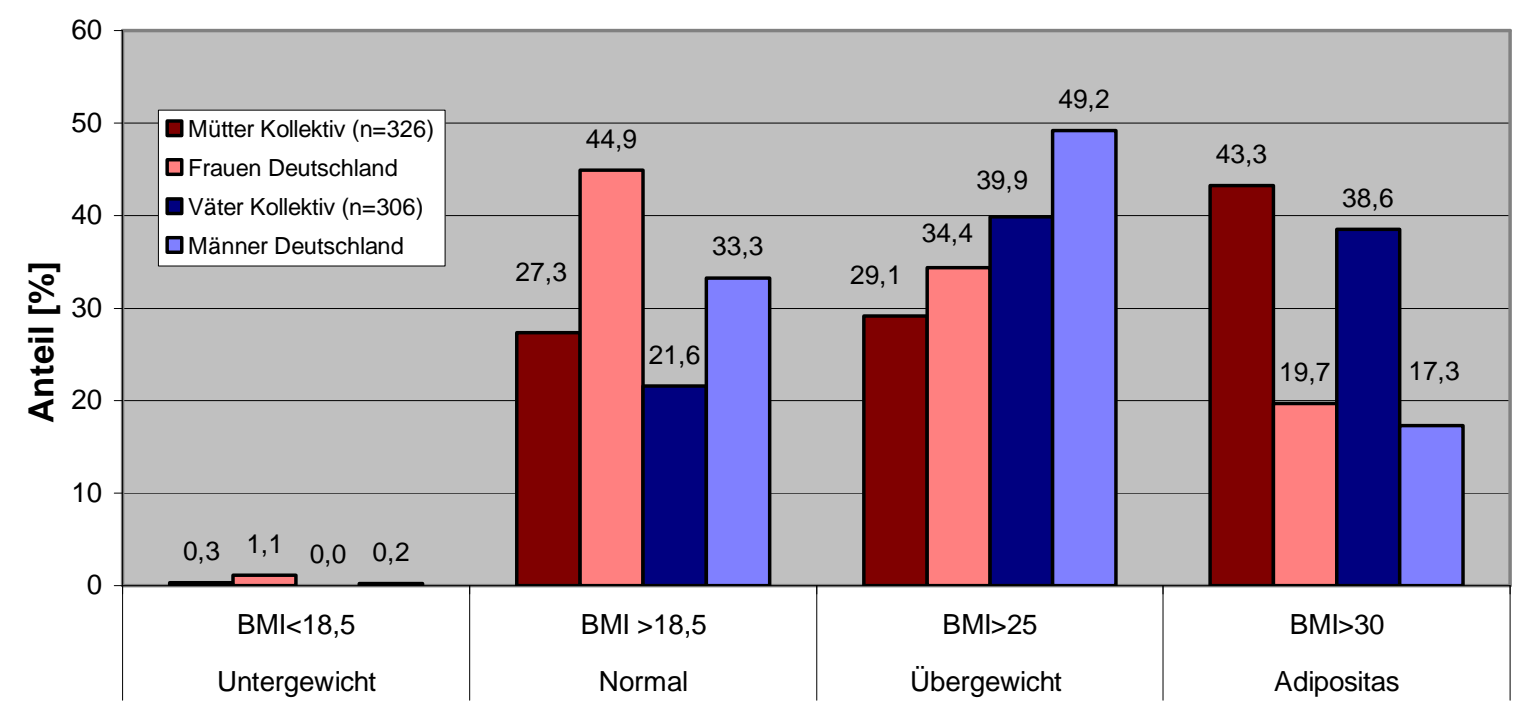

Abbildung 50: BMI der Mütter des Kollektivs im gesamtdeutschen Vergleich 
Mit dem mütterlichen BMI stieg der mediane BMI-SDS ihrer Kinder immer weiter an.

Die Kinder und Jugendlichen, deren Mütter einen BMI > $25 \mathrm{~kg} / \mathrm{m}^{2}$ hatten, waren signifikant dicker als die Kinder normalgewichtiger Mütter. Ein Einfluss des väterlichen BMI ist erst ab $35 \mathrm{~kg} / \mathrm{m}^{2}$ erkennbar, dafür aber umso deutlicher.

In der linearen Regression des BMI-SDS der Patienten mit dem ihrer Eltern ist die Korrelation in allen Fällen positiv und für Mütter und Väter mit ihren Kindern signifikant. Die stärkste Korrelation zeigte sich bei Müttern mit ihren Söhnen. Insgesamt ist die Korrelation nicht so stark wie erwartet, was unter anderem dadurch erklärt werden kann, dass die Gewichtsdaten der Eltern, besonders aber der Väter, anamnestisch erhoben wurden und übergewichtige und adipöse Menschen oft ihre eigene Größe über- und das eigene Gewicht unterschätzen [Röbl et al. 2008, Mensink et al. 2005].

Röbl et al. analysierten 2008 für 11074 Kinder die Korrelation des kindlichen mit dem mütterlichen $(r=0,29)$ und dem väterlichen BMI-SDS $(r=0,18)$.

Somit ergab sich in dieser Doktorarbeit mit $r=0,234$ ein nicht so starker Zusammenhang für die Mütter, aber ein stärkerer Zusammenhang für die Väter $(r=0,228)$.

\subsubsection{Ausbildung und Berufstätigkeit der Eltern}

Kinder von Eltern mit Abitur mit oder ohne Hochschulabschluss sind seltener und weniger stark von Übergewicht und Adipositas betroffen. Hierbei nimmt der Schulabschluss der Mütter stärkeren Einfluss als der Abschluss der Väter.

Die Kinder und Jugendlichen des untersuchten Kollektivs haben häufiger arbeitslose oder gering verdienende Eltern als der Durchschnitt. 2008 betrug die Arbeitslosenquote in Göttingen 11,8\% [Gösis 2010]. Von den Vätern des Kollektivs waren 23,97\% arbeitslos oder Geringverdiener. Der Anteil der arbeitslosen Mütter lässt sich schwieriger ermitteln, da sich unter den 43,24\% anamnestischen „Hausfrauen“ auch Arbeitslose verbergen können.

Das mediane Ausmaß der Adipositas war in allen Gruppen etwa gleich, wobei die Kinder berufstätiger Mütter etwas schlanker waren als die von Hausfrauen.

\subsubsection{Schulbildung der Kinder und Jugendlichen}

In der Stadt Göttingen besuchten im November 200811.078 Kinder und Jugendliche eine weiterführende Schule. Davon gingen 5,46\% auf die Haupt-, 11,60\% auf die Real- und $25,61 \%$ auf die Gesamtschule. Ein ungewöhnlich hoher Anteil von 57,13\% besuchte ein Gymnasium [Gösis 2010]. Im Vergleich dazu ist der Anteil der Hauptschüler im Kollektiv mit $24,45 \%$ stark erhöht und der der Gymnasiasten eher als gering zu bewerten. Die 
Hauptschüler waren bei Erstvorstellung auch stärker betroffen als die Schüler anderer Schulen. Der Besuch einer Hauptschule kann für das untersuchte Kollektiv somit als Risikofaktor für Adipositas gewertet werden.

\subsubsection{Begleiterkrankungen}

\subsubsection{Metabolisches Syndrom}

\subsection{Insulinresistenz und Störungen der Glukosetoleranz}

Ein erhöhter HOMA-IR zeigt sich, bezogen auf das Gesamtkollektiv, in über $30 \%$ der Fälle. Ein mit dem Alter zunehmender Anteil fällt besonders bei den Mädchen auf. Bei den Jungen ist auch schon in der Altersgruppe null bis neun Jahre mit über $40 \%$ eine große Zahl betroffen. Die Patienten mit pathologischem HOMA-IR haben in der Regel einen höheren medianen BMI-SDS als die restlichen Kinder und Jugendlichen, was bei den Jungen noch etwas deutlicher ausgeprägt ist. Bei den Mädchen spielt somit der Einfluss des Alters, bei den Jungen der des BMI-SDS eine etwas stärkere Rolle. Caceres et al. (2008) fanden in einem Kollektiv von 61 adipösen Kindern und Jugendlichen eine ähnlich hohe Rate von insgesamt $39,4 \%$ bei einem HOMA-IR Cut-Off von 3,5, wobei hier auch die Jungen mit $50 \%$ häufiger betroffen waren als die Mädchen mit 39\%. Die Tatsache, dass während der Pubertät die Glukosetoleranz physiologischerweise abnimmt, deutet darauf hin, dass eine Evaluation des HOMA-IR nach einem festen Cut-Off-Wert während der Geschlechtsentwicklung nicht aussagekräftig ist. Besonders bei Mädchen im TannerStadium 2 und 3 steigen die Werte für den HOMA-IR auch bei gesunden Jungendlichen stark an [d'Annunzio et al. 2008].

Es müssen Perzentile verwendet werden, die diesen Umstand in Betracht ziehen. In dieser Studie wurden alters- und geschlechtsspezifische Perzentile verwendet, die in etwa den Pubertätsstadien entsprechen (0-9 Jahre präpubertär, 10-13 Jahre pubertär, 14-18 Jahre postpubertär) [Allard et al. 2003]. Eine genauere Auswertung würde die Auswertung nach dem Tanner-Stadium liefern [d'Annunzio et al. 2008], das leider in unserem Kollektiv unvollständig erhoben wurde. Nach Caceres et al. (2008) wäre die Prävalenz der Insulinresistenz in unserem Kollektiv mit 32,37\% geringer als nach Allard et al. (38,24\%). Da nach d'Annunzio et al. die Werte für das 95. Perzentil etwas höher liegen als bei Allard et al., kann man davon ausgehen, dass auch hier der Anteil an Betroffenen etwas niedriger läge. Störungen der Glukosetoleranz zeigten sich insgesamt eher selten. Das Screening der Nüchternglukose ergab bei Jungen häufiger als bei Mädchen auffällige Werte. Im OGTT waren dann aber beide Geschlechter prozentual von allen gescreenten Kindern und 
Jugendlichen gleich häufig betroffen. Mit dem Alter stieg der Anteil an auffälligen GlukoseScreenings. Die Werte der betroffenen Kinder zwischen null und neun Jahren lagen alle im Bereich von 100-105 mg/dl und wären somit nach anderen Definitionen [Ford und Li 2008] nicht einmal pathologisch. In US-amerikanischen Kollektiven mit Jugendlichen > 85. BMIPerzentil kommt eine gestörte Glukosetoleranz mit 49\% deutlich häufiger vor als in unserem Kollektiv [Cook et al. 2008]. Manifester Diabetes Mellitus Typ 2 (T2DM) zeigte sich nur in einem von 376 Fällen. Das deckt sich mit Literatur aus den USA, wo trotz einer hohen Adipositas-Rate von insgesamt 17\% (>95. BMI-Perzentil) bei Kindern und Jugendlichen die Prävalenz von T2DM unter 0,5\% liegt [Cook et al. 2003]. Bei Jugendlichen unter 20 Jahren mit T2DM fanden sich in über $92 \%$ der Fälle zwei oder mehr andere metabolische Auffälligkeiten (arterielle Hypertonie, vermindertes HDL, erhöhte Triglyceride oder abdominelle Adipositas) [Cook et al. 2008].

Das war auch bei unserem Patienten der Fall, welcher, neben T2DM, zudem eine abdominelle Adipositas, vermindertes HDL und eine arterielle Hypertonie aufwies.

\subsection{Steatosis hepatis}

Für Steatosis hepatis fanden sich Abhängigkeiten von Geschlecht, Alter und BMI-SDS. Deutlich am häufigsten betroffen waren die älteren Jungen mit höherem BMI-SDS, wobei das Alter einen stärkeren Einfluss hatte. In einer Untersuchung mit 13.581 Patienten fanden Wiegand et al. (2010) mit 11,5\% einen ähnlich hohen Gesamtanteil wie in unserer Studie $(10,57 \%)$ bei gleichem Cut-Off-Wert von $50 \mathrm{U} / \mathrm{l}$ für AST und ALT. Bei diesem, relativ hoch gewählten, Wert muss man allerdings von einer größeren Zahl falsch-negativer Ergebnisse ausgehen. Eine definitive Diagnose kann nur bioptisch gestellt werden. In Obduktionsstudien waren $38 \%$ der adipösen Kindern und Jugendlichen betroffen [Schwimmer et al. 2009]. Unter Verwendung der allgemeingültigen alterspezifischen Normwerte ergaben sich Anteile von 22-53\% bei übergewichtigen Kindern [Keller KM 2004]. Einige Autoren, wie Di Bonito et al. (2009), schlugen die Festlegung alters- und geschlechtsspezifische Cut-Off-Werte für die Transaminasen vor.

\subsection{Fettstoffwechselstörungen}

Eine Erhöhung des LDL-Cholesterin kommt im untersuchten Kollektiv mit einer Inzidenz zwischen $30,69 \%$ und $49,18 \%$ häufig vor, ist jedoch meist moderat ausgeprägt. Es fand sich erwartungsgemäß kein Zusammenhang mit Alter oder BMI-SDS. Ein vermindertes HDL wurde mit zunehmendem Alter relevanter, zeigte aber hier keinen Zusammenhang mit dem Ausmaß der Adipositas. Eine Erhöhung der Triglyceride ist bei beiden Geschlechtern mit 
insgesamt 46,09\% die häufigste Co-Morbidität in unserem Kollektiv. Beide Geschlechter sind etwa gleich stark betroffen. Es konnte kein relevanter Zusammenhang mit dem Alter oder BMI-SDS gezeigt werden.

\subsection{Arterielle Hypertonie}

Essenzielle Hypertonie stellt eher eine seltene Diagnose im Kindes- und Jugendalter dar. Bei der Auswertung der 24-Stunden-Blutdurckmessungen anhand der Perzentile nach Geschlecht und Körpergröße erfüllten 9,62\% die Kriterien für eine arterielle Hypertonie oder schwere arterielle Hypertonie. Bezogen auf alle Patienten, deren Blutdruck gemessen wurde, entspricht das einem Anteil von 1,18\%. Bei einer Auswertung entsprechend der Perzentile nach Geschlecht und Alter läge der Anteil Betroffener mit 2,13\% etwas höher, was in absoluten Zahlen vier zusätzlich betroffenen Jungen entspricht. Erklären ließe sich das mit der höheren durchschnittlichen Körpergröße im Bezug auf das Alter bei Adipösen. Der durchschnittliche Größen-SDS der betroffenen vier Jungen lag bei 1,12 $\pm 0,75$. Durch die insgesamt kleine Anzahl Betroffener im Kollektiv ist keine valide Aussage über eine Abhängigkeit von Alter oder BMI-SDS zu treffen. In der Literatur schwankt die Prävalenz bei US-amerikanischen Kindern und Jugendlichen je nach Definition zwischen 4,3-9,9\% bei Übergewichtigen und 13,0-23,6\% bei Adipösen [Cook et al. 2008].

\subsection{Zusammenfassung zum metabolischen Syndrom}

Die Definition des metabolischen Syndroms ist immer noch umstritten [Saland 2007]. Bei vielen Autoren herrscht zwar Einigkeit über die Komponenten des metabolischen Syndroms, jedoch unterscheiden sich die einzelnen Cut-Off-Werte zum Teil deutlich [Ford und Li 2008]. Nach Janner et al. (2006) werden zu den Komponenten des metabolischen Syndroms, entsprechend der Leitlinien der International Diabetes Foundation für Erwachsene [IDF 2006], gestörte Glukosetoleranz (pathologischer HOMA-IR, pathologische Nüchtern-Glukose oder pathologischer OGTT), arterielle Hypertonie, vermindertes HDL, erhöhte Triglyceride und Adipositas gezählt. Laut AGA sollten in diesem Zusammenhang auch Transaminasen und Harnsäure bestimmt werden [AGA 2006]. Neben der körperlichen Konstitution spielt die Genetik eine zentrale Rolle bei der Entstehung des metabolischen Syndroms, es konnten sogar Regionen im Genom identifiziert werden, die direkte Einfluss auf den Glukosestoffwechsel des Menschen haben [Saland 2007]. Über 60\% der Kinder und Jugendlichen des untersuchten Kollektivs waren von mindestens einer der oben genannten Störungen betroffen. Jungen sind häufiger (37,6\%) von zwei oder mehr Störungen betroffen als Mädchen (26,60\%). Das Alter scheint hierbei einen stärkeren Einfluss zu nehmen als 
das Ausmaß der Adipositas. Bei Mädchen konnte gar kein Zusammenhang zwischen BMISDS und Anzahl der Co-Morbiditäten hergestellt werden.

Gleichzeitig drei oder mehr Kriterien nach Janner et al. (2006), und somit die Definition für das metabolische Syndrom, erfüllten insgesamt 15,07\% der Mädchen und 15,70\% der Jungen.

In der NHANES III Studie wurden 2340 US-amerikanische Jugendliche zwischen zwölf und 19 Jahren mit den gleichen Parametern auf das metabolische Syndrom untersucht. Es ergab sich bei den Adipösen (hier definiert als $>95$. Perzentil) eine Prävalenz zwischen $12,4 \%$ und $44,2 \%$ je nach Definition [Cook et al. 2008]. In der gleichen Altersgruppe (>12 Jahre) lag der Anteil der Betroffenen in unserem Kollektiv mit 19,02\% im unteren Bereich dieser Spanne.

Das metabolische Syndrom geht auch noch mit anderen Auffälligkeiten einher, die zum Beispiel die Entzündungs-, Hämolyse- und Fibrinolyseparamter betreffen. Diese sind zurzeit Gegenstand intensiver Forschung [Ford und Li 2008]. Die Herausforderung an die Experten ist, den gemeinsamen Nenner dieser Störung zu finden. Hierfür werden abdominelle Adipositas und Insulinresistenz diskutiert: 85\% der Adipösen aller Altersgruppen sind in gewissem Maße von Insulinresistenz betroffen [IDF 2006]. Der HOMA-IR hat sich hierbei in mehreren Studien als verlässliches Maß für Insulinresistenz bewährt [Caceres et al. 2008, d'Annunzio et al. 2008]. In unserem Kollektiv weisen 98,04\% der Kinder mit auffälligem HOMA-IR gleichzeitig einen BMI-SDS >2 und 49,02\% zusätzlich eine erhöhte Triglyceridkonzentration im Serum auf. Bei 21,57\% zeigte sich bei auffälligem HOMA-IR neben der Adipositas eine gestörte Glukosetoleranz und 9,80\% sind sogar von einem BMISDS > 2, erhöhten TG und gestörter Glukosetoleranz betroffen. Ein Problem bei der Erfassung von Stammfettsucht bei Jungendlichen ist, dass es bisher zwar den Versuch [Ford und Li 2008], aber kein allgemeingültiges standardisiertes Verfahren und keine Referenzwerte gibt [Cook et al. 2008]. Dennoch gibt es Hinweise, dass Stammfettsucht bei Kindern und Jungendlichen in den letzten Jahren schneller zunimmt als die Adipositas an sich [Cook et al. 2008].

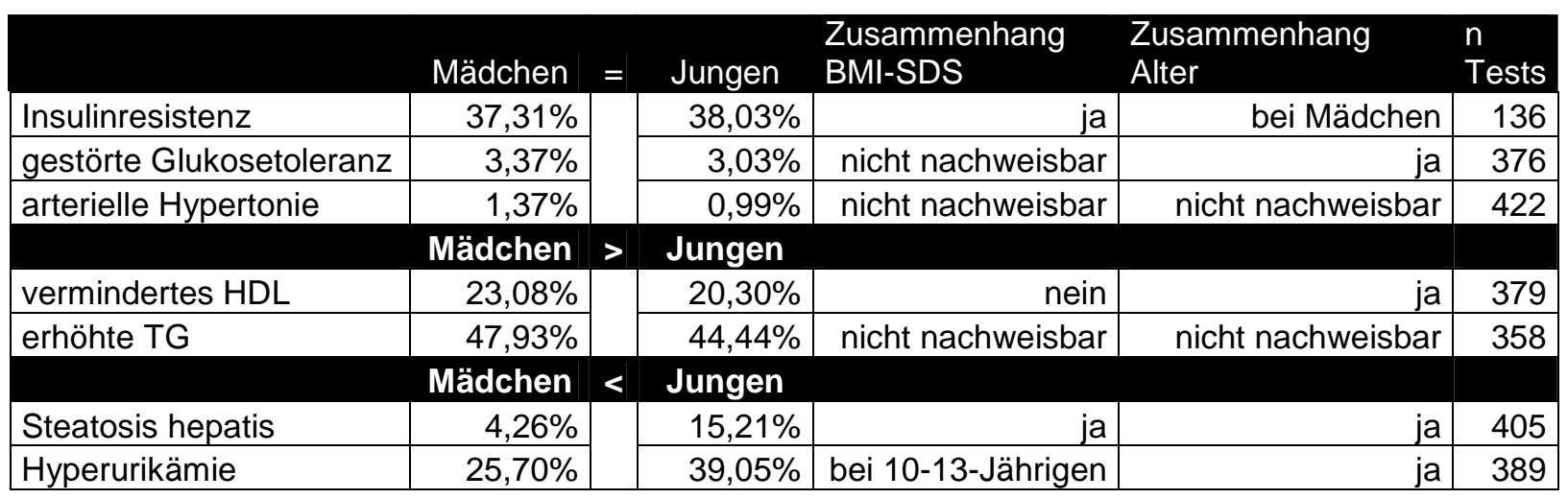

Tabelle 28: Übersicht zur Inzidenz von Begleiterkrankungen im Kollektiv 


\subsubsection{Hyperurikämie}

Bei der Hyperurikämie handelt es sich um eine häufige Co-Morbidiät. Betroffen sind vor allem die ältern Jungen. Bei den über 14-Jährigen waren es über $80 \%$. Insgesamt ist die Harnsäure bei $32,13 \%$ der Patienten erhöht. In der Literatur wird die Prävalenz der Hyperurikämie bei übergewichtigen Kindern und Jugendlichen mit 20\% und somit niedriger angegeben [Reinehr 2005]. Nach Alper et al. (2005) sollte auch im Kindes- und Jugendalter eine Normalisierung der Harnsäure zur Prävention einer arteriellen Hypertonie angestrebt werden. Die Reduktion der Harnsäure um $1 \mathrm{mg} / \mathrm{dl}$ führt zu einer Abnahme des systolischen Blutdrucks von $3 \mathrm{mmHg}$ [Franco et al. 2006].

\subsubsection{Weitere kardiovaskuläre Risikofaktoren}

\subsection{Lipoprotein(a)}

Lp(a) wurde als unabhängiger kardivaskulärer Risikofaktor diagnostiziert. Die Bedeutung des Ausmaßes der Erhöhung ist weiterhin unklar [Kraft und Utermann 2007]. Fest steht, dass die Höhe der Werte zwischen den Individuen stark schwankt (0,5-400 mg/dl), in ein und derselben Person jedoch konstant ist [Kraft und Utermann 2007], was auf den Polymorphismus des codierenden Apo(a)-Gens zurückgeführt wird [Dieplinger und Kronenberg 1999]. Über 50\% der Bevölkerung haben Werte unter 10 mg/dl [Kraft und Utermann 2007]. In unserem Kollektiv lagen 60,48\% der Kinder unterhalb des von der AGA festgesetzten Grenzwertes von 30mg/dl.

\subsection{Hyperhomozytseinämie}

Das Homozystein war bei keinem der Kinder erhöht. Das zeigt, dass sehr wahrscheinlich bei keinem der Patienten eine genetische Disposition oder, trotz mutmaßlich suboptimaler Zusammensetzung der Ernährung, ein Folsäure-, Vitamin B6- oder Vitamin B12-Mangel besteht.

\subsubsection{3. Übersicht über die zusätzlichen kardiovaskulären Risikofaktoren}

Zusätzliche kardiovaskuläre Risikofaktoren, die hier analysiert, aber nicht zum metabolischen Syndrom gezählt werden, sind Homozystein, LDL-Cholesterin und Lp(a). Homozystein war bei allen untersuchen Patienten im Normbereich, jedoch fand sich bei über der Hälfte der Jungen und sogar über zwei Drittel der Mädchen eine Erhöhung von mindestens einem 
zusätzlichen Faktor. Tritt ein erhöhtes LDL kombiniert mit einem erhöhten Lp(a) auf, steigt auch das Risiko für Apoplex und Myocardinfarkt dieser Patienten um ein Vielfaches an [Kraft und Utermann 2007]. Das trifft in unserem Kollektiv auf 18,84\% der Jungen und $15,00 \%$ der Mädchen zu, die daher besonderer präventiver Maßnahmen bedürfen.

\subsubsection{Leptin}

Bei keinem der Kinder oder Jugendlichen fand sich ein Hinweis auf einen genetisch determinierten Leptinmangel. Die lineare Regression von Leptin-Spiegel und BMI-SDS ergibt bei den Jungen die erwartete positive Korrelation. Das Ergebnis für die Mädchen ist jedoch nicht eindeutig. Eine starke und signifikante Korrelation mit $r=0,53$ und $p=0,019$ fand sich nur bei den ältesten Mädchen zwischen 14-18 Jahren. Der Leptinwert ist direkt proportional zum Körperfettanteil [Kelesidis et al. 2010], wobei der Grad der Abhängigkeit im Verlauf der Geschlechtsentwicklung physiologischen Schwankungen unterliegt. Deshalb sollten bei Kindern und Jungendlichen Perzentile nach Tanner-Stadium für die Bestimmung der Grenzwerte verwendet werden [Blum et al. 1997]. Außerdem unterliegt der LeptinSpiegel einer zirkadianen Rhythmik [Kelesidis et al. 2010], weshalb die Blutentnahme zu einer standardisierten Tageszeit erfolgen sollte. Diese Tatsachen sind mögliche Erklärungen für die nicht eindeutigen Ergebnisse bei den Mädchen.

\subsection{Verlauf vor der Intervention}

\subsubsection{Analyse der Daten aus den Vorsorgeuntersuchungen}

Sowohl bei den Jungen als auch bei den Mädchen sind die betroffenen Kinder und Jugendlichen schon in sehr jungem Alter signifikant dicker als der Durchschnitt der deutschen Kinder. Besonders deutlich ist der Unterschied bei beiden Geschlechtern bei der U8 und U9 mit vier (43.-48. Lebensmonat) bzw. sechs Jahren (60.-64. Lebensmonat), so dass es hier möglicherweise für präventive Maßnahmen bereits zu spät ist. Es sollte daher zum Zeitpunkt der U6 und U7 (10.-12. Lebensmonat bzw. 21.-24. Lebensmonat) auf Gewichtsverlauf und Risikofaktoren bei den Kindern geachtet werden, da in diesem Alter der BMI-SDS im Vergleich zu gesunden Kindern wieder stärker zu steigen beginnt.

Eine niederschwellige Intervention, wie das Führen eines Ernährungs- und Bewegungstagebuches mit anschließender Beratung durch den Kinderarzt sowie die Vermittlung von Sportangeboten, wird von den Patienten und ihren Familien in der Regel gut akzeptiert. Es konnte gezeigt werden, dass diese Maßnahmen das weitere Ansteigen des BMI-SDS verhindern können [Keller A et al. 2009]. Ein Problem stellt hier auch die Tatsache 
dar, dass die U7 und U8 zeitlich weit auseinander liegen und so gerade sozial Schwache, die nachweislich häufiger von Adipositas betroffen sind, der externen Kontrolle durch den Kinderarzt entgehen können. Am 01.07.2008 wurde glücklicherweise die U7a (34. bis 36. Lebensmonat) eingeführt, die dieses lange Intervall zwischen U7 und U8 überbrücken soll [Ärztezeitung 2008].

\subsubsection{Regression des Gewichtsverlaufes vor der Intervention}

Adipositas hat ihren Ursprung bereits im frühen Lebensalter [Adair 2008]. Das zeigt sich auch in unserer polynomischen Regression, die uns einen Anhalt für den mittleren Verlauf des BMI der Jungen und Mädchen geben soll. Das Polynom schneidet das 97. Perzentil schon im Kleinkindalter (Mädchen: 2 Jahre, Jungen: 2-3 Jahre) und verläuft ab dem Adipositas-Rebound zu jeder Zeit steiler als die Perzentile nach Kromeyer-Hauschild et al. bis es schließlich bei beiden Geschlechter etwa um das 14. Lebensjahr das 99,5. Perzentil zur extremen Adipositas schneidet.

Ein früher Adipositas-Rebound (Mädchen <5,5 Jahre, Jungen <5 Jahre) ist ein Risikofaktor für eine frühe Entstehung von Adipositas [Williams und Goulding 2008]. Rechnerisch erhält man für die Wendepunkte der Funktion mehrere Ergebnisse, von denen visuell der jeweils erste Wert am ehesten dem AR entspricht. Dieser liegt bei den Jungen bei 2,8 und bei den Mädchen bei 3,6 Jahren, was nach der aktuellen Literatur einem sehr frühen $A R$ entsprechen würde.

Der Verlauf des BMI in Abhängigkeit vom Alter folgt keiner mathematischen Gesetzmäßigkeit, so ist die polynomische Regression hier die bestmögliche, aber keine perfekte Schätzung des mittleren BMI-Verlaufs. Daher handelt es sich auch bei den errechneten Wendepunkten für den Adipositas-Rebound lediglich um einen Anhaltspunkt.

\subsubsection{Zusammenhang mit Geburtsgewicht und Geburtswoche}

Ein besonders hohes oder besonders niedriges Geburtsgewicht [Röbl et al. 2006] und Frühgeburtlichkeit [Kries et al. 2002] gelten als Risikofaktoren für die Entstehung einer frühen Adipositas. In der KiGGS-Studie konnte ein Geburtsgewicht über 4000g mit einer OddsRatio von 1,73 als unabhängiger Risikofaktor für spätere Adipositas identifiziert werden. Von 13450 untersuchten Kindern waren insgesamt 2601 oberhalb des 85. Perzentils nach Cole und somit übergewichtig oder adipös. Bei 15,84\% dieser Kinder lag ein Geburtsgewicht von über $4000 \mathrm{~g}$ vor, während der Anteil hypertropher Kinder am Gesamtkollektiv 10,79\% betrug [Kleiser et al. 2009]. Demgegenüber ist auch der Anteil der bei Geburt hypertroph geborenen Kinder in unserer Untersuchung mit insgesamt 19,56\% erhöht, wobei die Jungen 
$(23,71 \%)$ stärker betroffen sind als die Mädchen (14,79\%), die ehemals hypertrophen Mädchen haben jedoch einen höheren mittleren BMI-SDS als die ehemals eutrophen Mädchen. Auch der Anteil Fühgeborener zeigt sich im gesamtdeutschen Vergleich mit insgesamt 11,31\% erhöht. Die Frühgeborenenrate in Deutschland betrug 2001 6\% [Hahne 2001]. Die Mädchen betraf dies mit 13,42\% häufiger als die Jungen. Die ehemaligen Frühgeborenen des Kollektivs sind aber nicht schwerer betroffen als die termingerecht geborenen Kinder.

\subsection{Therapieerfolg}

74,73\% der Patienten, die nach einem Jahr noch in Therapie waren, konnten ihren BMI-SDS in dieser Zeit reduzieren. Als Therapieerfolg ist die Reduktion des BMI-SDS um 0,5 nach einem Jahr definiert, da es ab dieser BMI-SDS-Reduktion zu einer nachweisbaren Reduktion des kardiovaskulären Risikos kommt [Reinehr et al. 2009]. Diese Definition trifft bei uns nur bei $12,09 \%$ der Kinder zu, deren biometrische Daten nach einem Jahr vorlagen. Bezogen auf alle Kinder, die sich in der Adipositasambulanz vorstellten, entspricht das einem Anteil von 3,25\%, wobei diese Daten nur von einem Viertel der Kinder erhoben werden konnten. Die Problematik dieser hohen Abbrecherrate (hier 67,32\% nach sechs, 80,30\% nach zwölf Monaten) zeigte sich auch in anderen Studien [Reinehr et al. 2009] und stellt ein Problem für die Zuverlässigkeit der Datenauswertung dar, da als möglicher Grund für den Therapieabbruch einerseits ein ausreichender Erfolg, andererseits aber auch die Resignation in Frage kommen kann. So wäre es möglich, dass die wahre Erfolgsrate höher liegt und nur nicht erfasst werden konnte. Auch die durchschnittliche BMI-SDS-Reduktion muss mit 0,15 $\pm 0,26$ nach sechs und 0,17 $\pm 0,30$ nach zwölf Monaten als gering bewertet werden.

In einer Untersuchung von Reinehr et al. (2009), die an 21.784 Kinder aus ambulanten Zentren in Deutschland, Österreich und der Schweiz durchgeführt wurde, zeigte sich bei einer Auswertung der Daten der fünf erfolgreichsten Zentren (518 Kinder) eine Reduktion des BMI-SDS nach sechs Monaten bei $83 \%$ und nach zwölf Monaten Intervention bei $67 \%$ der Kinder eine Reduktion des BMI-SDS. Grundsätzlich positiv zu bewerten ist die Tatsache, dass von den Kindern, die nach einem Jahr noch in Therapie waren, über die Hälfte $(58,18 \%)$ sowohl im ersten und als auch im zweiten Therapiehalbjahr ihren BMI-SDS verringern konnten.

Ein Drittel der Kinder konnte den BMI-SDS initial reduzieren, verlor aber im zweiten Therapiehalbjahr die Motivation und rund 16\% haben nach einem erfolglosen ersten Therapiehalbjahr ihren BMI-SDS im zweiten Halbjahr doch noch vermindern können. Gerade um diese verschiedenen Verläufe zu erkennen, ist es wichtig, dass die Therapiedauer nicht zu kurz gewählt wird. Nur knapp 10\% hatten nach sechs und zwölf 
Monaten trotz Therapie jeweils weiter zugenommen. Diese Patienten waren im Durchschnitt älter als alle anderen Patienten des Kollektivs. Je jünger die Kinder sind, desto besser ist der Therapieerfolg sowohl nach einem halben als auch nach einem Jahr. In anderen Studien konnte diese Tatsache auch für eine Therapiedauer von zwei Jahren gezeigt werden. Die Kinder unter zwölf Jahren hatten hier, im Gegensatz zu den Jugendlichen über zwölf Jahren, eine Odds-Ratio von 4,1 ihren BMI-SDS innerhalb von zwei Jahren Therapie zu reduzieren [Reinehr et al. 2009]. Betrachtet man Jungen und Mädchen getrennt, so ist die Gewichtsreduktion nach sechs Monaten Interventionsdauer bei beiden Geschlechtern etwa gleich. Nach einem Jahr beweisen die Jungen allerdings ein signifikant besseres Durchhaltevermögen. Bei Reinehr et al. zeigte sich nach zwei Jahren kein Erfolgsunterschied der Geschlechter. Am besten haben in unserem Kollektiv die Jungen unter zehn Jahren, am schlechtesten die Mädchen von 14 bis 18 Jahren abgenommen.

\subsubsection{Therapiegruppen}

Die größte Gruppe bilden bei sechsmonatiger Therapiedauer die Kinder in Einzelberatung. Sie haben auch nach sechs und zwölf Monaten die beste BMI-SDS-Reduktion. Diese Patienten nahmen individuell nach Bedarf nur psychologische oder Ernährungsberatung in Anspruch, wobei der größte Anteil mit rund 80\% nur Ernährungsberatung erhielt.

Kinder und Jugendliche, die von einem multiprofessionellen Team betreut werden, bildeten nach einjähriger Therapiedauer die größte Gruppe. Sie sind am schwersten von Adipositas betroffen und nehmen am schlechtesten ab. Alle diese Patienten nehmen psychologische Hilfe in Anspruch.

Oft ist es schwer zu unterscheiden, ob eine psychische Co-Morbidität, wie zum Beispiel eine Depression oder Angststörung, Folge oder Ursache der Adipositas darstellt [Warschburger 2006]. Hier kann sie aber als möglicher Prädiktor für schlechten Therapieerfolg diskutiert werden. Die Schulung nach den Leitlinien der AGA umfasst eine definierte Anzahl an Ernährungsberatung, Verhaltenstherapie und Bewegungstherapie für Eltern und Kinder in der Gruppe. Diese Patienten haben die aufwändigste Therapie hinter sich gebracht, aber trotzdem nicht den besten Erfolg. Nach sechs Monaten haben sie den mittleren BMI-SDS sogar am schlechtesten reduziert. Das wirft die Frage auf, ob Adipositastherapie nach standardisierten, immer gleichen Richtlinien erfolgen kann oder für jedes Kind bzw. jeden Jugendlichen andere Anforderungen erfültt werden müssen. Reinehr et al. (2009) konnten zeigen, dass zwar die Therapiedichte, nicht aber die Therapiedauer einen günstigen Einfluss auf das Outcome haben. 


\subsubsection{Psychosoziale Faktoren}

\subsubsection{Migrationshintergrund}

Anfänglich lag der Antel von Kindern und Jugendlichen mit Migrationshintergrund bei 15,70\% und nahm nach sechs Monaten auf fast $20 \%$ zu. Sie waren öfter ein halbes Jahr in Therapie als Patienten ohne Migrationshintergrund. Nach einem Jahr war der Migrantenanteil wieder genauso hoch wie zu Therapiebeginn. Nach einem Jahr haben also Kinder und Jugendliche mit und ohne Migrationshintergrund im selben Maße die Therapie abgebrochen. Die Migrantenkinder, die nach einem Jahr noch in Behandlung waren, konnten ihren BMI-SDS etwas besser reduzieren als die deutschstämmigen Kinder.

\subsubsection{Ausbildung und Berufstätigkeit der Eltern}

In unserer Analyse konnte kein direkter Einfluss des Bildungsstandes der Eltern auf den BMI-SDS der Kinder nachgewiesen werden. Die Aussagekraft der Daten ist allerdings durch die kleine Stichprobengröße gering. Kinder von Müttern mit Abitur waren am seltensten nach sechs oder zwölf Monaten noch in Therapie, was daran liegen könnte, dass sie die Therapieinhalte am schnellsten begreifen und eigenständig zu Hause umsetzen können. Kinder und Jugendliche, deren Eltern keinen, einen Sonder- oder Hauptschulabschluss haben, sind am häufigsten nach einem halben und einem Jahr noch in Therapie und brauchen vielleicht etwas mehr professionelle Unterstützung.

Söhne und Töchter von berufstätigen Müttern haben nach sechs und zwölf Monaten Intervention ein besseres Outcome als die Kinder von Hausfrauen. Zwischen Berufstätigkeit der Väter und Therapieerfolg der Patienten konnte kein Zusammenhang hergestellt werden.

\subsubsection{Weiterführende Schulbildung der Kinder und Jugendlichen}

Die eigene Bildung der Kinder und Jugendlichen scheint einen größeren Einfluss auf den Therapieerfolg zu nehmen als die Bildung der Eltern. Patienten, die ein Gymnasium besuchen, haben nach einem halben und einem Jahr Therapie das deutlich beste Ergebnis. Die fehlende Signifikanz kann auch hier eventuell durch die geringe Stichprobengröße erklärt werden. Vergleicht man den Therapieerfolg der Hauptschüler mit dem der Kinder und Jugendlichen, die andere weiterführende Schulen besuchen, sieht man, dass der Therapieerfolg der Hauptschüler deutlich schlechter ist. 


\section{Zusammenfassung}

In dieser Studie wurden die Daten von 462 Kindern und Jugendlichen (219 Mädchen, 243 Jungen) untersucht, die sich zwischen dem 01.03.2005 und 29.02.2008 erstmals in der Adipositas-Sprechstunde des Sozialpädiatrischen Zentrums (SPZ) Göttingen vorstellten. Das Durchschnittsalter lag bei 10,53 Jahren bei den Mädchen und 11,07 Jahren bei den Jungen (Spannbreite 3 Monate bis 18 Jahre). Bei der Erstvorstellung war der größte Teil der Patienten (40,7\%) zwischen 11 und 14 Jahren alt. In dieser Altersgruppe zeigte sich jedoch gleichzeitig die geringste Ausprägung der Adipositas. Die Mädchen waren bei Erstvorstellung signifikant in stärkerem Maße von Adipositas betroffen als die Jungen: der größte Anteil $(53,5 \%)$ der Jungen war bei Erstvorstellung adipös, der größte Teil der Mädchen (48,9\%) extrem adipös.

Als Risikofaktoren wurden mit signifikantem Unterschied im BMI-SDS die Parameter Einzelkind-Status, Kinder und Jugendliche mit mehr als zwei Geschwistern, Adipositas der Eltern, Mütter ohne Abitur sowie Kinder und Jugendliche, die eine Hauptschule besuchen, identifiziert. Ein weiterer Risikofaktor für eine Adipositas im Kindes- und Jugendalter ist ein frühzeitiger Adipositas-Rebound. Bei der Analyse der Daten aus den gelben Kindervorsorgeheften zeigte sich, dass die Patienten schon früh aus ihren BMI-Perzentilen ausbrechen, der Adipositas-Rebound deutlich früher stattfindet und die Kurven insgesamt steiler verlaufen als im deutschen Vergleichskollektiv. Kein Unterschied im BMI-SDS bei Erstvorstellung konnte für eine unterschiedliche familiäre Situation (allein erziehend/ nicht allein erziehend), Migrationshintergrund oder Berufstätigkeit der Eltern festgestellt werden.

Folgeerkrankungen der Adipositas treten bereits im Kindes- und Jugendalter auf: bei $60,73 \%$ der Mädchen und 63,22\% der Jungen lag bei Erstvorstellung schon mindestens eine der untersuchten metabolischen Störungen wie Insulinresistenz, pathologische Glukosetoleranz, Dyslipoproteinämie, Hyperurikämie, Steatosis hepatis oder arterielle Hypertonie vor. Die Inzidenz von Begleiterkrankungen nahm mit dem Alter und BMI-SDS zu. Mädchen waren häufiger als Jungen von einer Dyslipoproteinämie im Sinne von Hypertriglyceridämie und vermindertem HDL-Cholestierin betroffen. Bei den Jungen zeigte sich dagegen häufiger eine Hyperurikämie und eine Steatosis hepatis. Insulinresistenz gestörte Glukosetoleranz oder arterielle Hypertonie wurde bei beiden Geschlechtern etwa gleich häufig festgestellt. Die Definition des metabolischen Syndroms bei Kindern und Jugendlichen ist umstritten. Die in dieser Studie angewendeten Kriterien nach Janner et al. (2006) erfüllen 15,07\% der Mädchen und 15,70\% der Jungen des untersuchten Kollektivs. 
Nicht alle Kinder und Jugendlichen, die sich im Sozialpädiatrischen Zentrum vorstellten, ließen sich auch auf eine therapeutische Intervention ein. So wurde die Evaluation des Therapieerfolges durch eine hohe Abbrecherrate (67,32\% nach sechs, 80,30\% nach zwölf Monaten Interventionsdauer) erschwert. $74,73 \%$ der Kinder und Jugendlichen, die nach einem Jahr noch in Therapie waren, konnten ihren BMI-SDS in dieser Zeit im Mittel um 0,28 $\pm 0,23$ reduzieren. $12,09 \%$ erfüllten die Kriterien für eine sehr erfolgreiche Therapie (BMISDS-Reduktion $>0,5$ ), die eine nachweisliche Senkung des kardiovaskulären Risikos mit sich bringt. Kleinere Kinder bis neun Jahre und Jungen zeigen nach einem Jahr die besten Ergebnisse. Einen besonders hohen Therapieaufwand benötigen Mädchen, ältere Jungendliche ab 14 Jahren, Hauptschüler, Patienten mit psychischer Co-Morbidität oder mit Müttern mit niedrigem Bildungsstand. Der Therapieerfolg war weitgehend unabhängig von Migrationshintergrund und Bildungsstand des Vaters.

Die durchgeführte Studie bestätigt, dass die Therapie der Adipositas im Kindes- und Jungendalter schwierig ist und die verantwortlichen Ärzte und Therapeuten vor eine Herausforderung stellt, insbesondere, wenn familiäre und soziale Faktoren eine Adipositas begünstigen und den Therapieerfolg zusätzlich bremsen. Adipositas hat ihre Wurzeln bereits in der frühen Kindheit, so dass die Anzeichen, wie zum Beispiel ein früher AdipositasRebound, nicht übersehen werden dürfen. Je jünger der Patient ist, desto Erfolg versprechender ist ein Therapieversuch. Begonnen werden sollte mit niederschwelligen Interventionen, die zunächst mit Hilfe des Kinderarztes in den Alltag integriert werden können. Stellen sich hierdurch keine Änderungen der Lebensgewohnheiten ein, so dass es zu einer weiteren Gewichtszunahme kommt, sollte die Überweisung in eine spezialisierte Einrichtung erfolgen. Auf Kinder und Jugendliche, die in einem adipogenen Umfeld aufwachsen, muss hierbei ein besonderes Augenmerk gerichtet und ein präventiver Ansatz forciert werden. Sollten bereits Übergewicht oder Adipositas vorliegen, so ist eine Untersuchung der möglichen Folgeerkrankungen unbedingt notwendig, da diese auch schon im Kindes- und Jugendalter auftreten. 


\section{Literaturverzeichnis}

Adair LS (2008): Child and adolescent obesity: epidemiology and devenlpmental perspectives. Physiol Behav, 94:8-16

AGA (Arbeitsgemeinschaft Adipositas im Kindes- und Jugendalter) (2006): Leitlinien. http://www.a-g-a.de/Leitlinie.pdf (Letzter Zugriff 20.01.2008)

Allard P, Delvin EE, Paradis G, Hanley JA, O'Loughlin J, Lavalée C, Levy E, Lambert M (2003): Distribution os Fasting Plasma Insulin, Free Fatty Acids, and Glukose Concentrations and of the Homeostasis Model Assessment of Insulin Resistance in a Representative

Sample of Quebec Children and Adolescents. Clin Chem, 49:644-649

Alper AB Jr, Chen W, Yau L, Srinivasan SR, Berenson GS, Hamm L (2005): Childhood Uric Acid Predicts Adult Blood Pressure. The Bogalusa Heart Study. Hypertension, 45:34-38

D’Annunzio G, Vanelli M, Pistorio A, Minuto N, Bergamino L, lafusco D, Lorini R, Diabetes Study Group of the Italian Society for Pediatric Endocrinology and Diabetes (ISPED) (2008): Insulin resistance and secretion indexes in healthy Italian children and Adolescents: a muticentre study. Acta Biomed, $\underline{80}: 21-28$

Ärztezeitung (2008): Kleiner Punktevorteil für die neue U7a.

http://www.aerztezeitung.de/praxis_wirtschaft/aerztliche_verguetung/article/502472/kleinerpunktevorteil-neue-u7a.html (Letzter Zugriff 27.03.2011)

Blum WF, Kiess W, Rascher W: Leptin The Voice of Adipose Tissue. Johann Ambrosius Barth Verlag Heidelberg - Leipzig 1997

Bös K (2001): Bündnis "gesunde Kinder": unsere Kinder brauchen mehr Bewegung, Spiel und Sport. Vortrag auf dem Stuttgarter Sportkongress am 09.11.2001. http://www.sport.unikarlsruhe.de/ifss/seite_837.html (Letzter Zugriff 20.04.2008)

Britz B, Siegfried W, Ziegler A, Lamertz C, Herpertz-Dahlmann BM, Remschmidt H, Wittchen $\mathrm{H}-\mathrm{U}$, Hebebrand J (2000): Rates of psychiatric disorders in a clinical study group of adolescents with extreme obesity and in obese adolescents ascertained via a population based study. Int J Obes, 24:1707-1714

Bühling KJ, Friedmann W: Intensivkurs Gynäkologie und Geburtshilfe, 1. Auflage; Urban \& Fischer Verlag, München - Jena 2004

Caceres M, Teran CG, Rodriguez S, Medina M (2008): Prevalence of insulin resistance and its assiciation with metabolic syndrome criteria among Bolivian children and adolescents with obesity. BMC Pediatr, $\underline{8}: 31-36$

Ceders-Sinai (2010): Obesity and Insulin Resistance. http://www.cedarssinai.edu/Patients/Programs-and-Services/Androgen-Related-Research-and-DiscoveryCenter/Symptoms-and-Diagnosis/Obesity-and-Insulin-Resistance.aspx (Letzter Zugriff 28.09.2010)

Chen C, Visootsak J, Dills S, Graham JM Jr. (2007): Prader-Willi Syndrome: An Update and Review for the Primary Pediatrician. Clin Pediatr, $\underline{46}: 580-589$

Cole TJ, Freeman JV, Preece MA (1990): Body mass index reference curves for the UK, 1990. Arch Dis Child, $\underline{73}: 25-29$ 
Cole TJ, Bellizzi MC, Flegal KM, Diezt WII (2000): Establishing a standard defintion for child overweight and obesity worldwide: international survey. BMJ, 320:1240-1243

Cook S, Weitzman M, Auinger P, Nguyen M, Dietz WH (2003): Prevalence of the Metabolic Syndrome Phenotype in Adolescents, Findings form the Third National Health and Nutrition Examination Survey, 1988-1994. Arch Pediatr Adolesc Med 157:821-827

Cook S, Auinger P, Li C, Ford ES (2008): Metabolic Syndrome Rates in United States Adolescents, from the National Health and Nutrition Survey, 1999-2002. J Pediatr, 152(2): $165-170$

Davis M, Gance-Cleveland B, Hassink S, Johnson R, Paradis G, Resnicow K (2007): Recommendations for Prevention of Childhood Obesity. Pediatrics, 120:229-253

Di Bonito P, Sanguigno E, Di Fraia T, Forziato C, Boccia G, Saitta F, lardino MR, Capaldo B (2009): Association of elevated serum alanine aminotransferase with metabolic factors in obese children: sex-related analysis. Metabolism, 58(3):368-372

Dieplinger H, Kronenberg F (1999): Genetics and metabolism of lipoprotein(a) and their clinical implications (Part1). Wien Klin Wochenschr 15(111):5-20

Dixon HG, Scully ML, Wakefield MA, White VM, Crawford DA (2007): The effects of television advertisementsfor junk food versus nutritious food on children's food attitudes and preferences. Soc Sci Med, $\underline{65}: 1311-1323$

Dordel S: Haltungs- und Bewegungsapparat; in: Bewegungsmangel und Fehlernährung bei Kindern und Jugendlichen; hrsg. v. Graf C, Dordel S, Reinehr T; Deutscher Ärzte-Verlag GmbH, Köln 2007, 39-60

Emerson E (2009): Overweight and obesity in 3-5-year-old children wth and without developmental delay. Pub Health 123(2):130-133

Emerson E, Robertson J (2010): Obesity in young children with intellectual disabilities or borderline intellectual functioning. Int J Pediatr Obes. Epub Mar 9, 2010

Erb J, Winkler G (2003): Rolle der Nationalität bei Übergewicht und Adipositas bei Vorschulkindern. Monatsschr Kinderheilkd, 152:291-298

Farooqi S (2007): Insights from Genetics of Severe Childhood Obesity. Horm Res, $\underline{68: 5-7}$

Feig DI, Johnson RJ (2003): Hyperuricemia in childhood primary hypertension. Hypertension, 42:247-252

Fischer-Posovszky P, Wabisch M (2004): Entwicklung und Funktion des Fettgewebes. Monatsschr Kinderheilkd, 152:834-842

FKE- Forschungsinstitut für Kinderernährung Dortmund: Ernährungsepidemiologie. http://www.fke-do.de/content.php?seite=seiten/inhalt.php\&details=561 (Letzter Zugriff: 25.01.2009)

Ford ES, Li C (2008): Defining the Metabolic Syndrome in Children and Adolescents: Will the real Definition Please Stand Up? J Pediatr, 152(2):160-164

Fowler B (2005): Homozystein - ein unabhängiger Risikofaktor für kardiovaskuläre und thrombotische Erkrankungen. Ther Umsch, $\underline{62: 9}$ 
Franco MCP, Christofalo DMJ, Sawaya AL, Ajzen SA, Sesso R (2006): Effects of Low Birth Weight in 8- to 13-Year-Old Children, Implications in Endothelial Function and Uric Acid Levels. Hypertension, $\underline{48}: 45-50$

Gillman MW, Rifas-Shiman S, Berkey CS, Field AE, Colditz GA (2003): Maternal Gestational Diabetes, Birth Weight, and Adolescent Obesity. Pediatr, 111:e221-e226

Gösis- Göttinger Statistisches Informationssystem.

http://www.goesis.goettingen.de/themenfelder/unterthemen.php?navld=03\&uld=32\&maintopi $\mathrm{c}=2$ \&docname=020. (Letzter Zugriff: 09.02.2010)

Graf C, Dordel S:Sport und Bewegung bei Adipositas- Prävention und Therapie; in:

Adipositas in Praxi, Multimodale Konzepte für das Kindes- und Jugendalter; hrsg. v. Reinehr T, Wabisch M; Hans Marseille Verlag GmbH, München 2006, 43-53

Häger A, Sjöstrm L, Arvidsson B, Björntorp P, Smith U (1977): Body fat and adipose tissue cellularity in infants: a longitudinal study. Metabolism, 26(6):607-14.

Hahne D (2001): Frühgeburt durch pH-Selbstkontrolle vermeiden. Dtsch Ärztebl, 12:739740

Hampson SE, Andrews JA, Peterson M, Duncan SC (2007): A Cognitive-Behavioral Mechanism Leading to Adolescent Obesity: Children's Social Images and Physical Activity. Ann Behav Med, 34:287-294

Heins E, Seitz C, Schütz J, Toschke AM, Harth K, Letzel S, Böhler E (2007):

Schlafenszeiten, Fernseh- und Computergewohnheiten von Grundschulkindern in

Deutschland. Gesundheitswesen, $\underline{69}: 151-157$

Herold G: Innere Medizin; Dr. med. Gerd Herold, Köln 2007

Hinney B (2008): PCO-Syndrom (PCOS). http://wwwuser.gwdg.de/ ukfh/hormon/html/pcosyndrom.html

(Letzter Zugriff:20.06.2008)

Hofbauer KG (2002): Pathophysiologie der Adipositas: Moleküle, Mechanismen, Modelle. Schwei Med Forum, 40:937-944

levers-Landis CE, Redline S (2007): Pediatric Sleep Apnea. Am J Respir Crit Care Med, $\underline{175}: 436-441$

IDF (International Diabetes Federation) (2006): The IDF consensus worldwide definition of the metabolic Syndrome. http://www.idf.org/webdata/docs/MetS_def_update2006.pdf (Letzter Zugriff: 11.02.2010)

Infodienst Verbraucherschutz • Ernährung • Landwirtschaft e. V. (aid):

Lebensmittelpyramide. http://www.aid.de/ernaehrung/ernaehrungspyramide.php (Letzter Zugriff:19.04.2008)

IOTF (International Obesity Task Force) (2004): IOTF Childhood Obesity Report May 2004. www.iotf.org (Letzter Zugriff: 15.06.2005)

Janner M, Mullis PE, Flück Ch E (2006): Das metabolische Syndrom: Eine neue Kinderkrankheit?. Schweiz Rundsch Med Prax, 95:493-500 
Johnson RS, Feig DI, Herrera-Acosta J, Kang D-H (2005): Ressuraction of Uric as a Casual Risk Factor in Essential Hypertension. Hypertension, 45:18-20

Kaditis AG, Alexopoulus El, Hatzi F, Karadonta I, Chiadas K, Gourgoulianis K, Zintzaras E, Syrogiannopoulis GA (2008): Adiposity in relation to age as predictor of severity of sleep apnea in children with snorting. Sleep Breath, 12:25-31

Kelesidis T, Kelesidis I, Chou S, Mantzoros CS (2010): Narrative review: the role of leptin in human physiology: emerging clinical applications. Ann Intern Med, 152(2):93-100

Keller A, Klussek A, Gausche R, Hoepffner W, Kiess W, Keller E (2009): Gezielte primäre Adipositasprävention bei Kindern. Dtsch Med Wochenschr, 134:13-18

Keller KM (2004): Nicht alkoholische Fettleberkrankheit (NAFLK). Monatsschr Kinderheilkd, 152: 864-869

Kersting M: Ernährung; in: Adipositas in Praxi, Multimodale Konzepte für das Kindes- und Jugendalter; hrsg. v. Reinehr T, Wabisch M; Hans Marseille Verlag GmbH, München 2006, 27-41

Kleiser C, Schaffrath Rosario A, Mensink GBM, Prinz-Langenohl R, Kurth BM (2009):

Potential determinants of obesity among children and adolescents in Germany: results from the cross-sectional KiGGS study. BMC Pub Health, $\underline{9}: 46-60$

Koletzko B, Kries R, Closa R, Escribano J, Scaglioni S, Giovannini M,Beyer J, Demmelmair $\mathrm{H}$, Gruszfeld D, Dobrzanska A, Sengier A, Langhendries JP, Rolland Cachera MF, Grote V (2009) : Lower protein in infant formula is associated with lower weight up to age $2 \mathrm{y}$ : a randomized clinical trial. Am J Clin Nutr, 89:1836-45.

Kraft HG, Utermann G: Lipoprotein(a); in: Handbuch der Stoffwechselstörungen; hrsg. v. Schwandt P, Parhofer KG; Schattauer Verlag, Stuttgart 2007, 216-230

Kries R, Toschke AM, Koletzko B, Slikker W (2002): Maternat smoking during Pregnancy and Childhood Obesity. Am J Epidemiol, 156:954-961

Kries R, Toschke AM (2004): Perinatale Einflüsse auf das Adipositasrisiko. Monatsschr Kinderheilkd, 152:843-848

Kroke A, Hahn S, Buyken AE, Liese AD (2006): A comparative evaluation of two different approaches to estimating age at adiposity rebound. Int J Obes (Lond) $\underline{30: 261-266}$

Kromeyer-Hauschild K, Gläßler N, Zellner K (2008): Perzentile für den Taillenumfang von Jenaer Kindern im Alter von 6 bis 18 Jahren. Aktuel Ernährungsmed, 33:116-122

Krude H, Biebermann H, Schbabel D, Tansek MZ, Theunissen P, Mullis PE, Grüters A (2003): Obesity due to Proopiomelanocortin Deficiancy: Three New Cases and Treatment Trails with Thyroid Hormone and ACTH 4-10. J Clin Endocr Metab, 88:4633-4640

Kurth BM, Schaffrath Rosario A (2007): Die Verbreitung von Übergewicht und Adipositas bei Kindern und Jugendlichen in Deutschland. Bundesgesundheitsbl-GesundheitsforschGesundheitsschutz, 무:736-743

Lampert T, Mensink GBM, Romahn N, Woll A (2007 a): Körperlich-sportliche Aktivität von Kindern und Jugendlichen in Deutschland. Bundesgesundheitsbl-GesundheitsforschGesundheitsschutz, $\underline{\text { 00:634-642 }}$ 
Lampert T, Sygusch R, Schlack R (2007 b): Nutzung elektronischer Medien im Jugendalter. Bundesgesundheitsbl-Gesundheitsforsch-Gesundheitsschutz, 50:643-652

Lande MB, Carson NL, Roy J, Meagher CC (2006): Effects of Childhood Primary Hypertension on Carotid Intima Media Thickness, A Matched Controlled Study. Hypertension, $\underline{48}: 40-44$

Lange C, Schenk L, Bergmann R (2007): Verbreitung, Dauer und zeitlicher Trend des Stillens in Deutschland. Bundesgesundheitsbl-Gesundheitsforsch-Gesundheitsschutz, 50:624-633

Lange M, Kamptsiuris P, Lange C, Schaffrath Rosario A, Stolzenberg H, Lampert T (2007): Messung sozialdemographischer Merkmale im Kinder- und Jugendgesundheitssurvey (KiGGS) und ihre Bedeutung an der Einschätzung des allgemeinen Gesundheitszustandes. Bundesgesundheitsbl-Gesundheitsforsch-Gesundheitsschutz, 50:578-589

Lissau I, Sørensen TIA (1994): Parental neglect during childhood and increased risk of obesity in young adulthood. Lancet, $\underline{343: 324-327}$

Meinlschmidt G, Referat Gesundheitsberichterstattung, Epidemiologie, Gemeinsames Krebsregister, Sozialstatistisches Berichtswesen, Gesundheits- und Sozialinformationssysteme (2008): Gesundheitsberichterstattung Berlin Basisbericht 2006/2007 Daten des Gesundheits- und Sozialwesens.

Mensink GBM, Lampert T, Bergmann E (2005): Übergewicht und Adipositas in Deutschland 1984-2003. Bundesgesundheitsbl-Gesundheitsforsch-Gesundheitsschutz, 48:1348-1356

Mensink GBM, Kleiser C, Richter A (2007): Lebensmittelverzehr bei Kindern und Jugendlichen in Deutschland. Bundesgesundheitsbl-Gesundheitsforsch-Gesundheitsschutz, 50:609-623

Metcalf BS, Voss LD, Hosking J, Jeffrey AN, Wilkin (2008): TJ.Physical activity at the government-recommended level and obesity-related health outcomes: a longitudinal study Arch Dis Child. 93(9):772-7.

Nguyen M, Mitsnefes M (2007): Evaluation of hypertension by the general pediatrician. Curr Opin Pediatr, 19:165-169

NHBPEP (National High Blood Pressure Education Program Working Group on High Blood Pressure in Children and Adolescents, 2004): The Fourth Report on the Diagnosis, Evaluation, and Treatment of High Blood Pressure in Children and Adolescents. Pedeatrics, 114: $555-576$

Nixon GM, Thompson JM, Han DY, Becroft DM, Clark PM, Robinson E, Waldie KE, Wild CJ, Black PN, Mitchell EA (2008): Short sleep durationin middle childhood: riskfactors and consequences. Sleep, $\underline{31: 71-78}$

Nobili V, Manco M, Ciampalini P, Diciommo V, Devito R, Piemonte F, Comparcola D, Guidi $\mathrm{R}$, Marcellini $\mathrm{M}$ (2006): Leptin, free leptin index, insulin resistance and liver fibrosis in children with non-alcoholic fatty liver disease. Eur J Endocrin, 155:735-743

Olshansky SJ, Passaro DJ, Hershow RC, Layden J, Carnes BA, Brody J, Hayflick L, Butler RN, Allison DB, Ludwig DS (2005): A potential decline in life expectancy in the United States in the $21^{\text {st }}$ century. N Engl J Med, 352:1138-1145 
Ong KK (2007): Catch-up growth in small for gestational age babies: good or bad? Curr Opin Endocrinol Diabetes Obes, 14:30-34

Reinehr T (2005): Folgeerkrankungen der Adipositas im Kindes- und Jugendalter. http://www.a-g-a.de/aga_content.html (Letzter Zugriff: 20.01.2008)

Reinehr T, Wabisch M: Medizinische Ursachen der Adipositas und diagnostisches Vorgehen; in: Adipositas in Praxi, Multimodale Konzepte für das Kindes- und Jugendalter; hrsg. v. Reinehr T, Wabisch M; Hans Marseille Verlag GmbH, München 2006, 65-72

Reinehr T, Holl RW, Wabisch M (2008): The German Working Group of Obesity in Childhood and Adolescence (AGA): improving the qualitiy of care for overweight and obese children in Germany. Obes Facts, 1(1):26-32. Epub 2008 Feb 8

Reinehr T, Widhalm K, I'Allemand D, Wiegand S, Wabitsch M, Holl RW on behalf of the APVWiss Study Group (2009): Two-year follow-up in 21,784 overweight children and adolescents with lifestyle intervention. Obes (Silver Spring):17(6):1196-9. Epub 2009 Feb 19

Reinehr T, Dobe M, Winkel K, Schäfer A, Hoffmann D (2010): Obesity in Disabled Children and Adolescents. Dtsch Arztebl Int, 107(15):268-275

Röbl M, Lakomek M, Gahr M: Prävalenz chronischer Krankheiten bei Kindern und Jugendlichen; in: Zukunft sichern: Senkung der Zahl chronisch Kranker; hrsg. v. Schauder P, Berthold H, Eckel H, Ollenschläger G; Deutscher Ärzte-Verlag GmbH, Köln 2006, 87-100

Röbl M, Knerr I, Keller KM, Jaeschke R, Hoffmeister U, Reinehr T, Holl WR (2008): Adipositas bei Kindern und Jugendlichen und ihrer Eltern: Assoziation des standardisierten BMI zwischen Patienten, Eltern und Geschwistern anhand multizentrischer APV-Daten. Dtsch Med Wochenschr, 133: 2448-2453

Rolland-Cachera MF, Sempé M, Guilloud-Bataille M, Patois E, Péquignot-Guggenbuhl F, Fautrad V (1982): Adiposity indices in children. J Clin Nutr, 36:178-184

Saland JM (2007): Update on the metabolic syndrome in children. Curr Opin Pediatr, 19: 183-191

Sandiraz I (2010): Gesprächsnotiz der Verfasserin mit Iza Sandiraz dem Integrationsbeauftragten des Landkreises Göttingen vom 09.02.2010 in Göttingen

Schauder J, Arends J: Erfassung und Beurteilung des Ernährungszustandes; in: Ernährungsmedizin; hrsg. v. Schauder P, Ollenschläger G; Elsevier GmbH, München 2006, 477-497

Schenk L: KiGGS-Studie zur Gesundheit von Kindern und Jugendlichen in Deutschland, Migrantenbericht. http://www.kiggs.de/experten/downloads/dokumente/Migrantenbericht.pdf (Letzter Zugriff: 08.02.2010)

Schenk L, Ellert U, Neuhauser H (2007): Kinder und Jugendliche mit Migrationshintergrund in Deutschland. Bundesgesundheitsbl-Gesundheitsforsch-Gesundheitsschutz, 50:590-599

Schwimmer JB, Deutsch R, Kahen T, Lavine JE, Stanley C, Behling C (2009): Prevalence of fatty liver in children and adolescents. Pediatr, 118(4): 1388-1393 
Shine NP, Lannigan FJ, Coates HL, Wilson A (2006): Adenotonsillectomy for obstructive sleep apnea in obese children: effects on respiratory parameters and clinical outcome. Arch Otolaryngol Head Neck Surg, 132:1132-1137

Shrewsbury V, Wardle J (2008): Socioeconomic status and adiposity in childhood: a systematic rewiev of cross-sectional studies 1990-2005. Obesity (SilverSpring), 16(2): 275284.

Singh GK, Siahpush M, Kogan MD (2010): Risinig social inequalities in US childhood obesity. Ann Epidemiol, 20(1):40-52

Sitzmann FC: Duale Reihe Pädiatrie, 3. Auflage; Georg Thieme Verlag KG, Stuttgart 2007

Soultan Z, Wadowski S, Rao M, Kravath RE (1999): Effect of treating obstructive sleep apnea by tonsillectomy and/or adenoidectomy on obesity in children. Arch Pediatr Adolesc Med, 153:33-37

Stanger O, Herrmann W, Pietrzik K, Fowler B, Geisel J, Dierkes J, Weger M on behalf of the DACH-LIGA Homozystein e.V. (2003): DACH-LIGA Homozystein (German, Austrian and Swiss Homozystein Sociaty): Consensus Paper on the Rational Clinical Use of Homozysteine, Folic Acid, B-Vitamines in Cardiovascular an Thrombotic Diseases: Guidelines and Recommendations. Clin Chem Lab, 41:1392-1403

Statistisches Bundesamt (2008): Familienland Deutschland, Begleitmaterial zur Pressekonferenz am 22.Juli 2008 in Berlin.

http://www.destatis.de/jetspeed/portal/cms/Sites/destatis/Internet/DE/Presse/pk/2008/Familie nland/Pressebroschuere__Familienland,property=file.pdf (Letzter Zugriff: 08.02.2010)

Stolzenberg H, Kahl H, Bergmann KE (2007): Körpermaße bei Kindern und Jugendlichen in Deutschland. Bundesgesundheitsbl-Gesundheitsforsch-Gesundheitsschutz, 50:659-669

Stunkard AJ, Harris JR, Pedersen NL, McClearn GE (1990): The body-mass index of twins who have been reared apart. N Engl J Med. 322(21):1483-7.

Taylor RW, Grant AM, Goulding A, Williams SM (2005): Early adiposity rebound: review of papers linking the subsequnt obesity in children and adults. Curr Opin Clin Nutr Metab Care, 8:607-612

Urbina E, Alpert B, Flynn J, Hayman L, Harshfield GA, Jacobson M, Mahoney L, McCrindle B, Mietus-Snyder M, Steinberger J, Daniels S (2008): Ambulatory Monitoring in Children and Adolescents: Recommendation for Standard Assessment- A Scientific Statement From the American Heart Association.

Hypertension, 52: 433-451

Utermann G (1989): The mysteries of lipoprotein(a). Science, 246:904-910

Wabisch M: Diagnostik medizinischer Folgeerkrankungen; in: Adipositas in Praxi, Multimodale Konzepte für das Kindes- und Jugendalter; hrsg. v. Reinehr T, Wabisch M; Hans Marseille Verlag GmbH, München 2006, 85-97

Warschburger P: Psychologisch-psychiartrische Grund- und Folgeerkrankungen; in: Adipositas in Praxi, Multimodale Konzepte für das Kindes- und Jugendalter; hrsg. v. Reinehr T, Wabisch M; Hans Marseille Verlag GmbH, München 2006, 73-84 
Watkins MG, Clark KM, Foster CM, Welch KB, Kasa-Vubu JZ (2007): Realtionships among Body Mass Index, Parenteral Perceptions, Birthweight and Parenteral Weight after Referral to a Weight Clinic. J Natl Med Assoc, 99:908-913

Wertheim K, Sobczynska-Tomaszewksa A, Bal J (2007): Search fort he ethiopathogenesis of polycystic ovary syndrome (PCOS). Ginekol Pol, $\underline{78: 626-631}$

Wiegand S, Keller K-M, Röbl M, l'Allemand D, Reinehr T, Widhalm K, Holl RW (2010): Obese Boys at increased risk for Non-Alcoholic Liver Disease. Evaluation of 16390 Overweight or Obese Children and Adolescents. Int J Obes (Lond). 2010 Jun 8 [Epub ahead of print]

Williams SM (2005): Weight and height growth rate and the timing of adiposity rebound. Obes Res, 13:1123-1130

Williams SM, Goulding A (2008): Patterns of Growth Associated With the Timing of Adiposity Rebound. Obesity (Silver Spring) Dec 4, 2008 (Epub ahead of print)

Wills M (2004): Orthopedic complications of childhood obesity. Pediatr Phys Ther, 16: 230235

Witkowski R, Prokop O, Ullrich E: Lexikon der Syndrome und Fehlbildungen, 6. Auflage; Springer-Verlag Berlin Heidelberg 1999 


\section{Danksagung}

Mein Dank gilt vor allem meinem Betreuer Dr. med. Markus Röbl und meiner Doktormutter Frau Prof. Dr. med. Jutta Gärtner, auf deren Anregung ich diese Doktorarbeit erstellt habe, für die investierte Zeit.

Weiterhin danke ich allen, die mich im langen Prozess der Entstehung dieser Arbeit auf vielfältige Weise unterstützt und ermutigt haben, insbesondere Frank Konietschke, Sven Ricke, Egon Zimmermann, Andreas Schlemminger und meinen Eltern, die mir dieses Studium ermöglicht haben. 


\section{Ausführlicher Lebenslauf}

Mein Name ist Tatjana Tamara König. Ich wurde am 09.12.1983 in Hannover geboren.

Meine Schulzeit verbrachte ich in Seesen am Harz, wo ich zwischen 1990 und 2003 die Grundschule Jahnstraße, die Orientierungsstufe und schließlich das Jacobson Gymnasium besuchte. In der elften Klasse von August 2000 bis Juni 2001 verbrachte ich ein High School Jahr in Texas bei einer Gastfamilie, wo ich zuerst Schülerin der Cayuga High School war, bevor ich im November ein Stipendium für die All Saints Episcopal High School in Tyler erhielt. Nach meiner Rückkehr setzte ich meine Ausbildung im zwölften Jahrgang am Jacobson Gymnasium Seesen fort, wo ich 2003 die allgemeine Hochschulreife mit der Note 1,5 erlangte. Während dieser Zeit arbeitete ich aushilfsweise nach der Schule und an den Wochenenden bei der Video Buster Dienstleistungsgesellschaft $\mathrm{mbH} /$ Grebenstein Holding in Seesen, wo Büro- und Logistikarbeiten, Kundenbetreuung und Dolmetscherdienste für Englisch zu meinen Aufgaben gehörten.

Nach der Schule entschied ich mich für das Studium der Medizin an der Georg-AugustUniversität Göttingen, das ich im September 2003 begann. In diesem Rahmen absolvierte ich neben den Pflichtpraktika Famulaturen in den Bereichen Anästhesie, Gynäkologie und Kinderheilkunde. Während meiner Studienzeit arbeitete ich als wissenschaftliche Hilfskraft im Sozialpädiatrischen Zentrum Göttingen. Das praktische Jahr absolvierte ich von August 2008 bis Juli 2009 in der Abteilung für Gastroenterologie der Universitätsmedizin Göttingen, der Klinik für Allgemeinchirurgie des Pius Hospital und der Klinik für Unfallchirurgie des evangelischen Krankenhauses in Oldenburg, der Klinik für Pädiatrie des Nepean Hospital in Sydney, Australien und der Kinderklinik des St. Vinzenz Hospital in Paderborn. Für meinen Auslandsaufenthalt während des PJ erhielt ich ein Stipendium von „Stethosglobe.de“. Am 04.12.2009 schloss ich mein Medizinstudium an der Georg-August-Universität Göttingen mit der Note gut $(2,16)$ ab und erlangte die Approbation im Januar 2010.

Am ersten März 2010 trat ich meine derzeitige Arbeitsstelle als Assistenzärztin in der Klinik für Kinderchirurgie der Universitätsmedizin Mainz an. Seither lebe ich in Mainz mit dem Ziel, Fachärztin für Kinderchirurgie zu werden. 